Association of Nucleoside Diphosphate Kinase with Microtubule-Based Structures

Kimberly Ann Parrott Mitchell

Forest, Virginia

MS, University of Virginia, 2006

BS, Liberty University, 1999

A Dissertation presented to the Graduate Faculty

of the University of Virginia in Candidacy for the Degree of

Doctor of Philosophy

Department of Molecular Physiology and Biological Physics

University of Virginia

August, 2008

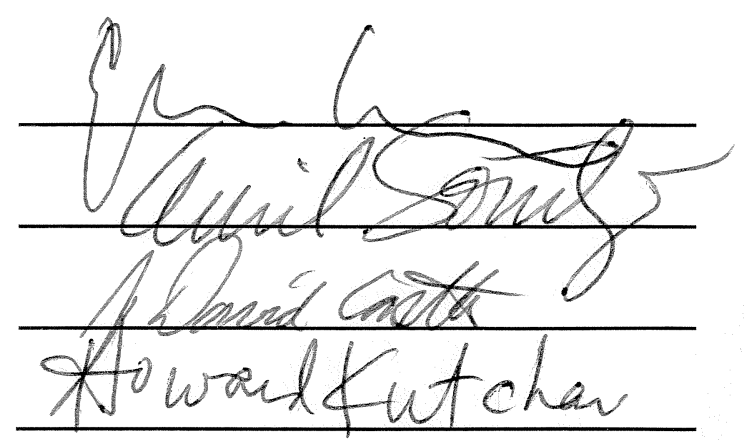




\section{DEDICATION}

To Jame, who makes life more enjoyable than I ever imagined it could be.

Thank you for being my partner in life. 


\section{ACKNOWLEDGEMENTS}

Many people contributed to this work in various ways. My parents, Steve and Shirley Parrott, have been my supporters throughout my life, encouraging me to do my best and pursue an education. My sister Jen has kept my spirits up through her humor, and my brother Mike has always provided a listening ear. Grandma has been ever proud of me and available to talk, even into wee hours of the morning if necessary to keep me awake while driving home.

Several of my undergraduate professors have shown continued interest in my progress, especially Dr. Norm Reichenbach, Dr. David DeWitt, and my undergraduate advisor, Dr. Charles Detwiler, who has remained an advisor and friend throughout the years. His wisdom and insight are much appreciated.

Some students are fortunate to have a good graduate advisor. I, however, have been blessed with two excellent advisors: Dr. Gabor Szabo and Dr. Angelo Otero. Both have been supportive beyond words. They have helped me grow from a technician, to a graduate student, to a professor. They have mentored me, befriended me, and taught me many things, including to think critically and to write well. Past members of their labs also assisted me, including Dr. Joseph Akar, Dr. Betty Gallagher, Dr. Attila Szabo and Ms. Wanxia Wang.

My committee members have been supportive by allowing me to use their labs and giving me helpful advice and constructive criticism throughout my graduate career. Dr. David Castle allowed my to do a rotation in his lab, and to use microscopy equipment for countless hours. The whole Castle lab has been helpful throughout the years. Dr. Howard Kutchai gave valuable advice about graduate school in general and made his lab 
equipment available to me on a regular basis. Dr. Avril Somlyo also provided expertise and use of equipment. The entire Somlyo lab has been helpful, especially Mr. Joe Craft, who used his mastery of the electron microscope to provide the EM pictures of primary cilia, and Dr. Ruoya Ho who skillfully took pictures of the microtubule-associated vesicles with both electron and confocal microscopes.

I am indebted to Dr. Peter Horanyi for spending considerable time helping with the in silico docking images and teaching me to use PyMol.

Ms. Kristi Foell and Mrs. Terry Hall assisted in keeping my house clean while I was working and Terry also provided a critical, non-scientific eye in proofreading both my most recent paper for publication and this dissertation.

Many other friends have done exactly what friends are meant to do, and I do not have room to list them all here. The Ross/Haskew/Smith clan has provided constant moral support and pleasant hours of distraction. Some friends have helped me directly in this work by allowing me to stay at their house on a moment's notice when my experiments went late: Christina Haden, Melissa Hatley, Liz Lawhorne, Alison Sivak, and Joanne Scanlon. Having Joanne in the lab next door was a blessing. She made this journey much more enjoyable, and I am glad to have shared the last eight years in Jordan hall with her. Many other members of the Nakamoto lab, past and present, have been helpful as well. Dr. Robert Nakamoto assisted with graduate school in general and allowed me to use his lab several times, especially for a rotation in which I learned much molecular biology.

Lisa Geddis is one of the most knowledgeable people I know, and was willing to share that knowledge with me when needed. She is an expert in the lab and of 
Charlottesville in general and has been an invaluable resource as well as a friend. I am indebted to her and to the Tamm lab was for allowing me to use their nitrogen on a regular basis.

Carol Yowell and Cathy Black helped immensely with orders and anything else that was needed; I am grateful for their advice and friendship. Pam Mullinex assisted in making sure all my official forms were in on time, John Chapman gave technical support, and Ann Folsom, Kathleen Foster, Tracy Gardner, and Howard Phipps always lent support when needed.

I thank Dr. Ioan Lascu and Dr. Marie-Lise Lacombe for generously sharing constructs and antibodies, and Dr. Nancy K. Willis (University of Texas Medical Branch) for invaluable advice on the culture of A6 cells.

Monoclonal antibodies developed by T. August (1D4B), J. Scholey (SUK4) and D. Fambrough (a5) were obtained from the Developmental Studies Hybridoma Bank eveloped under the auspices of the NICHD and maintained by The University of Iowa, Department of Biological Sciences (Iowa City, IA 52242).

Funding from both the NIH and the Cardiovascular Research Training Grant is much appreciated, as is the support of my graduate program, Molecular Medicine, and my department, Molecular Physiology and Biological Physics.

Most of all I thank my husband, Jamie Mitchell, who is my perfect match. He has loved me, supported me, helped me grow as a person and made me feel like a queen while doing so. He makes life enjoyable and more than any other person enabled me to achieve this goal. 
Table of Contents

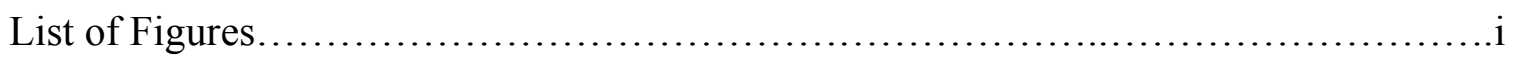

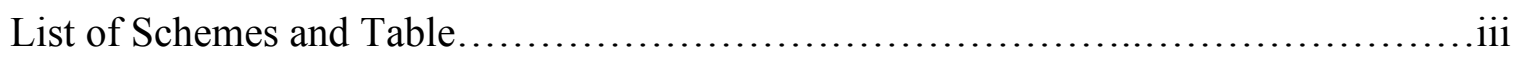

List of Abbreviations.........................................................

CHAPTER 1: Abstract and Introduction

1.1 Abstract.....................................................................

1.2 Introduction: Nucleoside Diphosphate Kinase (NDPK)

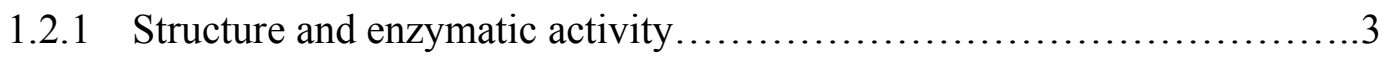

1.2.2 Additional functions of NDPK

1.2.2.1 NM23 and metastasis..................................... 7

1.2.2.2 DNA binding and transcription regulation....................8

1.2.2.3 Intracellular traffic .....................................8

1.2.2.4 Signal transduction...................................... 9

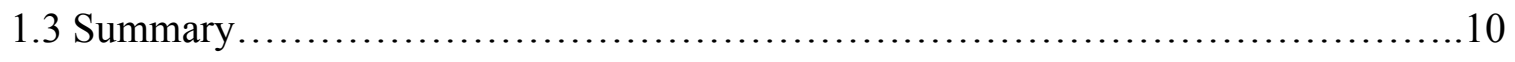

CHAPTER 2: Experimental Methods

2.1 Cell culture and transfection........................................... 11

2.2 Microscopy methods

2.2.1 Indirect immunofluorescence.................................... 12

2.2.2 Electron microscopy ........................................... 14 
2.3. Biochemical methods

2.3.1 SDS-PAGE and immunoblotting................................. 14

2.3.2. NDPK enzyme assay........................................ 15

2.4. Isolation of subcellular fractions

2.4.1. Isolation of microtubule-associated endocytic vesicles

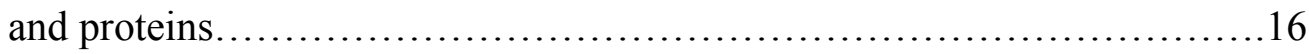

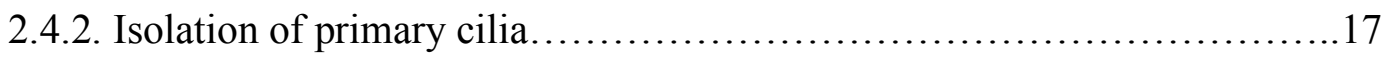

2.5 Protein purification

2.5.1 Purification of NDPK from human erythrocytes.......................20

2.5.2 Bacterial expression and purification of human NDPK A................22

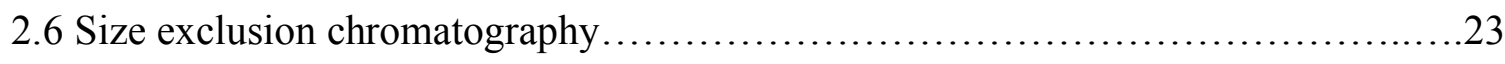

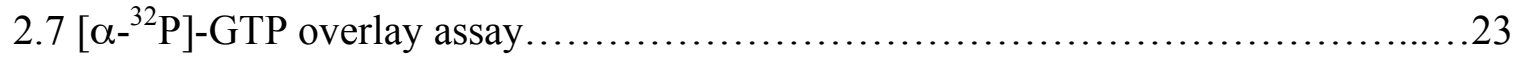

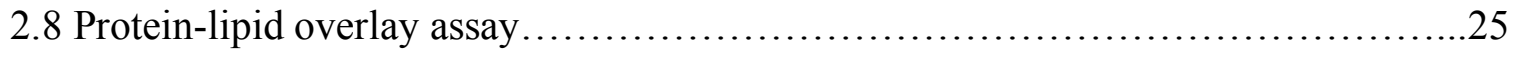

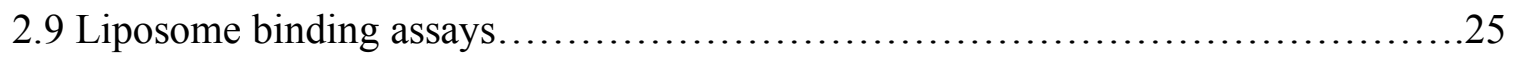

CHAPTER 3: NDPK Localizes to Vesicles Associated with Microtubules

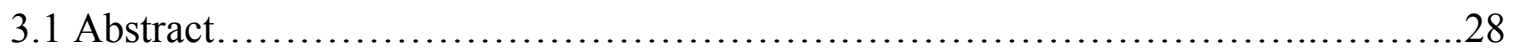

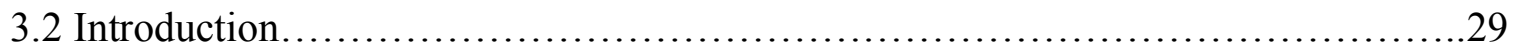

3.3 Results

3.3.1 NDPK is located in microtubule-associated vesicles and this interaction requires an intact microtubule network $\ldots \ldots \ldots \ldots \ldots \ldots \ldots \ldots 31$

3.3.2 NDPK binds to membranes associated with microtubules .................33 
3.3.3 NDPK localizes to the majority of vesicular structures associated with microtubules........................................ 36

3.3.4 Nucleotides release NDPK from microtubule-bound vesicles ..............38

3.3.5 Characterization of the process leading to NDPK dissociation from microtubule-associated vesicles......................40

3.3.6 The binding of NDPK to vesicles is controlled by its catalytic cycle ......44

3.3.7 NDPK binds directly to phospholipids..............................50

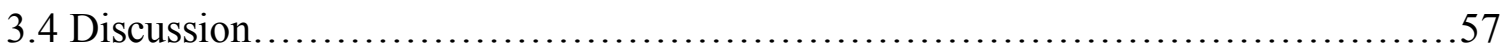

CHAPTER 4: NDPK Moves into the Developing Primary Cilium

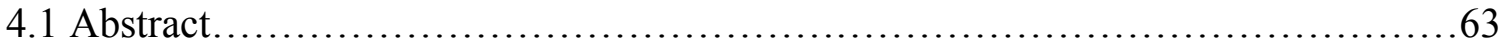

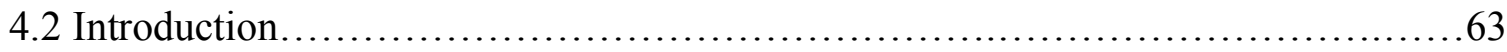

4.3 Results

4.3.1 NDPK is present in primary cilia of NIH-3T3 fibroblasts...............66

4.3.2 NDPK localizes to primary cilia of A6 kidney epithelial cells............67

4.3.3 NDPK distribution during primary cilium development in A6 cells........71

4.3.4 NDPK is present in isolated primary cilia...........................68

4.3.5 NDPK is present in the matrix and membranes of

primary cilia isolated from A6 cells................................. 77

4.3.6 Appearance of isolated primary cilia............................. 78

4.3.7 Expression of EGFP-tagged NDPK. ............................ 80

4.3.8 Binding partners of NDPK and another NM23/NDPK are present in primary cilia....................................... 80 


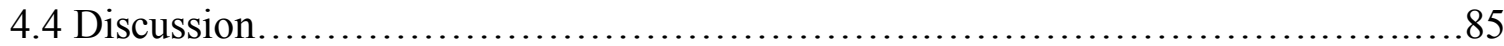

CHAPTER 5: Conclusion and References

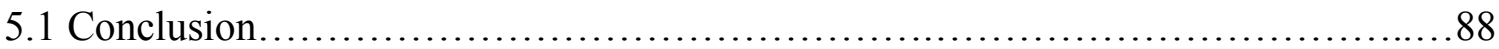

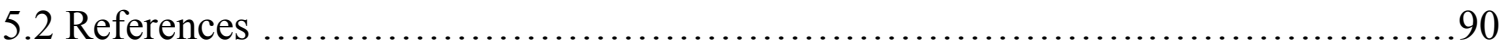

APPENDIX A: Background Data............................................... 113 


\section{List of Figures}

Figure 1. Structure of human NDPK B hexamer.................................

Figure 2. Purification of NDPK from human erythrocytes..........................21

Figure 3. Purification of human NDPK A.................................... 24

Figure 4. Localization of NDPK to microtubular-associated vesicles..................32

Figure 5. Size distribution of NDP kinase-labeled vesicles in NIH-3T3 cells............35

Figure 6. GTP releases NDPK from taxol-stabilized microtubules and associated vesicles.................................................. 35

Figure 7. NDPK localizes to most vesicular structures associated with

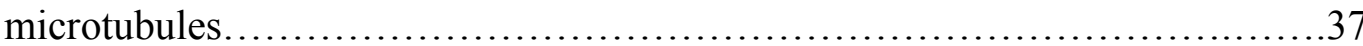

Figure 8. Size distribution of microtubule-associated vesicles isolated from NIH-3T3 cells .39

Figure 9. Both ATP and GTP release NDPK from taxol-stabilized microtubules and associated vesicles.

Figure 10. NDPK can re-bind to GTP-stripped pellets, and re-binding is saturable.....41

Figure 11. NDPK is released from microtubule-associated vesicles by GTP...........43

Figure 12. NDPK A and B are present in microtuble-associated vesicles, but NDPK $\mathrm{C}$ is not

Figure 13. Small GTP binding proteins are not released from microtubule-associated vesicles by GTP .45

Figure 14. The interaction of NDPK with membranes is controlled by its substrates, and is abolished by ATP depletion. 
Figure 15. NDPK binds directly to phospholipids.................................5

Figure 16. Interaction of NDPK from cytosol with liposomes of defined

composition

Figure 17. Interaction of purified NDPK from human erythrocytes with

liposomes of defined composition......................................55

Figure 18. Interaction of cytosolic NDPK with liposomes containing

phospholipids, cholesterol and sphingomyelin............................56

Figure 19. Space-filled model of NDPK with ADP bound to active site................60

Figure 20. In silico docking of phosphatidic acid into the nucleotide binding site of an NDPK monomer..........................................60

Figure 21. NDPK localizes to the primary cilium in NIH-3T3 fibroblasts..............68

Figure 22. Localization of NDPK in primary cilia of A6 kidney epithelial cells..........70

Figure 23. NDPK stains primary cilia in a punctate pattern..........................72

Figure 24. Localization of NDPK during primary cilium development. .................72

Figure 25. Translocation of NDPK into the primary cilium is length-dependent..........74

Figure 26. Cells remain intact after removal of primary cilia...................... 74

Figure 27. Biochemical characterization of isolated primary cilia. ....................76

Figure 28 . The primary cilia fraction contains whole cilia and ciliary tips. ..............79

Figure 29. Model of EGFP-tagged NDPK with an EGFP tag on each monomer........81

Figure 30. EGFP-tagged NDPK does not enter the primary cilium of NIH-3T3 cells...82

Figure 31. EGFP-tagged NDPK does not enter the primary cilium of A6 cells..........82

Figure 32 . Binding partners of NDPK localize to primary cilia.................... 84

Figure 33. NM23-H5 localizes to some, but not all, primary cilia....................84 


\section{List of Schemes and Table}

\section{Schemes}

Scheme 1. Isolation of microtubule-associated vesicles.............................. 18

Scheme 2. Determination of NDPK's location in microtubule-associated vesicles.......18

Scheme 3. NDPK-catalyzed phosphate transfer in the presence of phospholipids

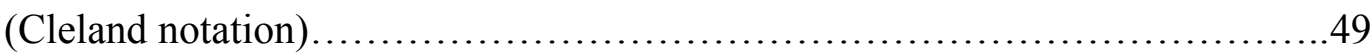

Table

Table 1. Lipid mixes used for liposome preparation............................26 


\section{List of Abbreviations}

\begin{tabular}{|c|c|}
\hline $5-\mathrm{HT}_{6}$ & serotonin \\
\hline Á & Angstrom \\
\hline $\mathrm{ADP}$ & adenosine diphosphate \\
\hline ATP & adenosine triphosphate \\
\hline Awd & abnormal wing discs \\
\hline $\mathrm{BCA}$ & bicinchoninic acid \\
\hline BSA & bovine serum albumin \\
\hline $\mathrm{CDP}$ & cytosine diphosphate \\
\hline $\mathrm{CK}$ & creatine kinase \\
\hline CMV & cytomegalovirus \\
\hline COPII & cytosolic coat protein complex II \\
\hline CTP & cytosine triphosphate \\
\hline $\mathrm{D} / \mathrm{A}$ & 2-deoxyglucose/sodium azide \\
\hline DIC & differential interference contrast \\
\hline TDP & thymidine diphosphate \\
\hline TTP & thymidine triphosphate \\
\hline DTT & 1,4-dithiothreitol \\
\hline EGFP & enhanced green fluorescent protein \\
\hline eNDPK & NDPK purified from human erythrocytes \\
\hline ER & endoplasmic reticulum \\
\hline $\mathrm{ER} \beta$ & estrogen receptor- $\beta$ \\
\hline
\end{tabular}




$\begin{array}{ll}\text { FGF } & \text { fibroblast growth factor } \\ \text { GDP } & \text { guanosine diphosphate } \\ \text { GDP } \beta \text { S } & \text { guanosine-5'-O-(2-thio)diphosphate } \\ \text { GIRK } & \text { G protein-coupled inward rectifier potassium channel } \\ \text { GMP-PNP } & \text { guanylyl-imidodiphosphate } \\ \text { GPCR } & \text { G protein-coupled receptor } \\ \text { GTP } & \text { guanosine triphosphate } \\ \text { GTP } \gamma \text { S } & \text { guanosine-5'-O-(3- thio)triphosphate } \\ \text { Hh } & \text { hedgehog } \\ \text { ICAP } & \text { integrin cytoplasmic domain associated protein } \\ \text { IgG } & \text { immunoglobulin G } \\ \text { IFT } & \text { intraflagellar transport } \\ \text { IPTG } & \text { isopropyl-beta-D-thiogalactopyranoside } \\ \text { LB } & \text { Luria broth } \\ \text { LDH } & \text { lactate dehydrogenase } \\ \text { MDCK } & \text { Madin-Darby canine kidney } \\ \text { MOPS } & \text { 3-(N-morpholino)propanesulfonic acid } \\ \text { Mt/Ves } & \text { isolated microtubule-associated vesicles } \\ \text { NDP } & \text { nucleoside diphosphate } \\ \text { NDPK } & \text { nucleoside diphosphate kinase } \\ \text { NM23 } & \text { nucleoside diphosphate kinase (non-metastatic) } \\ \text { NTP } & \text { nucleoside triphosphate } \\ \text { rNDPK } & \text { recombinant human NDPK A } \\ \text { Ho }\end{array}$




\begin{tabular}{|c|c|}
\hline OD & optical density \\
\hline PA & phosphatidic acid \\
\hline PBS & phosphate-buffered saline \\
\hline $\mathrm{PC}$ & Phosphatidylcholine \\
\hline PDGF & platelet-derived growth factor \\
\hline PE & phosphatidylethanolamine \\
\hline PEBP & phosphatidylethanolamine-binding protein \\
\hline PEP & phospho(enol)pyruvate \\
\hline PI & phosphatidylinositol \\
\hline PK & pyruvate kinase \\
\hline POMC & pro-opiomelanocortin \\
\hline PS & phosphatidylserine \\
\hline PTdIns & phosphatidylinositol \\
\hline Pvr & platelet-derived growth factor/vascular endothelial growth factor receptor \\
\hline $\mathrm{RB}$ & resuspension buffer \\
\hline RTK & receptor tyrosine kinase \\
\hline SDS-PAGE & sodium dodecyl sulfate polyacrylamide gel electrophoresis \\
\hline sst3 & somatostatin \\
\hline STRAP & serine/threonine receptor associated protein \\
\hline TBST & tris buffered saline with Tween-20 \\
\hline TGF $\beta$ & transforming growth factor- $\beta$ \\
\hline $\mathrm{TP} \beta$ & thromboxane A2 receptor \\
\hline TCA & trichloroacetic acid \\
\hline
\end{tabular}


UTP uridine triphosphate

VIM vimentin 


\section{CHAPTER 1: Abstract and Introduction}

\subsection{Abstract}

Cytosolic nucleoside diphosphate kinases (NDPKs) have been implicated in a variety of signaling pathways that occur at membranes, including those that control cell migration and spreading. This is particularly intriguing, as cytosolic NDPKs (NDPK A and NDPK B) are soluble proteins and do not have membrane-binding motifs, leading to the question: how do NDPK's participate in such a wide array of membrane signaling processes? Our lab has shown that one portion of cytosolic NDPK is translocated to the ruffling plasma membrane upon activation of both receptor tyrosine kinases (RTKs) and G protein-coupled receptors (GPCRs) and that the Rac1 signaling pathway is responsible for that migration. Although NDPK does not bind directly to Rac1, it moves to the cell periphery in conjunction with Rac1.

While investigating the association of cytosolic NDPK with the plasma membrane, we found that another pool of NDPK is bound to membrane vesicles that are associated with microtubules (Mt/Ves). A detailed study of this NDPK population shows that, unlike the pool that is involved in Racl signaling, NDPK's presence in these vesicles is not dependent on extracellular stimulation; rather, it is controlled by the nucleotide triphosphate to nucleotide diphosphate ratio ([NTP]/[NDP]), as evidenced by the effect of nucleotides on Mt/Ves isolated from fibroblasts. More importantly, purified and cytosolic NDPKs bind to both immobilized lipids and liposomes in a nucleotidesensitive manner. This indicates that NDPK can bind directly to intracellular membrane compartments, most likely to provide CTP for phospholipid biosynthesis and GTP for the many small GTPases involved in microtubule-dependent traffic. 
We also found that NDPK localizes to yet another microtubule-based cell compartment: the sensory primary cilium, an organelle implicated in many signaling pathways. NDPK enters the cilium during its development, when it reaches about 5.5 microns (or $24 \%$ of final primary cilia length) in A6 cells. In primary cilia NDPK is present in the soluble portion, or matrix, and in association with the membrane fraction. The function of NDPK within primary cilia is most likely to regenerate GTP for microtubule turnover and for signaling systems, making it an important contributor to primary cilia structure and function. 


\subsection{Introduction: Nucleoside Diphosphate Kinase (NDPK)}

\subsubsection{Structure and enzymatic activity}

Nucleoside diphosphate (NDP) kinases are ubiquitous enzymes that catalyze the rapid and reversible phosphorylation of nucleoside diphosphates (NDPs) by nucleoside triphosphates (NTPs), via a ping-pong mechanism involving a phosphohistidine intermediate, as schematized below:

$$
\begin{aligned}
& \mathrm{N}_{1} \mathrm{TP}+\text { E-His } \Leftrightarrow \mathrm{N}_{1} \mathrm{DP}+\text { E-His-P (1a) } \\
& \text { E-His-P }+\mathrm{N}_{2} \mathrm{DP} \Leftrightarrow \text { E-His }+\mathrm{N}_{2} \mathrm{TP}(1 \mathrm{~b})
\end{aligned}
$$

During the reaction, which has been well studied and documented (reviewed in Lascu and Gonin, 2000), NDPK is transiently phosphorylated on a histidine residue located in a small cavity. The properties of the resulting covalent intermediate have been thoroughly investigated (Lascu and Gonin, 2000). NDPKs are very efficient enzymes, with a turnover number on the order of $1000 / \mathrm{s}$, so each of the two steps is complete in less than 1 ms. Although NDPKs show preference for certain nucleotides, with GTP being favored by eukaryotic enzymes, they are not specific: the phosphate donor, $\mathrm{N}_{1} \mathrm{TP}$, and the acceptor, $\mathrm{N}_{2} \mathrm{DP}$, can be any of the nucleotides or deoxynucleotides found in cells, with ATP as the most likely donor because of its abundance. This lack of specificity is attributable to the absence of polar interactions between the protein and the base moiety of the nucleotide, which is largely exposed to the solvent. This is in contrast with the multiple interactions between the protein and the sugar and phosphate portions, which are buried in the active site (reviewed in Janin and Deville-Bonne, 2000). 
NDPK activity is very high in most organisms, where it catalyzes the final step of the synthesis of nucleotides such as GTP, UTP and CTP. Thus, NDPKs play an important role in supplying the nucleotides essential for the metabolism of nucleic acids, lipids, proteins and sugars (Lacombe et al., 2000).

NDPKs are well conserved and have been found in almost all organisms, from bacteria to plants and mammals, excluding only mycoplasma. To date, there have been nine human $n m 23$ genes identified (nm23-H1 to $H 9$; for review see Lacombe et al., 2000). The products of the first four genes have NDPK activity, and are highly homologous polypeptides composed of 152-187 amino acids. Studies of the 3D structure of these four NDPKs show that all form hexamers (Fig. 1). This is also true for most NDPKs including enzymes from insects and slime mold, with the exception of some bacterial enzymes, which are tetramers (Janin et al., 2000; Moynie et al., 2007). Hexamers isolated from mammalian cells are often of mixed composition (Gilles et al., 1991), containing both NDPK A (also referred to as NM23-H1 in humans, see below) and NDPK B (NM23-H2), the two predominant isoforms in mammalian cells. NDPKs A and B are primarily cytosolic although both isoforms sometimes localize to the nucleus, and are found in all cells, while other members of the NDPK family are specific components of certain tissues or organelles. NDPK C (Dr-NM23) is expressed at much lower levels, mostly in liver and kidney, and has a hydrophobic $\mathrm{N}$ terminus extension that may serve as a membrane anchor; in contrast, NM23-H4 and NM23-H6, both mitochondrial, are widely distributed. NM23-H5 protein is expressed in the flagella of spermatids and spermatozoa, where it associates with axoneme microtubules (Munier et 


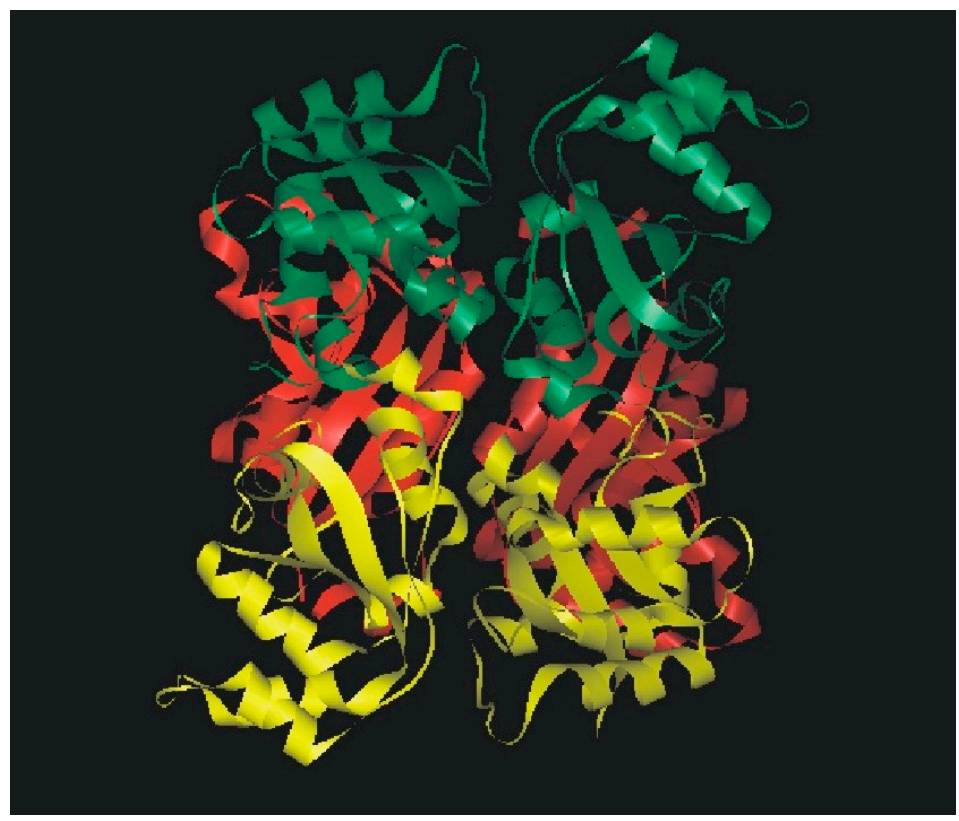

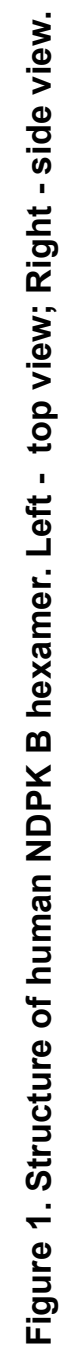


al., 2003). NM23-H7, NM23-H8 and NM23-H9 are expressed in the testis and respiratory tract, and contain more than one NDPK domain. NM23-H7 consists of an N-terminal DM10 domain and two NDPK modules. The function of the DM10 domain, which also occurs in multiple copies in other proteins, is unknown. NM23-H8 (or Sptrx-2; MirandaVizuete et al., 2003) is a fusion protein of an N-terminal thioredoxin domain followed by three NDPK domains, the first of which is truncated and the last two complete. NM23-H9 (or Txl-2; Sadek et al., 2003) consists of an N-terminal thioredoxin domain and a Cterminal NDPK domain. With the exception of H6, isoforms H5 to H9 have not been shown to have true NDPK activity (Lacombe et al., 2000).

Aside from the rephosphorylation of nucleoside diphosphates, NDPK has been reported to have many other functions. It reportedly phosphorylates substrates as diverse as proteins (Engel et al., 1995; Wagner and Vu, 1995; Freije et al., 1997; Wagner et al., 1997; Wagner and Vu, 2000; Hartsough et al., 2002; Besant and Attwood, 2005, Srivastava et al., 2006) and geranyl and farnesyl pyrophosphates (Wagner and $\mathrm{Vu}, 2000$ ). However, these non-NDPK activities are slow (measured in $\mathrm{nmol} / \mathrm{mg} / \mathrm{min}$, as opposed to $\mu \mathrm{mol} / \mathrm{mg} / \mathrm{min}$ ) and most are stoichiometric, rather than catalytic. Additionally, many of these experiments were carried out under non-physiological conditions. Indeed, the majority of these results have eventually been shown to be artifactual, arising from contaminating ADP, or to be non-enzymatic reactions that happen after the proteins are denatured (Bominaar et al., 1994; Levit et al., 2002). Similar artifacts are found in a number of studies that purport direct phosphorylation of the GDP bound to G proteins by NDPK (reviewed in Otero, 2000). 
Members of the NDPK/NM23 family of proteins are reportedly involved in growth, differentiation and development. Thus, loss of the Drosophila homolog of NDPK causes imaginal disc defects, leading to its name Awd (abnormal wing discs) (Dearolf et al., 1988a, 1988b; reviewed in Timmons and Shearn, 2000). Awd is also involved in the mobilization and migration of epithelial cells that take place during normal embryonic development. Several studies have implicated NDPKs in cell differentiation and development of mammalian cells as well. For instance, mammary gland development is impaired in NDPK A knockout mice, and induction of differentiation in glioma cells results in increased expression of NDPK A (reviewed in Lombardi, 2006). The biochemical mechanisms proposed to explain these diverse functions range from the DNA binding capability of NDPK to a role in transmembrane signaling; these novel roles of NDPK are briefly reviewed below.

\subsubsection{Additional functions of NDPK}

\subsubsection{NM23 and metastasis}

NDPK was originally cloned from Dictyostelium discoideum (Lacombe et al., 1990). The sequence was found to be identical to that of a $23-\mathrm{kDa}$ protein, NM23, which was discovered in $\boldsymbol{n}$ on-metastatic murine melanoma cells (Steeg et al., 1988) and found to be expressed at reduced levels in some metastatic cancers (for reviews, see Hartsough and Steeg, 2000; Roymans et al., 2002; Steeg, 2004; Boisson et al., 2005; Lombardi, 2006; and Steeg and Theodorescu, 2008). Expression of NDPK A and NDPK B are both reduced in these metastatic tumors, but NDPK A is reduced to a larger extent, implying that the expression of the two isoforms is regulated by distinct mechanisms (Stahl et al., 
1991). Metastasis suppression by NDPK is reportedly not dependent on the enzyme's NDPK activity (MacDonald et al., 1993; Kim et al., 2003).

\subsubsection{DNA binding and transcription regulation}

NDPKs later proved to have cellular functions beyond nucleotide regeneration and metastasis suppression. Many members of this family bind DNA and can act as transcription factors; for instance, NDPK A and NDPK B bind to the promoter region and regulate the transcription of genes such as c-myc, platelet-derived growth factor (PDGF)A and p53 (Postel and Ferrone, 1994; Postel et al., 2000; Cho et al., 2001; Raveh et al., 2001; Ma et al., 2002; Cervoni et al., 2003; Arnaud-Dabernat et al., 2004; Cervoni et al., 2006). Binding of either isoform to the PDGF-A promoter leads to suppression of PDGFA's transcription (Ma et al., 2002). One study also found that cytosolic NDPKs bind in vivo to the promoter of NDPK-A (Cervoni et al., 2006), suggesting that the transcription of these proteins is subject to feedback regulation. Some isoforms - NM23-H1, NM23H5, NM23-H7, and NM23-H8 - have 3' to 5'-exonuclease activity (Yoon et al., 2005; Kaetzel et al., 2006). For example, NDPK A is involved in granzyme A-induced cell death (Fan et al., 2003; Martinvalet et al., 2005), acting as an exonuclease to create single-strand nicks in DNA (Chowdhury et al., 2006) and leading to apoptosis.

\subsubsection{Intracellular traffic}

NDPKs have been reported to play an important role in endocytosis. The Drosophila NDPK homolog, Awd enhances dynamin-mediated endocytosis, presumably by supplying GTP for dynamin, a GTPase (Krishnan et al., 2001). In MDCK cells, NDPK

A is recruited by Arf6-GTP to areas of cell-cell contact where it promotes dynamin- 
dependent vesicle fission of clathrin-coated vesicles and downregulates Rac1 (Palacios et al., 2002). NDPK A is also essential for von Hippel-Lindau tumor suppressor proteinmediated endocytosis of the fibroblast growth factor (FGF) receptor 1 (Dammai et al., 2003; Hsu et al., 2006). In addition, NDPK B contributes to the formation of the cytosolic coat protein complex II (COPII), which coats vesicles traveling from the endoplasmic reticulum (ER) to the Golgi apparatus (Kapetanovich et al., 2005).

\subsubsection{Signal transduction}

Members of the NDPK family also participate in signal transduction (reviewed in Otero, 2000). The Otero lab found that NDPK plays a role in the regulation of the muscarinic $\mathrm{K}^{+}$channel (Xu et al., 1996). In this system, NDPK decreases the desensitization of the response of G protein-coupled inward rectifier potassium (GIRK) channel to muscarinic receptor activation (Otero et al., 1999); this function is unrelated to its kinase function. Also, NDPK B has been reported to activate the $K_{C a} 3.1$ channel (Srivastava, et al., 2006). In another example of NDPK's participation in signal transduction, both NDPK A and NDPK B have been reported to inhibit Rac1 signaling. NDPK A apparently inhibits Rac1 activation through interaction with Tiam1 (Otsuki et al., 2001; Palacios et al., 2002), while NDPK B inhibits Rac1 and Cdc42 activation (Degani et al., 2002) when it is targeted to the cell periphery by engaged $\beta_{1}$ integrins, where it complexes with the $\beta_{1}$ integrin cytoplasmic domain associated protein (ICAP) to control cell migration and spreading (Fournier et al., 2002; Fournier et al., 2003). In addition, NDPK B has also been shown to allow $\mathrm{G \alpha}_{\mathrm{q}}$-induced internalization of the thromboxane A2 receptor (TP $\beta$; Rochdi et al., 2004) by inactivating Rac1. The latter 
report potentially demonstrates an interaction between NDPK B and a GPCR signaling pathway. However, the results were obtained in cells overexpressing both the TP $\beta$ receptor and NDPK B, and may not be representative of physiological conditions. In Drosophila, Awd promotes down-regulation of surface receptors such as Pvr (plateletderived growth factor/vascular endothelial growth factor receptor); Awd acts in collaboration with dynamin, thereby regulating the chemotactic signal strength (Nallamothu et al., 2008)

NDPK A has been shown to regulate both TGF $\beta$ and p53 through its interactions with serine/threonine receptor associated protein (STRAP; Jung et al., 2007; Seong et al., 2007), while NDPK B is associated with estrogen receptor- $\beta$ (ER $\beta$ ), which may play a role in cell migration (Rayner et al., 2007; Rayner et al., 2008). Putative interactions with GTP binding protein $\beta \gamma$ subunits and GPCRs have also been described, and are reviewed in Hippe and Wieland (2006) and Wieland (2007).

\subsection{Summary}

Only a small part of the literature on NDPK/NM23 is cited here. However, it is clear that NDPKs are not just housekeeping enzymes, and have multiple roles in cells. The challenge is to determine how these proteins accomplish such a wide array of tasks. The work described here is based on the idea that a protein may have numerous roles depending on its location in the cell, and affect different steps of cellular events in these various locations. Determining the subcellular localization of cytosolic NDPKs and how it is achieved is an essential step in deciphering how they perform their diverse functions. 


\section{CHAPTER 2: Experimental Methods}

\subsection{Cell culture and transfection}

NIH-3T3 fibroblasts and Madin-Darby Canine Kidney (MDCK) cells were grown in Dulbecco's modified Eagle's medium supplemented with $10 \%$ fetal bovine serum, 2 $\mathrm{mM}$ L- glutamine, $1 \mathrm{mM}$ sodium pyruvate, $100 \mathrm{U} / 1$ penicillin and $100 \mu \mathrm{g} / \mathrm{ml}$ streptomycin at $37^{\circ} \mathrm{C}$, in a humidified $5 \% \mathrm{CO}_{2}$ atmosphere.

In studies of the microtubule-associated vesicle fraction $(\mathrm{Mt} / \mathrm{Ves})$ cells were subcultured at 50-70\% confluence; assays were performed between passages 2 and 8 . For nucleotide depletion experiments, cultures were serum-deprived for 16 hours. Cells were either left untreated in controls or exposed to treatments as indicated. Controls for deoxyglucose/azide treatment were kept in low glucose medium.

Primary cilia experiments on NIH-3T3 cells were performed in cells at passages 4-7 that were $60-80 \%$ confluent and serum-deprived for $48-72$ hours, all conditions that increase expression and length of primary cilia in this cell line (Tucker et al., 1983; Alieva et al., 1999). Xenopus A6 cells were grown at $28^{\circ} \mathrm{C}$ in $1 \% \mathrm{CO}_{2}$, in a medium composed of $40 \%$ Leibowitz L-15, 17\% F-12K Nutrient Mixture (Kaighn's modification), 33\% water, $8 \%$ fetal bovine serum, $1.6 \mathrm{mM}$ L-glutamine, $80 \mathrm{U} / \mathrm{L}$ penicillin and $80 \mu \mathrm{g} / \mathrm{ml}$ streptomycin. Unless otherwise stated, A6 cells were cultured for 7-9 days in complete medium after reaching confluence before fixation and staining.

Transfections were performed using LipofectAMINE-2000 as recommended by the manufacturer. The EGFP fusion constructs used (courtesy of Dr. Marie-Lise Lacombe, INSERM, France) were made using Clonetech Living Colors vectors, and 
consist of the vector pEGFP-N1 (control), pEGFP-N1-H1 (NDPK A) and pEGFP-N1-H2 (NDPK B), with the coding sequence for NDPKs inserted at the N-terminus of the EGFP coding sequence. The resulting protein fusions are expressed under the control of the strong CMV promoter. The EGFP variant of GFP has enhanced fluorescence compared to GFP (35-fold increase) and the fusion proteins show greater solubility when expressed in mammalian cells. Fusions to the N-terminus of EGFP retain the fluorescent properties of the native protein, allowing the localization of the fusion protein in vivo.

\subsection{Microscopy methods}

\subsubsection{Indirect immunofluorescence}

Cells were grown on No. 1 thickness acid-washed glass coverslips and were fixed for 10 minutes at room temperature in $4 \%$ paraformaldehyde $/ 0.1 \%$ glutaraldehyde, followed by 3 rinses with PBS. Free aldehyde groups were quenched with $\mathrm{NaBH}_{4}$ for 5 minutes, rinsed 3 more times with PBS, and transferred to a blocking solution composed of $0.1 \%$ saponin and $3 \%$ BSA in PBS for 1 hour. This same buffer was used to dilute primary and secondary antibodies. Coverslips were incubated overnight with primary antibodies at $4{ }^{\circ} \mathrm{C}$, washed 4 times 5 minutes with PBS and incubated for 45 minutes at room temperature with secondary antibodies. After four additional washes with PBS and 1 rinse with water, coverslips were mounted in Mowiol (Calbiochem) containing 2\% $n$ propylgallate. For NDPK detection in NIH-3T3 cells, we utilized a monoclonal antibody specific for NDPK A (NM301) from Santa Cruz Biotechnology, and a polyclonal antibody, Ab-1 (Labvision) that recognizes human NDPKs A and B (amino acids 86102). Polyclonal antibodies to frog NDPK were used to detect NDPK in A6 cells (Yi et 
al., 1995; Otero, 1997). Secondary antibodies (multiple labeling grade) used for immunofluorescence were from Jackson Immunoresearch Laboratories (West Grove, PA; Texas Red conjugates) and Molecular Probes (Eugene, OR; Alexa 488 conjugates).

Flow fixation of A6 cells, a procedure that aligns and flattens cilia, was performed essentially as described by Wheatley and Bowser (2000). Coverslips were propped at an angle, and fixation solution was repeatedly applied to the top of the coverslip to fix the primary cilia in a straightened manner, as if they had been "combed".

Following primary cilia isolation (described in 2.4.2) the fraction enriched in cilia was placed on a glass coverslip, dried with a stream of nitrogen, fixed and stained as above.

Coverslips were examined on a Nikon Diaphot microscope equipped with 40X and 100X oil immersion objectives. Digital images were obtained with a Nikon CoolPix 990 camera. Figures were assembled using Adobe Photoshop software. To ensure that the images of cilia were accurate, in particular for length measurement, 3-5 pairs of throughfocus images of acetylated $\alpha$-tubulin and NDPK staining were obtained from each field. Measurements of apparent ciliary length were performed on magnified images using Corel Draw.

For confocal microscopy, a $10 \mu \mathrm{l}$ aliquot of $\mathrm{Mt} / \mathrm{Ves}$ was spread onto polylysinecoated coverslips and allowed to adhere for 3 minutes. The specimen was fixed and processed for indirect immunofluorescence as above, using an antibody to NDPK (Ab-1; 1:50), followed by Texas Red anti-rabbit (1:100). Confocal and differential interference 
contrast (DIC) images were acquired with an Olympus FluoView 300 confocal laser scanning microscope (Olympus, Center Valley, PA).

Measurements of the diameters of NDPK-labeled vesicles were performed in images of preparations immunolabeled with Ab-1 or NM301. Histograms were constructed in Origin 7.0.

\subsubsection{Electron microscopy}

Formvar-coated carbon grids (200-mesh) were floated on drops of a primary cilium suspension for 2 minutes. The grids were stained with 2\% uranyl acetate and examined on a Phillips electron microscope at $80 \mathrm{kV}$.

\subsection{Biochemical methods}

\subsubsection{SDS-PAGE and immunoblotting}

Samples solubilized by sonication for 10 minutes in urea SDS-PAGE sample buffer (4.6\% SDS, $8 \mathrm{M}$ urea, $150 \mathrm{mM}$ Tris $\mathrm{pH} 8.0,0.1 \%$ bromophenol blue, $20 \mathrm{mM}$ DTT) were alkylated with $60 \mathrm{mM}$ iodoacetamide and resolved in standard $15 \%$ or $4-20 \%$ Tris-Glycine gels, or in NuPage gels, 10 or $12 \%$, run in MOPS/SDS buffer. The resolved proteins were then transferred to nitrocellulose membranes. Blots were rinsed in PBS, blocked in $5 \%$ milk/PBS with $0.01 \%$ thimerosal for 2 hours at room temperature or overnight at $4^{\circ} \mathrm{C}$. Primary antibodies were diluted in 5\% BSA/PBS and incubated with membranes while rocking for 2 hours at room temperature. This was followed by 4 rinses in PBS, 5 minutes each. Secondary antibodies were diluted in the same buffer and applied to blots for 1 hour at room temperature. Finally, the membranes were rinsed an additional 
4 times in PBS and developed using enhanced chemiluminescence or the Licor Odyssey infrared imaging system.

Rabbit polyclonal antibodies to NDPK $\mathrm{A}$ and $\mathrm{C}$ and the affinity-purified polyclonal antibodies to NDPK B used in immunoblotting were a generous gift of Dr. Ioan Lascu (Université de Bordeaux, France); IgGs cross-reacting with NDPK A were removed from the latter by chromatography on columns of immobilized NDPK A (Phang-Ba et al., 1998). HRP-labeled secondary antibodies for immunoblotting were from KPL (Gaithersburg, MD). Antibodies labeled with infrared dyes (IRDye $800 \circledast$ antirabbit and anti-goat, IRDye $700 \circledast$ anti-mouse) were from Rockland Immunochemicals.

Anti-Arf6, anti-Rac1 and anti-von Hippel-Lindau protein were from Santa Cruz Biotechnology, and anti-dynamin (Ab-1) from Calbiochem. Other antibodies used were: polyclonal anti-Rab4 (Santa Cruz and StressGen), monoclonals anti-LAMP-1 and antikinesin heavy chain (1D4B and SUK4, respectively), from the Developmental Studies Hybridoma Bank, Iowa, and monoclonal anti- $\alpha$-tubulin (clone DM1A) from SigmaAldrich. The monoclonal anti-dynein antibody (74.1) was a generous gift from Dr. K. Kevin Pfister, University of Virginia.

\subsubsection{NDPK enzyme assay}

NDPK activity was measured using a coupled assay as in Lascu et al. (1983). The assay uses ATP as a phosphate donor and dTDP as a phosphate acceptor as shown below.

$$
\begin{gathered}
\mathrm{ATP}+\mathrm{dTDP} \underset{\mathrm{NDPK}}{\longrightarrow} \mathrm{ADP}+\mathrm{dTTP} \\
\mathrm{ADP}+\mathrm{PEP} \underset{\mathrm{PK}}{\longrightarrow} \mathrm{ATP}+\text { pyruvate } \\
\text { pyruvate }+\mathrm{NADH} \underset{\mathrm{LDH}}{\longrightarrow} \text { lactate }+\mathrm{NAD}
\end{gathered}
$$


dTDP is used as the phosphate acceptor because it is a poor substrate for pyruvate kinase (PK) and a good substrate for NDPK, guaranteeing a low background rate. The rate of ADP formation can be determined by following the decrease in optical absorbance at $340 \mathrm{~nm}$, since NADH, but not NAD, absorbs light at that wavelength.

For the assay, a reaction mixture containing $50 \mathrm{mM}$ Tris, $\mathrm{pH} 7.5,5 \mathrm{mM} \mathrm{MgCl} 2$, $75 \mathrm{mM} \mathrm{KCl}, 1 \mathrm{mg} / \mathrm{ml} \mathrm{BSA}, 1 \mathrm{mM}$ phosphoenol pyruvate, $0.1 \mathrm{mM} \mathrm{NADH}, 1 \mathrm{mM}$ ATP, and $2 \mathrm{U} / \mathrm{ml}$ lactate dehydrogenase ( $\mathrm{LDH})$ and pyruvate kinase (PK) was placed in a cuvette. The sample was added and the basal rate that reflects the ATPase activity of the sample, as well as the background NDPK activity of the coupling enzymes, was recorded. dTDP $(0.2 \mathrm{mM})$ was then added, and the decrease in NADH concentration was measured at $340 \mathrm{~nm}$ over a period of 2 minutes. The $\Delta \mathrm{OD} / \mathrm{min}$ was calculated during the linear phase at the beginning of the reaction, and converted into units ( $\mu$ moles of substrate transformed/min) $/ \mathrm{mg}$ of protein.

\subsection{Isolation of subcellular fractions}

\subsubsection{Isolation of microtubule-associated endocytic vesicles and proteins}

Microtubule-associated endocytic vesicles (Mt/Ves) were isolated (summarized in Scheme 1) by the method of Wolkoff and colleagues (Goltz et al., 1992, Oda et al., 1995). NIH-3T3 cells were homogenized in MEPS (35 mM PIPES, pH 7.1 with $\mathrm{NaOH}, 5 \mathrm{mM} \mathrm{MgSO}_{4}, 5 \mathrm{mM}$ EGTA, $200 \mathrm{mM}$ sucrose, $1 \mathrm{mM}$ DTT; calculated free $\mathrm{Mg}^{2+}$ is $4.3 \mathrm{mM}$ ) containing protease inhibitors ( $2 \mathrm{mM}$ PMSF, $1 \mathrm{mM}$ benzamidine, $2 \mu \mathrm{g} / \mathrm{ml}$ leupeptin). A post-nuclear supernatant (S1) was centrifuged at 40,000 $\mathrm{xg}$ for 20 minutes. 
The supernatant (S2; small vesicles and cytosol) was incubated at $37^{\circ} \mathrm{C}$ for 30 minutes with $20 \mu \mathrm{M}$ taxol (and nucleotides when indicated) to polymerize tubulin and pelleted at $16,000 \mathrm{xg}$ for 30 minutes at $4{ }^{\circ} \mathrm{C}$. The pellets were brought to the original volume with MEPS, $20 \mu \mathrm{M}$ taxol was added and the samples were centrifuged at 16,000 $\mathrm{xg}$. After a second wash, the pellets were solubilized in SDS-PAGE sample buffer and analyzed by SDS-PAGE and immunoblotting. In some instances the pellets were incubated with nucleotides after two washes, and then processed for analysis. Samples were immunoblotted in parallel with antibodies to $\alpha$-tubulin (and Rab4 or LAMP1, when appropriate) to ensure treatments did not affect tubulin polymerization or co-pelleting of membrane vesicles. Results shown are representative of 3-10 separate experiments.

Cytosol from NIH-3T3 cells was prepared by centrifugation of the S2 supernatant in a TLA 100.3 rotor (Beckman Coulter) at $338,000 \mathrm{xg}$ for 1 hour at $4{ }^{\circ} \mathrm{C}$.

To determine whether NDPK is initially associated with vesicles or soluble tubulin, the supernatant of the $40,000 \mathrm{xg}$ step (S2) was centrifuged at $230,000 \mathrm{xg}$ for 1 hour in a TLA 100.3 rotor to sediment membrane vesicles. The supernatant was incubated with taxol as described above to polymerize soluble tubulin, and centrifuged at $16,000 \mathrm{xg}$ (Scheme 2). Both pellets were washed twice with MEPS and analyzed by immunoblotting.

\subsubsection{Isolation of primary cilia}

Primary cilia were isolated from A6 cells grown in $150 \mathrm{~mm}$ dishes. Cells were rinsed gently with PBS, layered with $10 \mathrm{ml}$ PBS and the plates were placed in a rotary shaker at $360 \mathrm{rpm}$ for 4 minutes at room temperature. To monitor deciliation as well as 

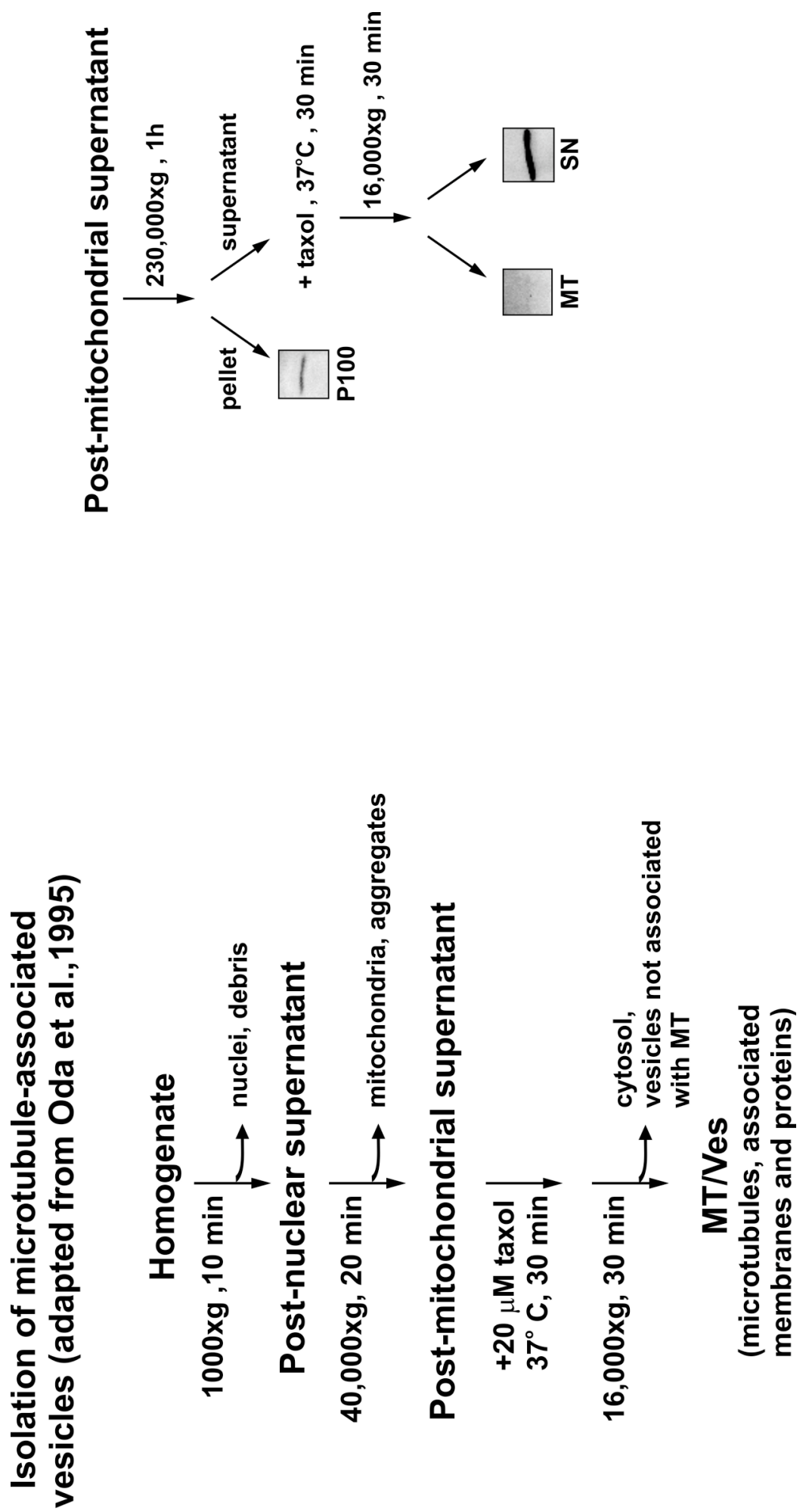
cell integrity, cells grown on coverslips were shaken under the same conditions, fixed and stained. The supernatants were removed and centrifuged at $1000 \mathrm{xg}$ for 10 minutes to remove cells and debris. The cilia were collected by centrifugation at $40,000 \mathrm{xg}$ for 30 minutes. Pellets were suspended in $20 \mathrm{mM}$ Tris- $\mathrm{HCl}, \mathrm{pH} 8,50 \mathrm{mM} \mathrm{KCl}, 4 \mathrm{mM} \mathrm{MgSO}_{4}, 1$ mM DTT, 0.5 mM EDTA (resuspension buffer, RB; Hastie et al., 1986) and analyzed by immunoblotting. Six to $20 \mu \mathrm{g}$ of isolated primary cilia were obtained using four $150 \mathrm{~mm}$ dishes.

To localize NDPK within primary cilia we developed a method based on isolation procedures for components of intraflagellar transport (IFT) processes (Cole et al., 1998). To disrupt the outer membrane, pellets obtained at 40,000 $\mathrm{xg}$ were suspended in $0.5-1 \mathrm{ml}$ of $\mathrm{RB}$ buffer, frozen in liquid nitrogen, thawed and centrifuged at $16,000 \mathrm{xg}$ for 30 minutes. The resulting supernatant is the matrix, including soluble proteins. To remove proteins bound to the preparation in a nucleotide-sensitive manner, the pellet, which now contained axonemal and membrane proteins, was extracted with $10 \mathrm{mM}$ ATP in RB for 10 minutes on ice, and centrifuged as above. Membrane proteins in the ATP-stripped pellet were then solubilized with $0.5 \% \mathrm{w} / \mathrm{v}$ Triton $\mathrm{X}-100$ in RB on ice for 10 minutes. The final pellet contained the detergent-insoluble axonemal fraction.

For electrophoresis, TCA-precipitated supernatants and pellets were solubilized as described above and resolved in $15 \%$ or $4-20 \%$ minigels. Proteins were transferred to nitrocellulose and immunoblotted. NDPK was detected with antibodies to frog NDPK. Acetylated $\alpha$-tubulin was detected with a monoclonal antibody (clone 6-11B-1) from Zymed. Individual band density was quantified using Maxim DL 3.0 (Diffraction 
Limited). Protein concentration was determined by the BCA assay (Pierce). Enzyme activity was determined in triplicate by the coupled assay as described above (Section 2.3.2).

\subsection{Protein purification}

\subsubsection{Purification of NDPK from human erythrocytes}

Soluble NDPK was purified from human erythrocytes using a modification of an earlier protocol (Yi et al., 1995). Cells were lysed in buffer A (25 mM MOPS, pH 7.5, 1 $\mathrm{mM}$ EDTA, $1 \mathrm{mM}$ PMSF) on ice, and centrifuged at 17,600 $\mathrm{xg}$ for 1 hour. The supernatant was removed and precipitated with $45 \%$ ammonium sulfate. The suspension was centrifuged for 10 minutes at $10,000 \mathrm{xg}$; the supernatant was decanted, and centrifuged again for 10 minutes at $10,000 \mathrm{xg}$. The supernatant was brought to $85 \%$ ammonium sulfate and centrifuged for 30 minutes at $10,000 \mathrm{xg}$. The pellet was suspended in buffer A and dialyzed to the same buffer for 48 hours and concentrated on an Amicon XM50 membrane. The sample was centrifuged for 15 minutes at 48,000 xg, and applied to a Reactive Yellow 3 column $(2.5 \mathrm{~cm}$ diameter x $17.5 \mathrm{~cm}$ height). The column was washed with buffer $\mathrm{B}$ (buffer A with $0.15 \mathrm{M} \mathrm{NaCl}$ ) until the $\mathrm{A}_{280}$ returned to the baseline value. NDPK was eluted with $0.5 \mathrm{mM}$ ATP in buffer B; the elution of protein was followed at $290 \mathrm{~nm}$ to minimize interference by ATP (Fig. 2a). Fractions were assayed for NDPK activity, pooled and concentrated by ultrafiltration in a stirred cell (Amicon) fitted with a YM30 membrane. The purified enzyme was stored at $-20{ }^{\circ} \mathrm{C}$ in $\mathrm{PBS} / 50 \%$ glycerol. Analysis by SDS-PAGE (Fig. 2b) indicated that the preparation 
$\boldsymbol{m}$
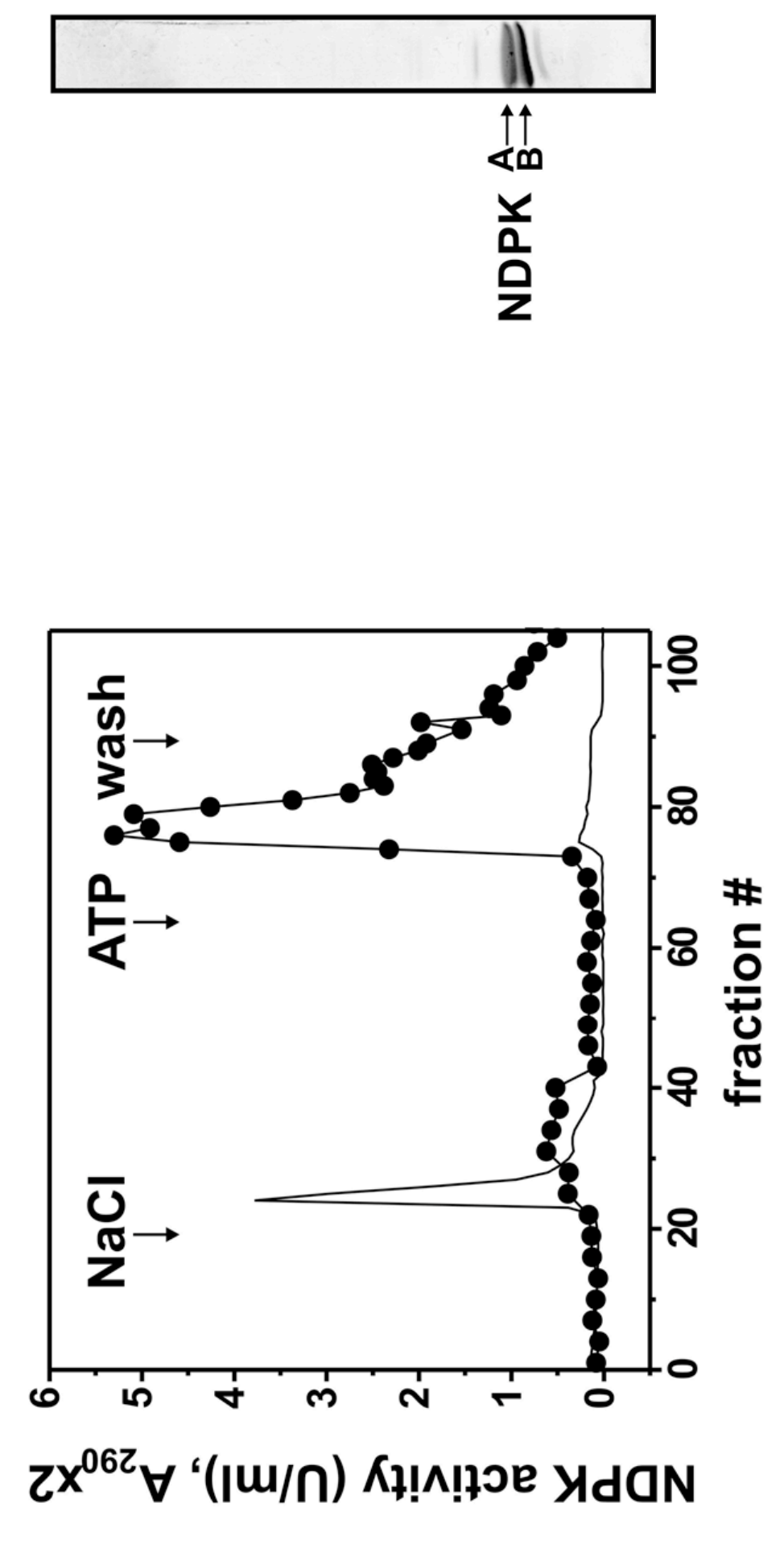

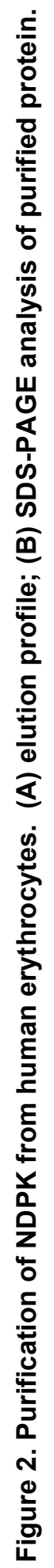


was $88 \%$ pure, and composed of two polypeptides, NDPK A (20.5 kDa) and B (19 kDa). The specific activity was $1,200 \mathrm{U} / \mathrm{mg}$ when tested by the coupled assay.

\subsubsection{Bacterial expression and purification of human NDPK A}

Wild-type NDPK A was expressed in E. coli BL21 (DE3) from a pET 21b vector generously provided by Dr. Marie-Lise Lacombe (INSERM U 402, Paris, France). Selection was made in LB medium with $0.1 \mathrm{mg} / \mathrm{ml}$ ampicillin. Cultures derived from a single colony were grown at $37{ }^{\circ} \mathrm{C}$ in $\mathrm{LB}$ medium with $0.2 \mathrm{mg} / \mathrm{ml}$ ampicillin to an $\mathrm{OD}_{550}$ of 0.6 , when protein expression was induced with $0.4 \mathrm{mM}$ isopropyl-beta-Dthiogalactopyranoside (IPTG). After 3.5 hours the bacteria were harvested and pelleted at $5,000 \mathrm{xg}$ for 10 minutes at $4{ }^{\circ} \mathrm{C}$. The pellets were resuspended in buffer A $(50 \mathrm{mM}$ Trisacetate, $\mathrm{pH}$ 8.0, 1 mM EDTA, $1 \mathrm{mM}$ DTT) and frozen in liquid nitrogen.

For purification of expressed protein (modified from Lascu et al., 1997), one bacterial pellet $(1 \mathrm{~g})$ was thawed in $20 \mathrm{ml}$ of B-Per (Pierce) containing $1 \mu \mathrm{g} / \mathrm{ml}$ each of aprotinin and leupeptin, $2 \mu \mathrm{g} / \mathrm{ml}$ of pepstatin, $1 \mathrm{mM}$ benzamidine and $1.25 \mathrm{mM}$ PMSF, and stirred for 10 minutes at room temperature. The mixture was centrifuged for 15 minutes at 17,600 $\mathrm{xg}$. The clear supernatant was diluted 1:1 with buffer A and applied at $2 \mathrm{ml} / \mathrm{min}$ to $2 \times 5 \mathrm{ml} \mathrm{Q}$ Econopak cartridges in tandem (Bio Rad Laboratories). The column was washed with 12 volumes of buffer A (fraction Q1) and eluted with a gradient

of 0 to $0.5 \mathrm{M} \mathrm{NaCl}$ in buffer A (fraction Q2). The fractions were monitored by $\mathrm{A}_{280}$ and NDPK activity. Fractions with high specific activity were pooled and applied to $2 \times 5 \mathrm{ml}$ Econopak Blue cartridges $(10 \mathrm{ml})$. The column was washed with 10 volumes of buffer B (buffer A with $0.15 \mathrm{M} \mathrm{NaCl}$ ) and eluted successively with 2 volumes each of buffer B 
containing $1 \mathrm{mM}$ ATP, buffer A with $0.5 \mathrm{M} \mathrm{NaCl}$, and buffer A with $2 \mathrm{M} \mathrm{NaCl}$. Elution of protein was followed at $290 \mathrm{~nm}$. Fractions with high specific NDPK activity were pooled, concentrated and desalted in a Centricon 30 (Amicon), dialyzed overnight to PBS, and frozen in aliquots in liquid nitrogen. The purified protein was essentially pure, as judged by SDS-PAGE (Fig. 3) and had a specific activity of $1600 \mathrm{U} / \mathrm{mg}$. Note that after elution with ATP, NDPK A runs as a doublet. This behavior arises because the phosphorylated form migrates slower than the free enzyme.

\subsection{Size exclusion chromatography}

Mt/Ves pellets were isolated and incubated with or without $1 \mathrm{mM} \mathrm{GTP.} \mathrm{After}$ centrifugation at $16,000 \mathrm{xg}$ the supernatant was removed and applied to a Superose 12 HR 10/30 (Amersham Biosciences) column at a flow rate of $0.4 \mathrm{ml} / \mathrm{min}$ and fractions of $1.0 \mathrm{ml}$ were collected. The protein elution profile was monitored at $280 \mathrm{~nm}$, and fractions were assayed for NDPK activity. The samples were precipitated with trichloroacetic acid, dissolved in sample buffer, and analyzed by SDS-PAGE followed by silver staining and/or immunoblotting. The column was calibrated with standards (IgG, BSA, $\beta$ lactoglobulin, cytochrome $\mathrm{C}$ and vitamin B12) according to the manufacturer's instructions. Similar results were obtained with a calibrated Sepharose 6 HR 10/30 column, which has a higher exclusion limit.

\section{$2.7\left[\alpha-{ }^{32} P\right] G T P$ overlay assay}

Samples were resolved by SDS-PAGE and transferred to nitrocellulose. The membrane was rinsed and pre-incubated for 1 hour in $50 \mathrm{mM}$ Tris, $\mathrm{pH}$ 7.4, 0.3\% Tween20, $5 \mathrm{mM} \mathrm{MgCl} 2$, and $100 \mathrm{mM}$ ATP. $\left[\alpha_{-}{ }^{32} \mathrm{P}\right] \mathrm{GTP}$ was added to a final concentration of 


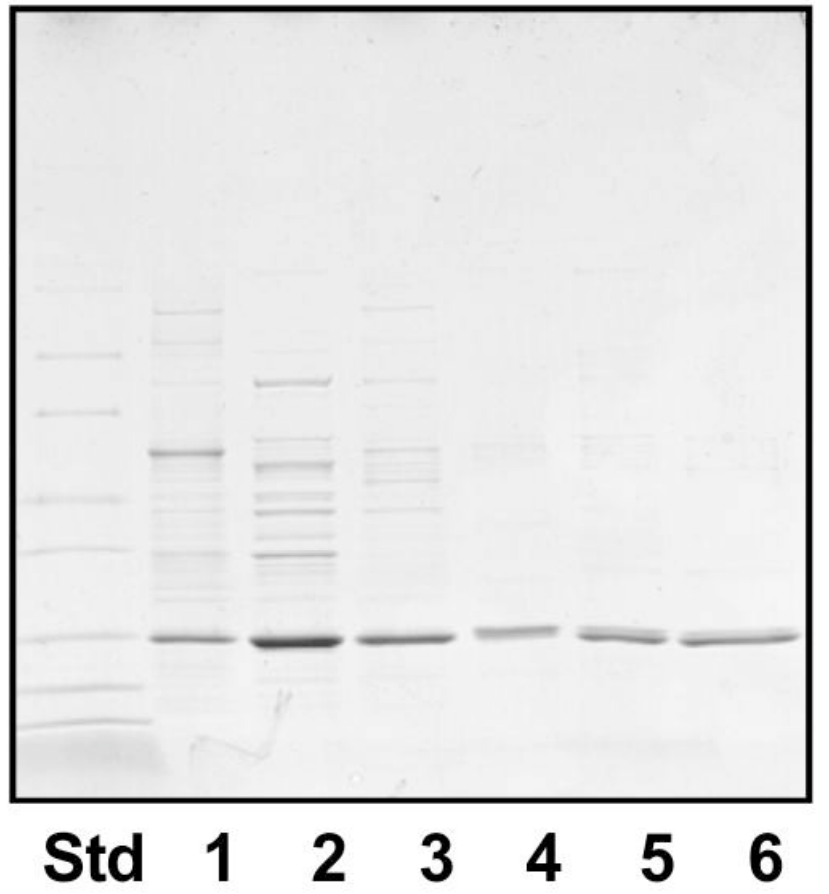

Figure 3. Purification of human NDPK A. Lane 1 - Extract (sample applied to Q); 2 - Q1 (wash); 3 - Q2 (applied to Blue); 4 - Blue 1 (ATP); 5 - Blue 2 (0.5 M NaCl); 6 - Blue 3 (2M NaCl) 
$1 \mu \mathrm{Ci} / \mathrm{ml}$, and the blot was incubated for 1 hour at room temperature, then rinsed 4 times for 10 minutes each in pre-incubation buffer and dried. Labeled proteins were detected with X-ray film exposed to blots at $-80{ }^{\circ} \mathrm{C}$ for $1-3$ days.

\subsection{Protein-lipid overlay assay.}

Nitrocellulose membranes spotted with phospholipids (PIP strips ${ }^{\mathrm{TM}}$, Echelon Biosciences) were blocked for 1 hour at $25^{\circ} \mathrm{C}$ or overnight at $4{ }^{\circ} \mathrm{C}$ with $3 \%$ bovine serum albumin (BSA) in Tris-buffered saline with $0.1 \%$ Tween-20 (TBST). The blots were then overlaid with either cytosol from NIH-3T3 cells or $3.75 \mu \mathrm{g} / \mathrm{ml}$ purified NDPK A, with or without GTP, for 1 hour at room temperature. The membranes were washed 6 times for 5 minutes with TBST, and then incubated with primary antibody against NDPK for 1 hour. After 4 washes, the blot was incubated with secondary antibody for 1 hour, washed 4 more times, and anti-NDPK antibodies were detected using enhanced chemiluminescence.

\subsection{Liposome binding assays}

The lipid combinations used are listed in Table 1. Phospholipids [dioleolylphosphatidylcholine (DOPC), dioleoylphosphatidylethanolamine (DOPE), dioleoylphosphatidylserine (DOPS), 1,2-dioleoyl-sn-glycero-3-phosphate (DOPA), 1,2dioleoylphosphatidylinositol (DOPI) and carboxyfluorescein-labeled DOPE] were from Avanti Polar Lipids. Cholesterol and sphingomyelin were from Sigma Aldrich.

Small unilamellar vesicles were prepared by mixing the appropriate combination of lipid stock solutions in chloroform in a glass tube. The solvent was evaporated under a stream of nitrogen and the samples were further dried in a SpeedVac for 1 hour. Lipids 


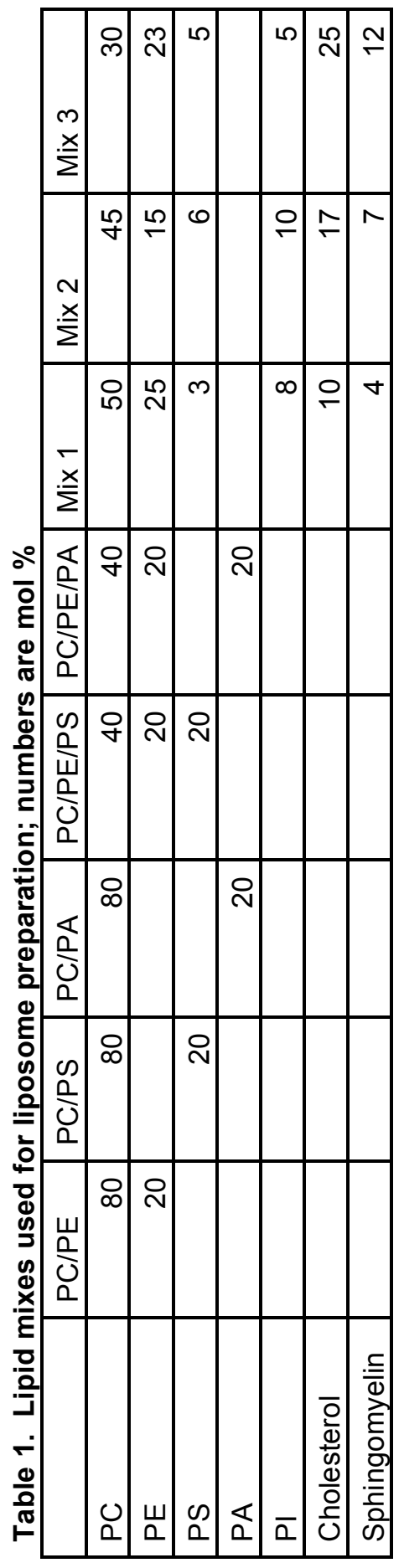


$(1.8 \mu \mathrm{mol})$ were resuspended in a vortex mixer with $90 \mu \mathrm{l}$ of MEPS buffer with $400 \mathrm{mM}$ sucrose, incubated for 1 hour at room temperature, and sonicated on ice in a bath sonicator (Model G112SPIT, Laboratory Supply Company, Hicksville, NY) for 8-12 minutes. Prior to experiments, cytosol or purified proteins were diluted in MEPS with $100 \mathrm{mM}$ sucrose and centrifuged in a Beckman Airfuge at 167,000 xg for 10 minutes to remove aggregates. Liposome suspensions were mixed with cytosol or purified NDPK, incubated at $30{ }^{\circ} \mathrm{C}$ for 30 minutes and pelleted by centrifugation at $200,000 \mathrm{xg}$ for 30 minutes at $25{ }^{\circ} \mathrm{C}$ in a TLA 120.1 rotor (Beckman Coulter). The supernatant was removed, the pellets were resuspended in MEPS buffer with or without $1 \mathrm{mM} \mathrm{GTP}$, and the samples were incubated for 30 minutes at $30{ }^{\circ} \mathrm{C}$ and pelleted as above. The supernatants and pellets were resolved by SDS-PAGE and immunoblotted. Lipid recovery was uniform, as established using carboxyfluorescein-labeled phosphatidylethanolamine (PE) $(0.2 \mathrm{~mol} \%)$ as a marker. 


\section{CHAPTER 3: NDPK Localizes to Vesicles Associated with Microtubules}

\subsection{Abstract}

Cytoplasmic NDPK can be separated into two populations according to subcellular localization and response to extracellular stimuli. Specifically, within minutes of stimulation of resting NIH-3T3 fibroblasts with serum, growth factors or bombesin, a portion of NDPK becomes associated with membrane ruffles and lamellipodia. Transfection of cells with activated Rac1 mimics, whereas expression of dominant negative Rac1 inhibits, the effects of extracellular stimulation on the translocation of NDPK to the cell cortex.

Another pool of NDPK accumulates independently of stimulation around intracellular vesicles. This vesicle-associated pool is not affected by extracellular stimulation or by expression of Rac1 mutants, but depends on microtubule integrity and is disrupted by nocodazole. In cell-free assays NDPK is present in membranes associated with taxol-stabilized microtubules (Mt/Ves) isolated from NIH-3T3 cells. Differential interference contrast (DIC) and confocal immunofluorescence imaging of Mt/Ves show that NDPK is associated with most of the membrane vesicles bound to microtubules. NDPK dissociates from this fraction upon incubation with $1 \mathrm{mM} \mathrm{GTP}$ or ATP as well as nucleotide analogs. NDPK is the main protein released by nucleotides from Mt/Ves and is eluted in its enzymatically active, hexameric form.

At low concentrations $(1 \mu \mathrm{M})$, both ATP and GTP have a substantial stimulatory effect on NDPK binding to Mt/Ves, while ADP inhibits binding at all concentrations. This suggests that the association of NDPK with Mt/Ves is preceded by phosphate 
transfer from NTPs to the active site histidine to generate the phosphoenzyme intermediate. Indeed, there is virtually no NDPK associated with Mt/Ves fractions isolated from cells depleted of cellular NTPs by pre-treatment with 2-deoxyglucose and azide.

The fact that NDPK is present in most of the Mt/Ves indicates that NDPK interacts with a component shared by the numerous types of vesicles and organelles transported throughout the cell via microtubules, namely phospholipids. This hypothesis was confirmed by the finding that cytosolic NDPK from NIH-3T3 cells binds to individual immobilized phospholipids, as well as to liposomes of defined phospholipid composition, and this interaction is sensitive to nucleotides. NDPK purified from human erythrocytes (eNDPK), as well as recombinant human NDPK A (rNDPK), behave similarly to the cytosolic enzyme, demonstrating that no additional factors are required for binding of NDPKs to phospholipids. We conclude that soluble NDPKs can associate with biological membranes through direct binding to membrane lipids, in a dynamic process controlled by the $[\mathrm{NTP}] /[\mathrm{NDP}]$ ratio in cells. This result gives new insight on the mechanisms by which NDPK localizes to cellular organelles.

\subsection{Introduction}

NDPK A and NDPK B have all the characteristics of cytosolic proteins, with no exposed hydrophobic segments or membrane-binding motifs. Nevertheless, in many instances these proteins associate with membranes of a wide variety of intracellular compartments. Thus, cytosolic NDPKs are found at the plasma membrane where they are involved in the function of ion channels (Srivastava et al., 2006), receptor activation and 
desensitization by agonists ( $\mathrm{Xu}$ et al., 1996; Otero et al., 1999; Rochdi et al., 2004), activation of phagocyte NADPH oxidase (Mizrahi et al., 2005), dynamin-mediated endocytosis (Krishnan et al., 2001; Palacios et al., 2002; Hsu et al., 2006) and integrinmediated adhesion (Fournier et al., 2002). Soluble NDPKs also localize to endosomes (Hsu et al., 2006), membranes from the endoplasmic reticulum (Barraud et al., 2002; Kapetanovitch et al., 2005), the Golgi and vesicles budding from the trans-Golgi network (Barraud et al., 2002), as well as phagosomes (Garin et al., 2001). In some cases, the recruitment of cytosolic NDPKs to membranes was ascribed to interactions with peripheral or integral membrane proteins such as the $G$ protein transducin (Orlov and Kimura, 1998), integrin cytoplasmic domain associated protein 1- $\alpha$ (ICAP-1 $\alpha$; Fournier et al., 2002) and the potassium channel $\mathrm{K}_{\mathrm{Ca}} 3.1$ (Srivastava et al., 2006). Overexpression of cytosolic NDPKs revealed novel associations with the GTP-bound form of ARF6 (Palacios et al., 2002), the thromboxane A2 $\beta$ receptor (Rochdi et al., 2004) and vonHippel Lindau protein (Hsu et al., 2006), and these binding partners were proposed to anchor NDPK to membranes. However, in many instances the mechanism underlying the association of NDPKs with membranes remains unclear (e.g., Krishnan et al., 2001; Gallagher et al., 2003; Mizrahi et al., 2005).

A recurring feature of the processes connected to membrane-bound cytosolic NDPKs over the years is the involvement of a GTP-binding protein such as Rac1 or dynamin. These observations led to the proposal that NDPKs bind to membranes in order to rephosphorylate the GDP produced by GTPases, regenerating the GTP needed for their function (Orlov and Kimura, 1998; Krishnan et al.; 2001, Palacios et al.; 2002, Gallagher 
et al., 2003). Complex mechanisms involving direct phosphorylation of the GDP bound to GTP binding proteins by NM23/NDPK or GTP channeling between NM23/NDPK and GTP binding proteins were invoked to account for the involvement of NDPK in G protein function, but these theories are not backed up by structural or kinetic data (reviewed in Otero, 2000).

Our present results indicate that NDPK may play a role in localized regeneration of nucleotides such as CTP and GTP, mainly because the levels of nucleotides like ATP, GTP and ADP have a profound effect on the reversible interaction of soluble NDPKs with membranes. However, it appears that the nucleotide sensitivity of this process is not linked directly to the function of GTPases or enzymes involved in lipid biosynthesis; rather, it is the result of the intrinsic catalytic properties of NDPK. More importantly, we demonstrate for the first time that purified NDPK can bind directly to chemically defined phospholipid bilayers in a nucleotide-sensitive manner. Thus, cytosolic NDPKs have the ability to associate with membranes independently of other proteins. Hence, our observations reveal a novel mechanism for the reversible targeting of soluble NDPKs to intracellular membranes.

\subsection{Results}

\subsubsection{NDPK is located in microtubule-associated vesicles and this interaction requires an intact microtubule network}

In quiescent, serum-starved NIH-3T3 fibroblasts, staining of NDPK with an antibody that recognizes both NDPKs A and B (Ab-1) reveals a cytosolic distribution pattern, more intense in the thicker central area (Fig. 4, upper left panel). In many cells, 
- serum

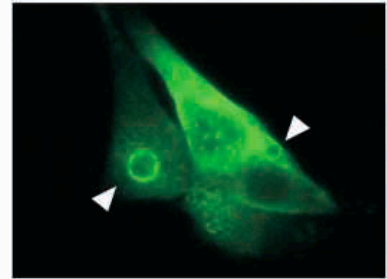

NDPK
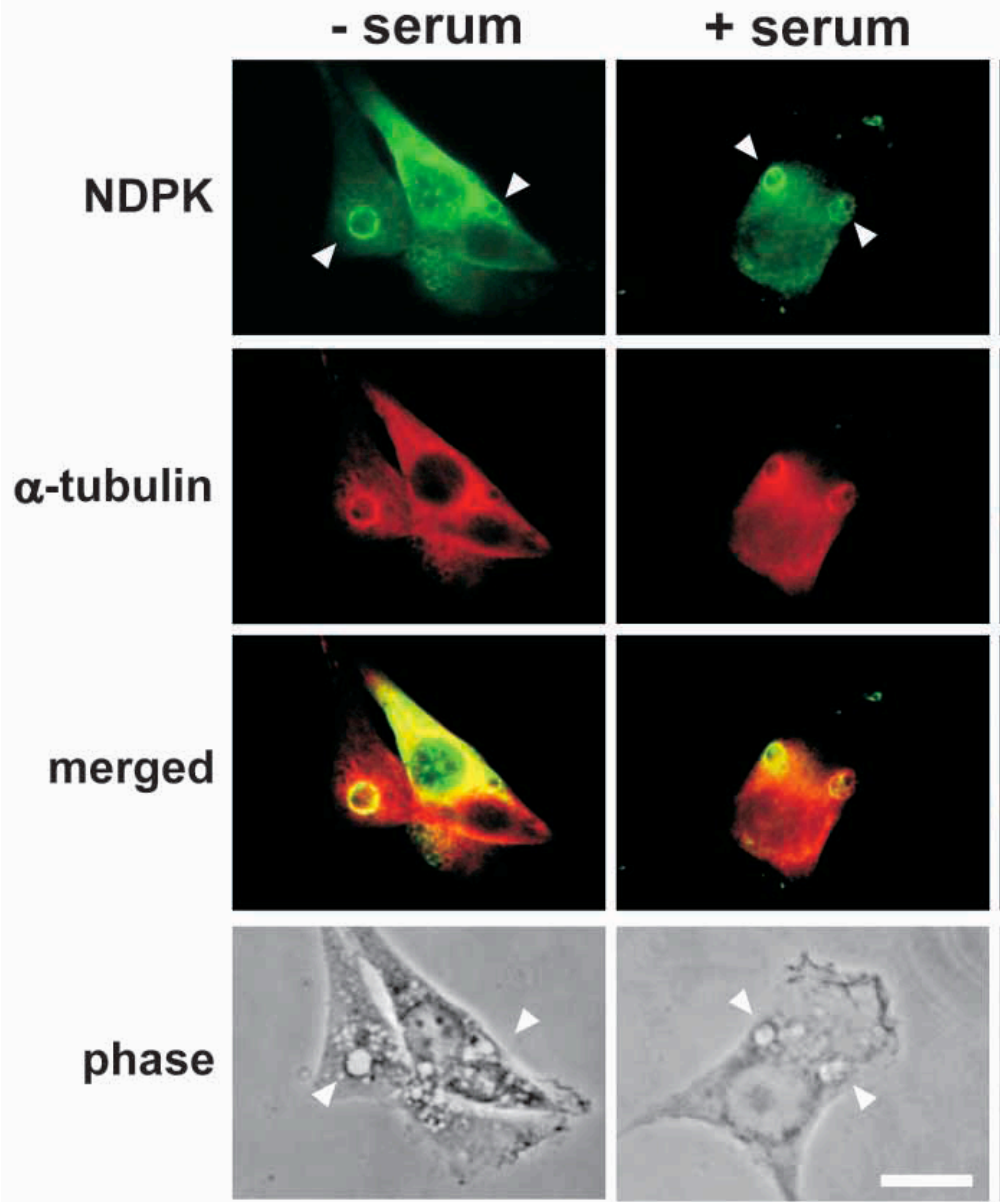

nocodazole
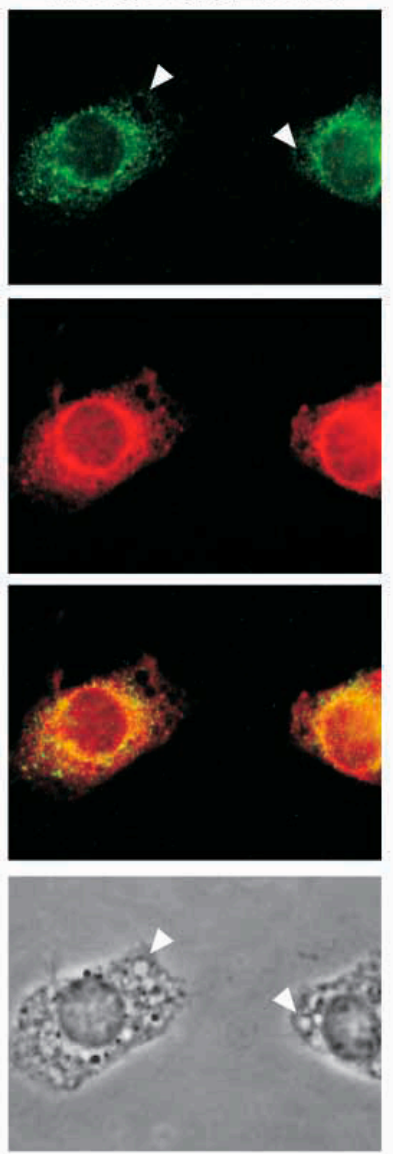

Figure 4. Localization of NDPK to microtubular-associated vesicles. Cells were fixed and double stained with antibodies to NDP kinase (Ab-1) and a-tubulin after being serum starved for 18 hours (-serum), kept in complete medium (+serum) or treated with $33 \mu \mathrm{M}$ nocodazole for 1 hour (nocodazole). Arrowheads show vesicular structures visible in phase contrast, which are labeled by antibodies to $\alpha$-tubulin and NDP kinase in serum-starved and serum-treated cells (left and middle, respectively). Labeling of vesicles is lost in cells treated with nocodazole (right). Scale bar, $20 \mu \mathrm{m}$ 
NDPK forms ring-like structures, and phase-contrast microscopy (Fig. 4, lower left panel) shows that most of these rings correspond to phase-bright vesicles of various sizes that are scattered throughout the cytoplasm, particularly around the nucleus. NDPK is associated with the outer rim of these structures, and is most conspicuous around large perinuclear vesicles (Fig. 4).

To define more clearly the mechanism by which NDPK assembles around vesicles, we first characterized their morphology and protein composition. Figure 5 shows the size distribution of NDPK-labeled vesicles, which covers a range of 0.7-6.9 $\mu \mathrm{m}$ with a median diameter of $2.3 \mu \mathrm{m} \quad(n=209)$. Given that NDPK co-localizes partially with the microtubular network in epithelial cells (Pinon et al., 1999) and the centrosome of C6 glioma cells (Roymans et al., 2000), we investigated the relationship between microtubules and the vesicles coated with NDPK by double labeling with antibodies to $\alpha-$ tubulin and NDPK (Fig. 4, left column). The signals for the two proteins overlap distinctly around the vesicles labeled by NDPK, indicating that the vesicles interact simultaneously with microtubules and NDPK. The co-localization of tubulin and NDPK at the periphery of vesicles is not a result of serum deprivation because it is also observed in cells cultured in complete medium (Fig. 4, middle column). Depolymerization of microtubules with nocodazole eliminates association of tubulin and NDPK with large intracellular vesicles (Fig. 4, right column). Thus, clustering of NDPK at the periphery of vesicles depends on an intact microtubular network.

\subsubsection{NDPK binds to membranes associated with microtubules}


Our observations are suggestive of an interaction between NDPK and microtubule-bound vesicles. To determine whether the overlap in staining reflects a physical association between NDPK and microtubule-associated vesicles, we took advantage of a cell-free assay that reproduces the in vivo association between vesicles and microtubules (Goltz et al., 1992; Oda et al., 1995), thus putting the morphological results to a biochemical test (Scheme 1). A fraction containing light membranes and cytosol was obtained from NIH-3T3 cells. Endogenous tubulin was polymerized with taxol, and pelleted at $g$ forces sufficient to sediment microtubules and associated structures, but not isolated membranes. The pellet (Mt/Ves) was washed extensively and subjected to immunoblot analysis. The Mt/Ves fraction contains NDPK, tubulin, Rab4 and LAMP-1 (Fig. 6), indicating that both early and late endosomal membranes associate with microtubules under our conditions. The microtubular motors kinesin and dynein are also present in Mt/Ves (Fig. 6), indicating that these are indeed cargo vesicles. To determine whether the NDPK pool bound to the Mt/Ves pellet was associated with microtubules or membranes, the postnuclear supernatant was centrifuged at 230,000 $\mathrm{xg}$ to sediment membranes. The supernatant (cytosol) was removed, incubated with taxol to polymerize soluble tubulin and centrifuged at $16,000 \mathrm{xg}$. When both pellets were washed and examined for their NDPK content by immunoblotting, we found that NDPK is present in the membrane pellet but not in the microtubule pellet (Scheme 2). Thus, the NDPK in the Mt/Ves pellet derives from membranes, not the tubulin component, as expected from earlier research showing that although NDPK co-immunoprecipitates from cell lysates with tubulin (Lombardi et al., 1995; Roymans et al., 2001) and co-localizes 


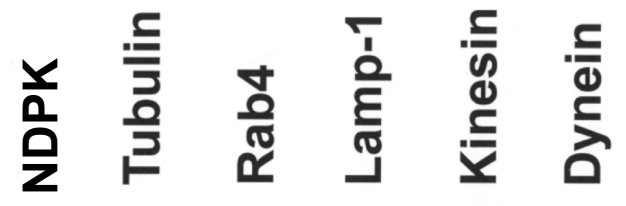

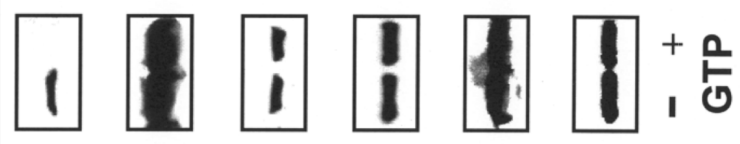

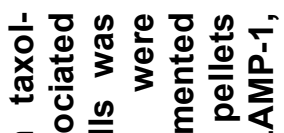
ह 융

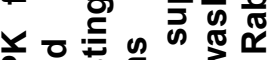

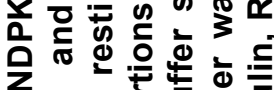

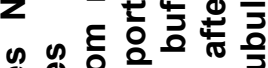

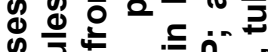

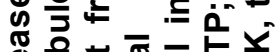
늘 苍 万人

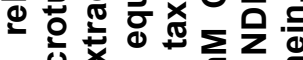
呩完

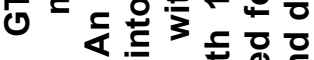

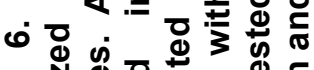

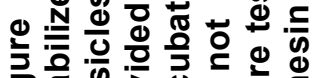

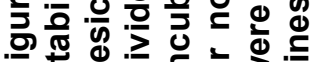
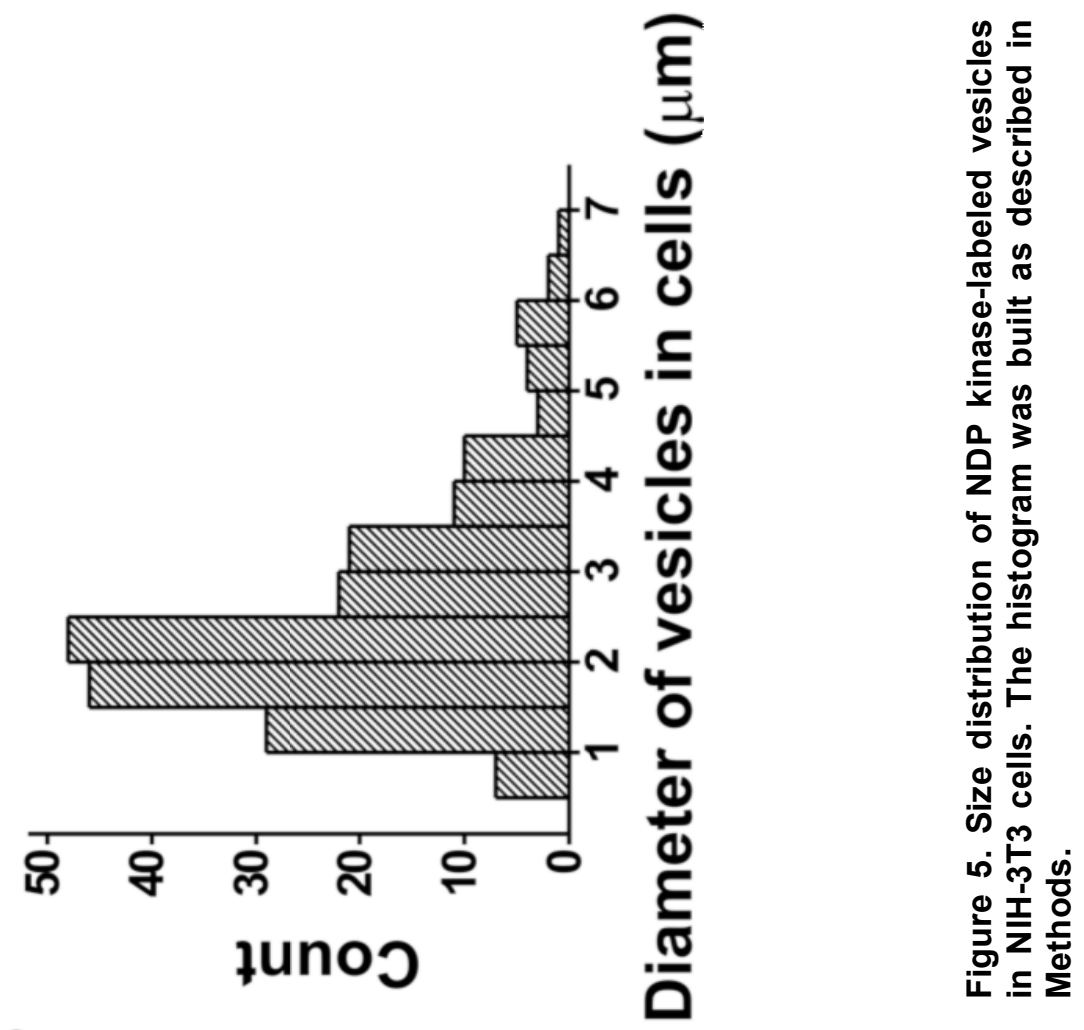
partially with microtubules in intact cells (Pinon et al., 1999), purified NDPK does not bind directly to microtubules (Melki et al., 1992; Otero, unpublished observations). Also, these results indicate that the association of NDPK with the Mt/Ves fraction is specific and not due to trapping of this abundant cytosolic protein in the microtubule pellet.

\subsubsection{NDPK localizes to the majority of vesicular structures associated with microtubules}

Analysis of the taxol-stabilized Mt/Ves fraction by confocal immunofluorescence and DIC confirms this idea. Mt/Ves pellets isolated from NIH-3T3 cells were placed on polylysine-coated coverslips, and processed for indirect immunofluorescence using an antibody to NDPK. As shown in Figure 7, NDPK localizes to numerous disk-like structures, most of them organized in linear arrays along the microtubules clearly visible in the corresponding DIC image. The heterogeneous size of the vesicles along with their irregular distribution along the microtubules is typical of similar fractions obtained from other tissue/cell types (Schroer et al. 1988; Fullerton et al., 1998; Goltz et al., 1992; Soroka et al., 1999). Comparison of histograms of the size distributions of vesicles seen in cells (Fig. 5) and in the Mt/Ves fraction (Fig. 8) shows that the two populations consist of vesicles of different median diameters, 2.3 and $0.5 \mu \mathrm{m}$, respectively. This indicates that the pool of vesicles we isolate in Mt/Ves is distinct from the population we first observed in cells using indirect immunofluorescence (Fig. 4). While the identity of the latter structures is unknown at present, the size (Fig. 8) and protein composition (Fig. 6) of the vesicles in the Mt/Ves fraction indicates that they comprise early endosomes (containing Rab4) as well as late endosomes and lysosomes (containing LAMP1), which reportedly have diameters of 0.25-0.8 $\mu \mathrm{m}$ (Novikoff et al., 1961; Roberts et al., 2000; 

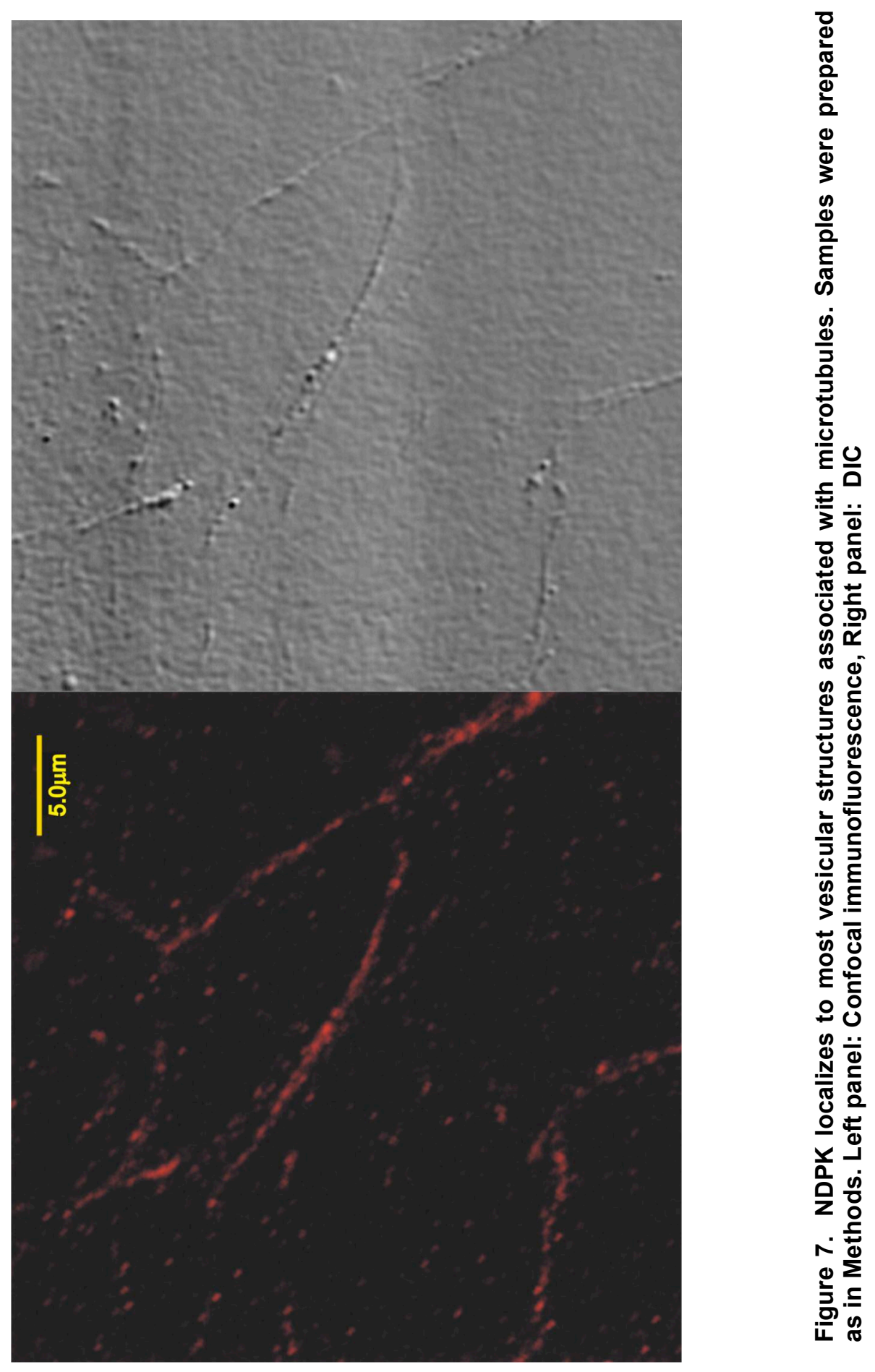
Ganley et al., 2004; Antignani and Youle, 2008). Note that smaller structures, such as clathrin coated vesicles, or those of the secretory pathway (diameters of 0.05-0.1 $\mu \mathrm{m}$; Pfeffer and Kelly, 1981; Sato and Nakano, 2007) would fall below our detection limit, which is dictated by image size and resolution. Nevertheless, our basic finding using these two approaches is the same, namely that NDPK is present at the surface of microtubule-associated membranous structures.

\subsubsection{Nucleotides release NDPK from microtubule-bound vesicles}

Formation of taxol-stabilized microtubules is usually performed in the presence of GTP, even though (at appropriate concentrations) taxol alone is sufficient to induce complete polymerization (Oda et al., 1995). Indeed, inclusion of $1 \mathrm{mM} \mathrm{GTP}$ in the incubation with taxol does not affect the amount of tubulin in the Mt/Ves pellet; neither does it affect the association of early and late endosomal membranes with the microtubule pellet, as indicated by the unchanged levels of Rab4 and LAMP-1 (Fig. 6). By contrast, GTP induces a striking release of NDPK from the pellet; ATP also reduces the association of NDPK with Mt/Ves, but is consistently $(n=6)$ slightly less effective than GTP (Fig. 9). Thus, NDPK is released from Mt/Ves through a nucleotide-sensitive site that shows some specificity for the base. The nucleotide-induced release of NDPK from the Mt/Ves fraction qualitatively resembles the behavior of the molecular motors dynein and kinesin, whose binding to microtubules and associated endosomal vesicles is also disrupted by nucleotides (Oda et al., 1995). However, we find that the amounts of dynein and kinesin associated with the Mt/Ves fraction are not altered by $1 \mathrm{mM}$ GTP (Fig. 6) or ATP (not shown). Presumably the interaction of NDPK with Mt/Ves is more 


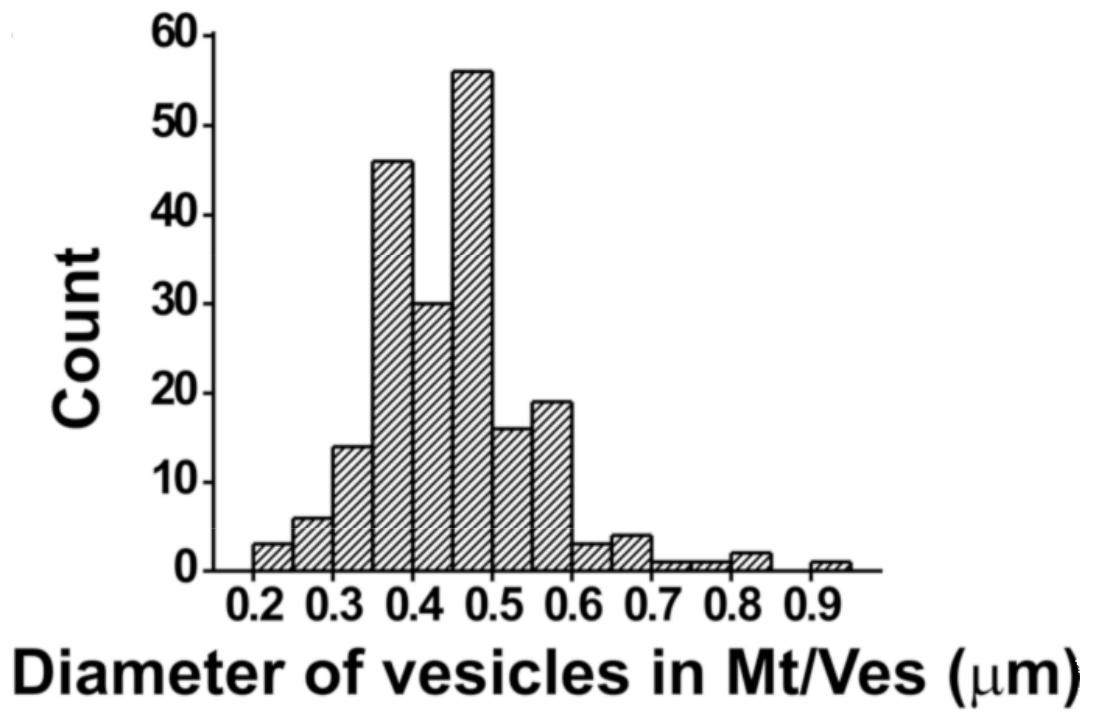

Figure 8. Size distribution of microtubule-associated vesicles isolated from NIH-3T3 cells. The histogram was built as described in Methods.

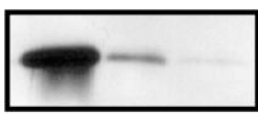

\section{NDPK}

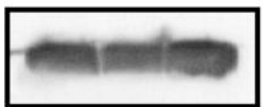

$\beta$-tubulin

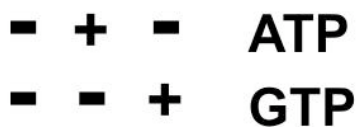

Figure 9. Both ATP and GTP release NDPK from taxolstabilized microtubules and associated vesicles. An extract from resting cells was divided into equal portions that were incubated with taxol in buffer supplemented or not with ATP or GTP (both at $1 \mathrm{mM}$ ); after washes, pellets were processed as described in Methods and tested for NDPK and $\beta$-tubulin. 
sensitive to nucleotides than that of molecular motors, so the nucleotide concentration used in this work is too low to cause elution of significant amounts of dynein and kinesin. For instance, $10 \mathrm{mM}$ ATP releases only $50 \%$ of bound dynein and kinesin from an analogous fraction obtained from rat liver (Oda et al., 1995), with GTP being slightly less effective. Thus, the dissociation of NDPK from the Mt/Ves pellet by nucleotides is not linked to the release of motor proteins and their cargo.

\subsubsection{Characterization of the process leading to NDPK dissociation from microtubule-associated vesicles}

A time course of the release of NDPK from the Mt/Ves by GTP was performed. After the Mt/Ves pellet was washed, $1 \mathrm{mM}$ GTP was added and the samples were incubated at $37^{\circ} \mathrm{C}$ for various times. The Mt/Ves was then re-pelleted and analyzed by SDS-PAGE and immunoblotting. The results show that levels of NDPK in the Mt/Ves pellet are reduced to less than $30 \%$ of that in the control within 1 minute of GTP addition (not shown).

To test whether NDPK can re-bind to GTP-stripped microtubule-associated vesicles, the microtubule/vesicle pellet was incubated with 1mM GTP, washed and thus stripped of NDPK, and then incubated with different concentrations of cytosol. After two washes, pellets were resolved by SDS-PAGE and immunoblotted for NDPK. Analysis of the immunoblots (Fig. 10) shows that not only can NDPK re-bind to GTP-stripped vesicles, but also that the binding is saturable, given that NDPK is abundant in cytosol and is in large excess to the bound fraction.

To determine whether $1 \mathrm{mM}$ GTP dissociated other components concomitantly 


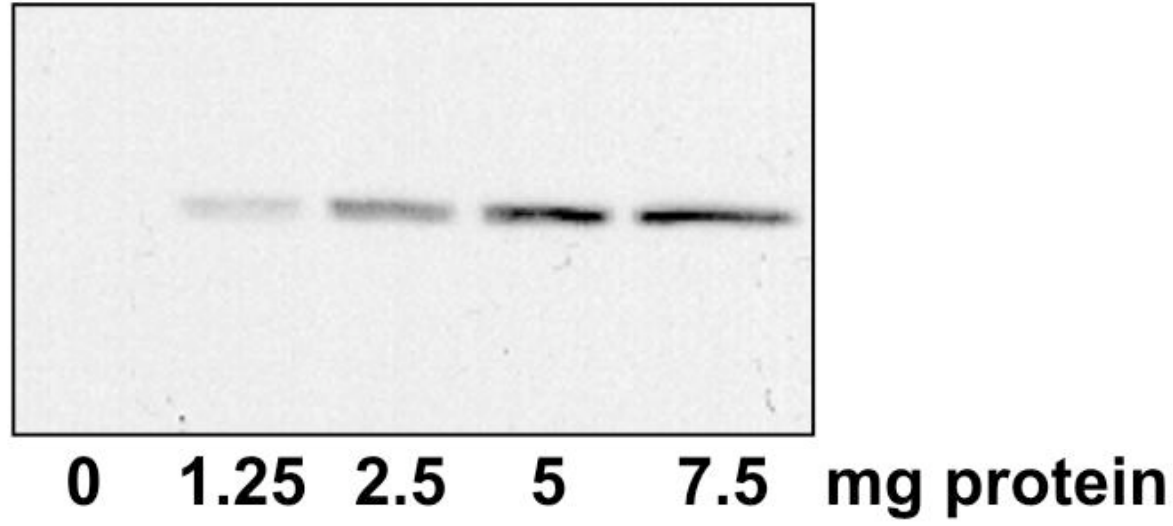

Figure 10. NDPK can re-bind to GTP-stripped pellets, and re-binding is saturable. GTP -stripped pellets were incubated with various amounts of protein and tested for NDPK as described in Methods. Results are representative of $n=3$. 
with NDPK from the Mt/Ves pellet, we washed Mt/Ves pellets in MEPS buffer with or without $1 \mathrm{mM} \mathrm{GTP}$, centrifuged the samples and examined the protein composition of the supernatants by SDS-PAGE followed by silver staining and immunoblotting. A prominent band migrating at the position expected for NDPK $(18 \mathrm{kDa})$ is present in the GTP extract, but not in the control (Fig. 11). Aside from this band, the protein profiles of control and GTP extracts are very similar. Immunoblotting with antibodies specific to NDPKs A, B and C indicated that this band is comprised mostly of NDPK B, which is expressed at higher levels in murine tissues than NDPK A (Barraud et al., 2002); the latter is present in the Mt/Ves fraction and in GTP eluates, but at lower amounts. NDPK C, a potential membrane anchor for NDPK A or B, was not detectable in Mt/Ves (Fig. 12); note, however, that the antibody to NDPK C cross reacts with NDPK A.

To confirm the identity of this protein (arrow, Fig. 11 a) as NDPK and to determine whether the entity eluted by GTP is NDPK alone or a complex of NDPK and other proteins, the supernatants obtained in the presence of GTP were further analyzed by sizeexclusion chromatography, and the NDPK content of individual fractions was measured using enzyme activity assays, SDS-PAGE and immunoblotting (Fig. $11 \mathrm{~b}, \mathrm{c}$ ). As seen in Figure 11b, the GTP extract contains NDPK activity that elutes in one peak centered at $100 \mathrm{kDa}$, the size expected for a hexamer of $18 \mathrm{kDa}$ subunits. Protein staining of gels shows that the enzyme activity overlaps with a protein band migrating at $18 \mathrm{kDa}$ (Fig. 11c), identifiable as NDPK by immunoblotting. Analysis of control extracts shows no NDPK activity above basal levels, no band at $18 \mathrm{kDa}$ and no reactivity with antibodies to NDPK in immunoblots (not shown). These experiments verify that the $18 \mathrm{kDa}$ band seen 
A
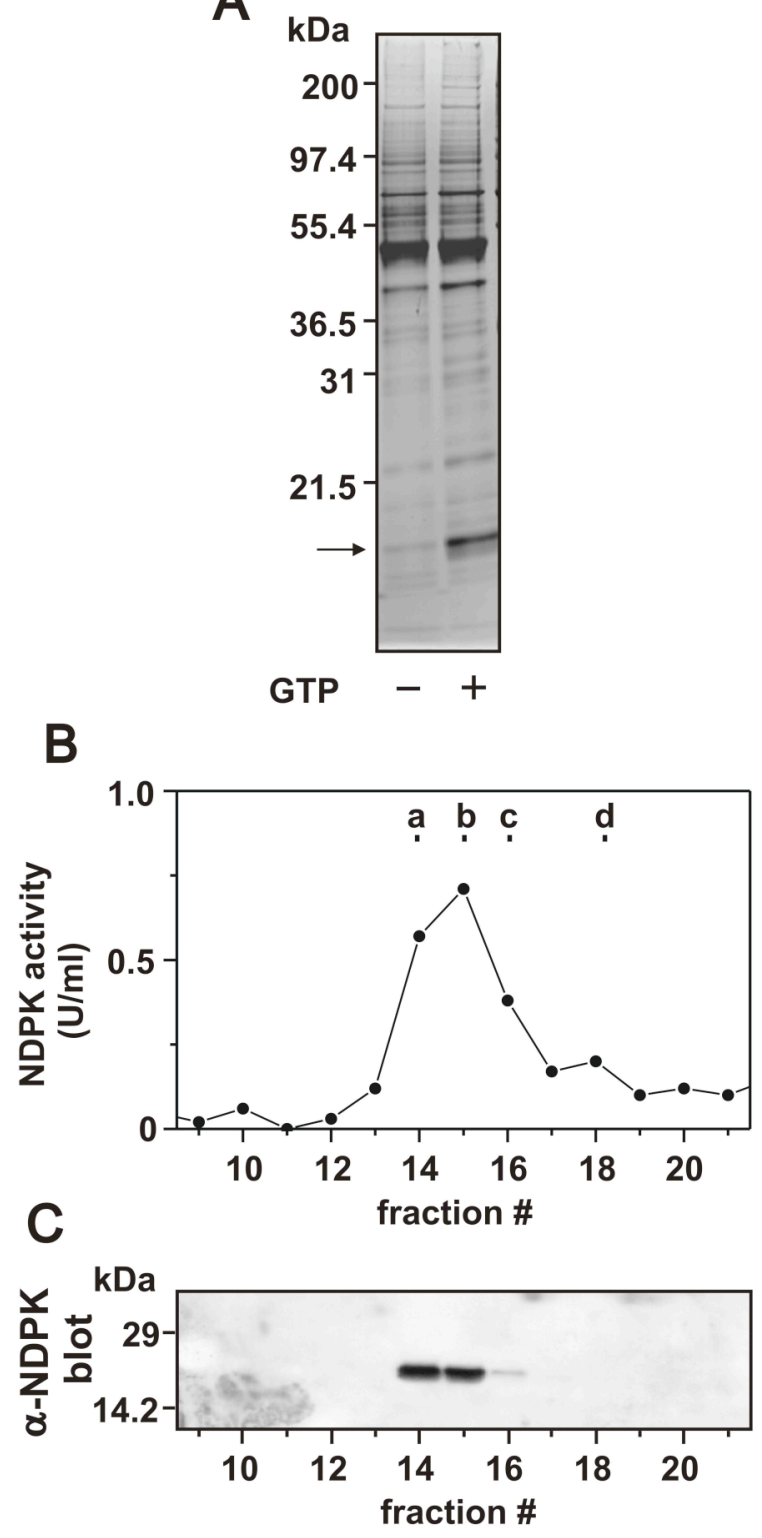

Figure 11. NDPK is released from microtubule-associated vesicles by GTP. Mt/Ves pellets were washed twice with MEPS (containing protease inhibitors and $20 \mu \mathrm{M}$ taxol), and then incubated in the same buffer in the absence or presence of $1 \mathrm{mM}$ GTP. After centrifugation for $30 \mathrm{~min}$ at $4^{\circ} \mathrm{C}$ the supernatants were processed for SDS-PAGE, resolved in a $12 \%$ NuPage gel and stained with silver. B. GTP extract obtained as above, fractionated on Superose 12 as described in Methods. Fractions were assayed for NDPK activity. The elution positions of standards are indicated by (in kDa): a, 158; b, 67; c, 35, and d, 12.4. C. Immunoblot of column fractions from B with antibodies to NDPK. Results shown are representative of 5 independent experiments. 
in Figure 11a is NDPK, and show that GTP releases enzymatically active NDPK, in its native, hexameric state. Moreover, the apparent molecular mass of $100 \mathrm{kDa}$ demonstrates that NDPK is released alone, and not in a complex with other proteins.

We also performed immunoblot analyses of the Mt/Ves pellet with antibodies against putative NDPK binding partners, such as Arf6, dynamin, Rac1, and von HippelLindau protein. Only Rac1 and Arf6 were detected in the Mt/Ves pellet, and neither was removed from the pellet upon nucleotide treatment (not shown). In addition, although $[\alpha-$

$\left.{ }^{32} \mathrm{P}\right]-\mathrm{GTP}$ overlay assays of the Mt/Ves fraction revealed the presence of several small GTP binding proteins, none were released by GTP treatment (Fig.13).

\subsubsection{The binding of NDPK to vesicles is controlled by its catalytic cycle.}

NDPKs bind nucleotides with affinities in the range of 10-200 $\mu \mathrm{M}$, and guanine nucleotides are somewhat preferred over other substrates (Schaertl et al., 1998; Cervoni et al., 2001; Schneider et al., 2002). Therefore, the simplest explanation for the preferential release of NDPK from the Mt/Ves fraction by GTP is that it involves binding of the nucleotide directly to its catalytic site. However, it is also possible that the release of NDPK from the Mt/Ves fraction is secondary to the interaction of GTP with a highly selective binding site, perhaps a GTP-binding protein, and that the effect of ATP is indirect, requiring its conversion into GTP. To distinguish between these possibilities, we examined the effects of the guanine nucleotide analogs guanosine-5'-O-(3thio)triphosphate (GTP $\gamma \mathrm{S})$, guanylyl-imidodiphosphate (GMP-PNP) and guanosine-5'-O(2-thio)diphosphate (GDP $\beta S$ ) on the association of NDPK with the Mt/Ves fraction (Fig. 14a). If release of NDPK by GTP is related to a GTP-binding protein, the activating GTP 


\section{Anti-A}

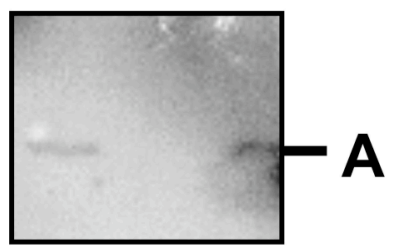

C $\mathbf{G} \mathbf{L}$
Anti-B

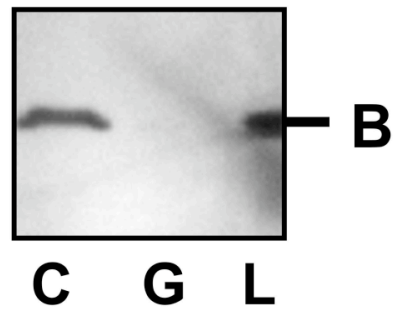

Anti-C

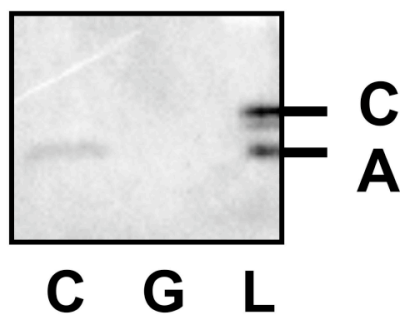

Figure 12. NDPK $A$ and $B$ are present in microtuble-associated vesicles, but NDPK C is not. C - Control (untreated pellets), G - GTP treated pellets, L - NIH3T3 lysate.

\section{$\left[\alpha-{ }^{32} \mathrm{P}\right] \mathrm{GTP}$ overlay of Mt/Ves pellet}

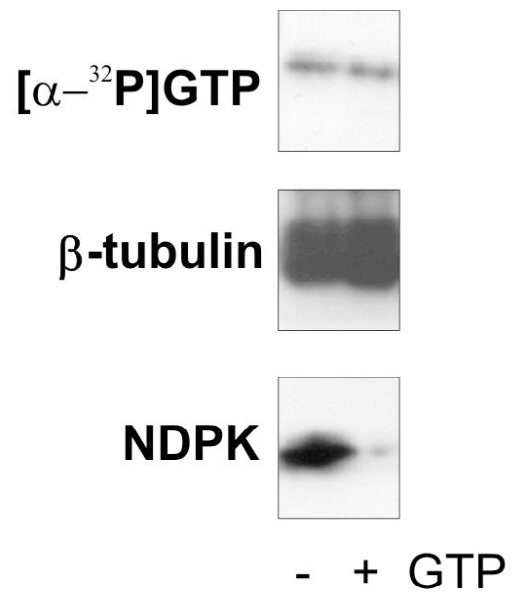

Figure 13. Small GTP binding proteins are not released from microtubuleassociated vesicles by GTP. Untreated and GTP-treated vesicular extracts were resolved by SDS-PAGE and transferred to nitrocellulose. Membrane was incubated with $\left[\alpha-{ }^{32} \mathrm{P}\right] \mathrm{GTP}$ before exposure. 
analogs GTP $\gamma \mathrm{S}$ and GMP-PNP are expected not only to act alike but also to have opposite effects to those of GDP $\beta$, which locks GTP-binding proteins in the inactive form. However, if the release is mediated by the catalytic site of NDPK, the thiophosphate analogs GTP $\gamma \mathrm{S}$ and GDPßS, which are substrates for NDPKs (Schaertl et al., 1998), should behave similarly to GTP. Imidodiphosphate analogs bind to NDPKs with low affinity and are not substrates (Cervoni et al., 2001), so GMP-PNP should have modest effects, if any, on the retention of NDPK by the Mt/Ves fraction. As seen in Figure 14a, $1 \mathrm{mM} \mathrm{GTP} \gamma \mathrm{S}$ and GDP $\beta S$, but not GMP-PNP, are as effective as GTP in eluting NDPK, suggesting that the nucleotide binding site involved is that of NDPK, which implies that the nucleotide effects on the association of NDPK to membranes are intimately linked to its catalytic cycle. To address this issue in depth, we determined the dose-response relationship for release of NDPK from the Mt/Ves fraction for GTP, ATP and ADP. As the concentration of GTP or ATP increases from $1 \mu \mathrm{M}$ to $1 \mathrm{mM}$, the amounts of NDPK bound to the Mt/Ves pellet in the presence of GTP or ATP decreases markedly (Fig. 14b). GTP $\gamma$ S and GDP $\beta$ S also inhibit binding, but with higher potency than ATP or GTP. In contrast, ADP decreases the amount of NDPK bound by $70-80 \%$ at all concentrations tested.

While the results obtained at high concentrations of nucleotides confirm our previous data on the dissociation of NDPK from membranes, examination of the effects of low concentrations of these compounds offered a new insight into the association process. Unexpectedly, the lowest concentration of GTP and ATP, $1 \mu \mathrm{M}$, consistently raised the amount of NDPK associated with $\mathrm{Mt} / \mathrm{Ves}$ to approximately $125 \%$ of the 
A
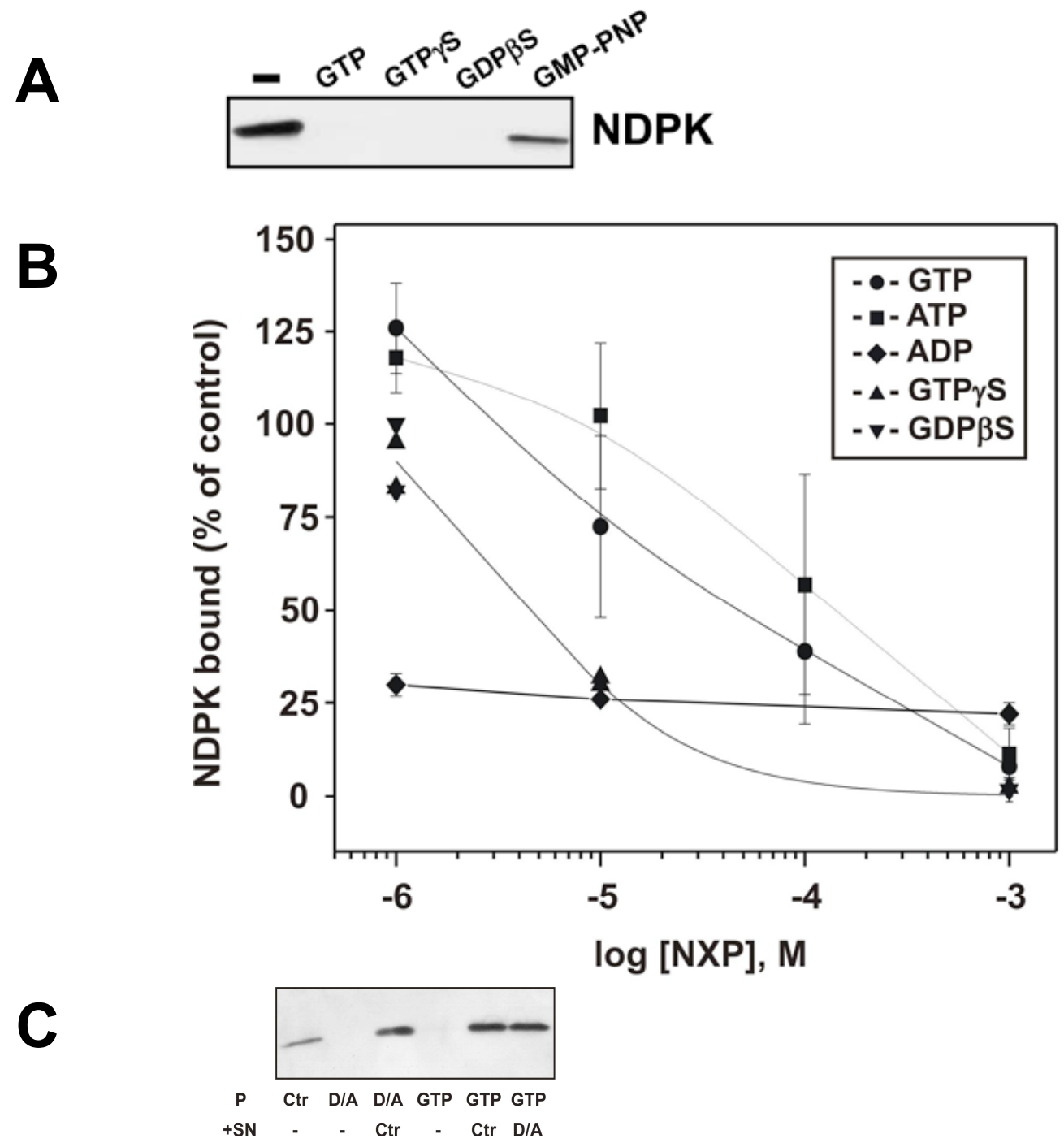

Figure 14. The interaction of NDPK with membranes is controlled by its substrates, and is abolished by ATP depletion. A. Nucleotides were present during taxol-induced polymerization of microtubules at the concentrations indicated, and samples were immunoblotted for NDPK. B. The amounts of NDPK in the resulting MT/Ves pellets were assessed as in Methods. Values shown are ratios of the amounts of NDPK in nucleotide-treated samples to those measured in controls run in parallel with no added nucleotides, expressed as percent of control. $n=2-3$ for GTPYS and GDP $\beta S$, and 3-9 for ATP, GTP and ADP. Error bars indicate S.E. of means. C. Cells were kept in low glucose medium (4 samples) or treated with deoxyglucose and azide (D/A; 2 samples) as in Methods. Following polymerization with taxol and centrifugation, supernatants (Ctr SN, D/A SN) were removed and pellets were washed twice. Three Ctr samples were extracted with $1 \mathrm{mM}$ GTP and pelleted (GTP pellet). Pellets were then resuspended as follows: one each of Ctr, D/A, and GTP pellets in MEPS buffer (-), one each of D/A and GTP pellets in Ctr SN, and one GTP pellet in D/A SN. After $30 \mathrm{~min}$ on ice, samples were centrifuged and the pellets were analyzed by SDS-PAGE followed by immunoblotting with antibodies to NDPK. Results are representative of $n=3$. 
control value (Fig. 14b). This increase is not observed with the GTP analog GTP $\gamma$ S, and suggests that only substrates that transfer phosphate to NDPK and produce the phosphohistidine intermediate $\mathrm{E} \sim \mathrm{P}$ (Scheme 3 ) can promote the association of NDPK with membranes. Indeed, ADP, which is an acceptor for the phosphate of $\mathrm{E} \sim \mathrm{P}$, thereby dephosphorylating the enzyme, only induces dissociation. GDP $\beta S$ and GTP $\gamma$ S, which bind tightly to NDPK but are very poor substrates, act as dose-dependent inhibitors. Collectively, these data indicate that the association of NDPK with Mt/Ves is a reversible process whose dynamics are controlled by the relative amounts of the phosphorylated intermediate, $\mathrm{E} \sim \mathrm{P}$, and the free enzyme, $\mathrm{E}$.

If this reasoning is correct, a reduction in the $[\mathrm{NTP}] /[\mathrm{NDP}]$ ratio in cells, which necessarily decreases the $[\mathrm{E} \sim \mathrm{P}] /[\mathrm{E}]$ ratio, should decrease significantly the amounts of NDPK bound to Mt/Ves. To test this hypothesis, we assessed the levels of NDPK in $\mathrm{Mt} /$ Ves fractions isolated from cells pre-incubated with the glycolysis inhibitor 2deoxyglucose and the mitochondrial poison sodium azide (D/A), a treatment that markedly depletes cytosolic ATP (and consequently other NTPs), and reduces markedly the $[\mathrm{NTP}] /[\mathrm{NDP}]$ ratio (see, for example, Schwoebel et al., 2002). Figure 14c shows that $\mathrm{Mt} /$ Ves pellets prepared from cells pre-treated with D/A contain only traces of NDPK ( $7 \pm 5 \%$ of control, $\mathrm{n}=4$ ), as would be expected if the association of NDPK to Mt/Ves depended on the levels of NTP. There is, however, the possibility that the decrease in binding is due to a secondary effect of the $\mathrm{D} / \mathrm{A}$ treatment, which could decrease the number of NDPK binding sites on the Mt/Ves or induce a decline in the actual amounts of cytosolic NDPK. To address this point, we performed two control experiments: in one, 


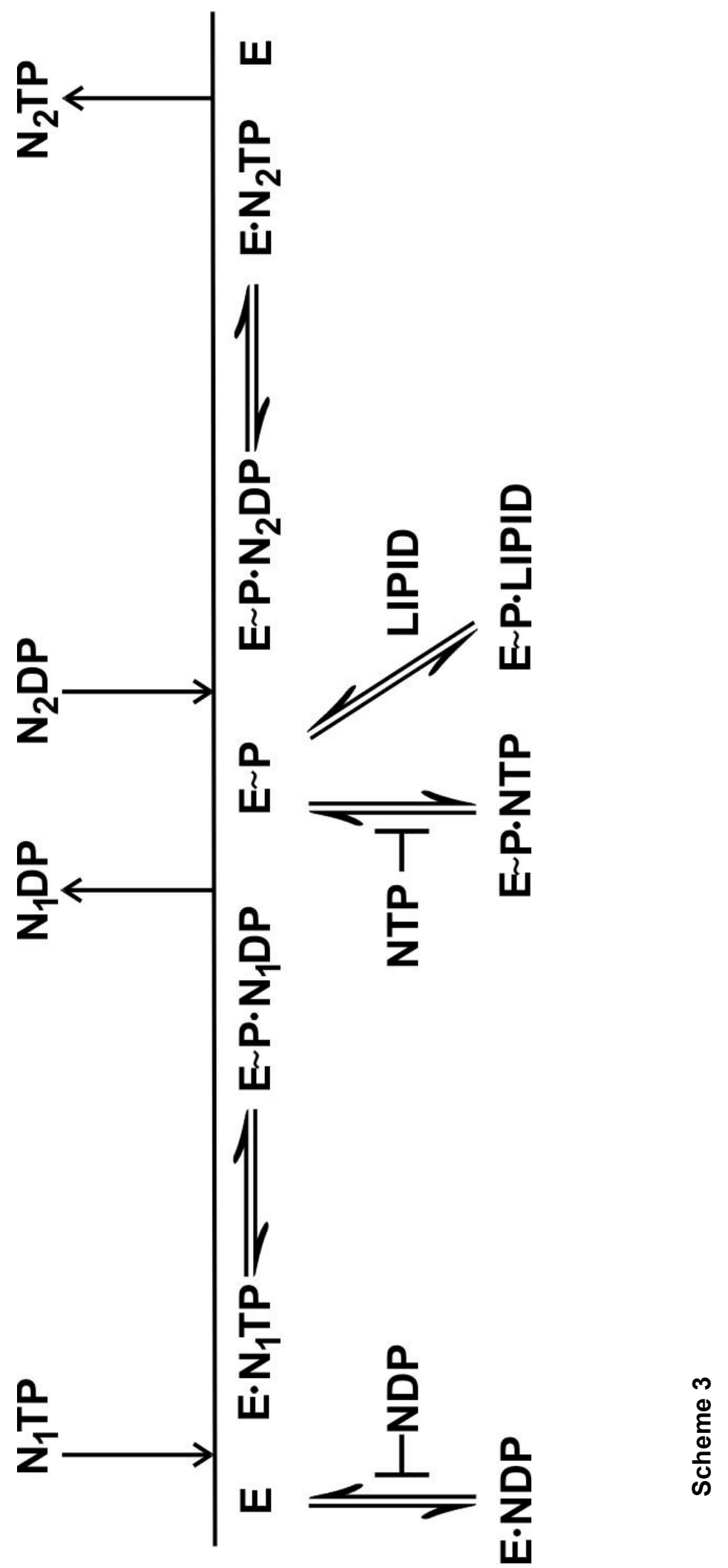


we incubated Mt/Ves pellets obtained from D/A-treated cells with cytosol from the control cells (kept in low glucose medium), centrifuged the preparation and measured NDPK in the pellet. This same procedure was repeated using cytosol from D/A-treated cells or control cells, and Mt/Ves pellets that had been stripped of NDPK with $1 \mathrm{mM}$ GTP (and therefore contain significant amounts of residual GTP). The results (Fig. 14c) show that in all cases NDPK re-bound to the Mt/Ves fraction. This set of experiments indicates that both Mt/Ves membranes and cytosolic NDPK from D/A-treated cells retain their ability to associate, as long as the NTP concentration in the incubation mixture reaches a minimum level. Therefore, the low binding of NDPK to Mt/Ves isolated from cells treated with D/A is likely to be the consequence of a lowered $[\mathrm{NTP}] /[\mathrm{NDP}]$ ratio.

The other possibility is that the effect of D/A treatment is due to lowered GTP levels (as a result of lowered ATP levels). To distinguish whether the result was from lowered GTP or ATP levels, we treated the cells with ribavirin, which depletes cellular GTP levels (Schwoebel et al., 2002). This treatment did not change the amount of NDPK in the pellet (not shown), implying that it is indeed the decrease in the $[\mathrm{ATP}] /[\mathrm{ADP}]$ ratio and not lowered GTP levels that cause the effect of D/A.

\subsubsection{NDPK binds directly to phospholipids}

Inspection of Figure 7 shows that NDPK localizes to nearly all of the vesicular structures visible in the DIC image. Microtubules are involved in the intracellular traffic of membrane vesicles from the secretory, endocytic and recycling pathways, and as a result, Mt/Ves fractions are highly heterogeneous (Oda et al., 1995; Soroka et al., 1999). The widespread distribution of NDPK suggests that it binds to a universal component of 
intracellular membranes, namely lipids. Thus, our next step was to investigate the possibility of direct association of NDPK with lipids.

In preliminary tests we used a protein lipid overlay assay. Membranes strips prespotted with different phospholipids were incubated with cytosol from NIH-3T3 cells, as well as purified NDPKs, and then probed with antibodies against NDPK. Figure 15a illustrates the results obtained with cytosolic NDPK from NIH-3T3 cells and rNDPK-A in the presence and absence of GTP. The cytosolic enzyme associates with PtdIns and PS, whereas rNDPK-A shows a preference for PtdIns(5)P, PtdIns(3)P, PtdIns(4)P and PS. There was no detectable binding of murine or human NDPK to immobilized PC, PE, sphingosine phosphate or lysophospholipids. As with the Mt/Ves experiments, $1 \mathrm{mM}$ GTP reduced the amount of rNDPK-A bound to the phospholipids (Fig. 15b). Although this type of assay does not necessarily reflect affinity or specificity in solution, it is clear from Figure 15 that both murine and human NDPK can bind to pure phospholipids.

We next examined how the binding of NDPK to pure lipids translated into a more physiological experimental system, that is, artificial lipid bilayers, using a sedimentation method (Robbe and Antonny, 2003). Briefly, NDPK from cytosol was incubated with sucrose-loaded small unilamellar vesicles of defined phospholipid composition. The samples were pelleted by ultracentrifugation and the level of NDPK bound to the liposomes was measured as in Methods. Figure 16 shows that cytosolic NDPK from NIH-3T3 bound to liposomes of different compositions, showing a preference for PAcontaining mixes. More importantly, in the presence of 1mM GTP (or ATP), the levels of cytosolic NDPK associated with liposomes were noticeably reduced. Conversely, in the 


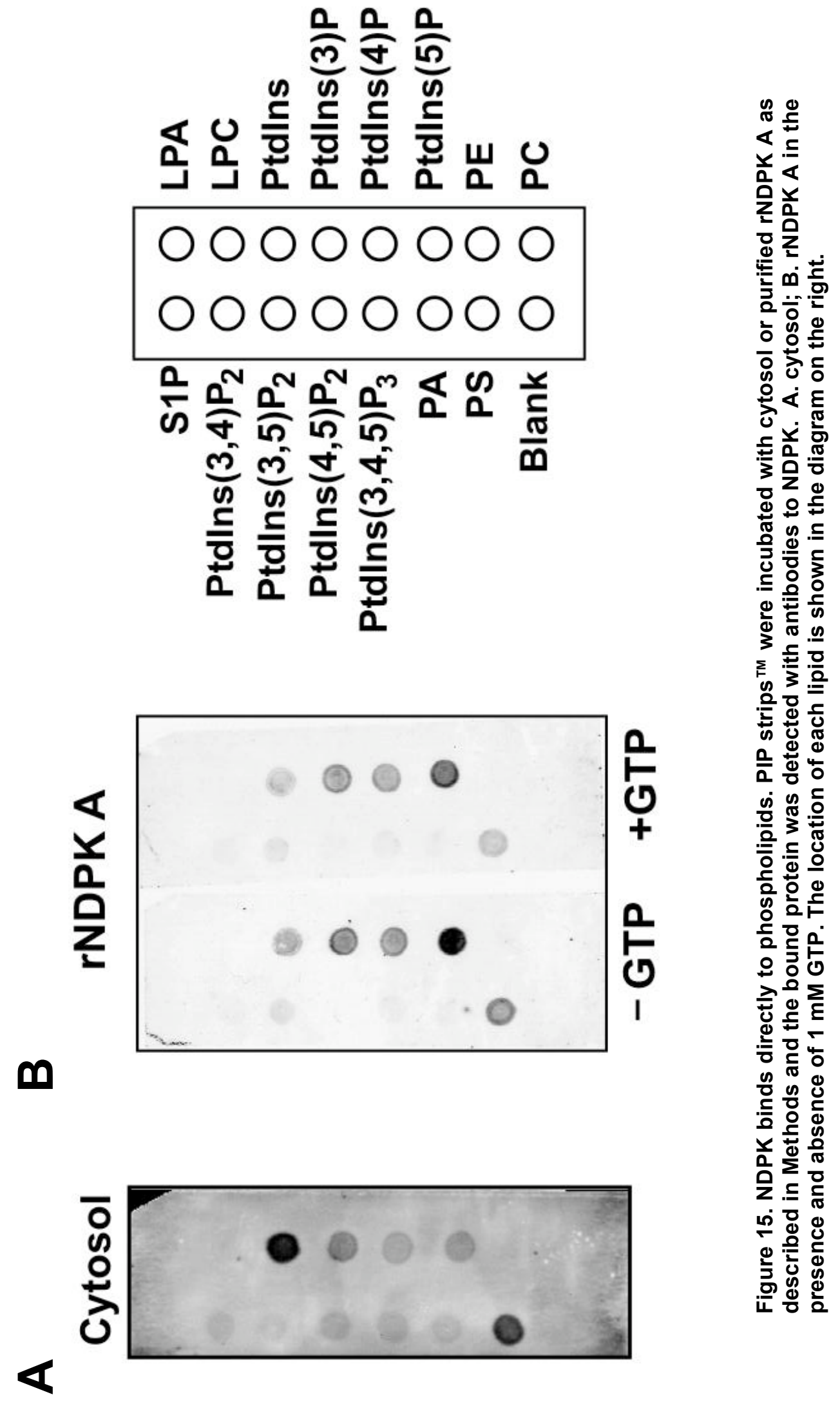




\section{Cytosol (NIH-3T3)}

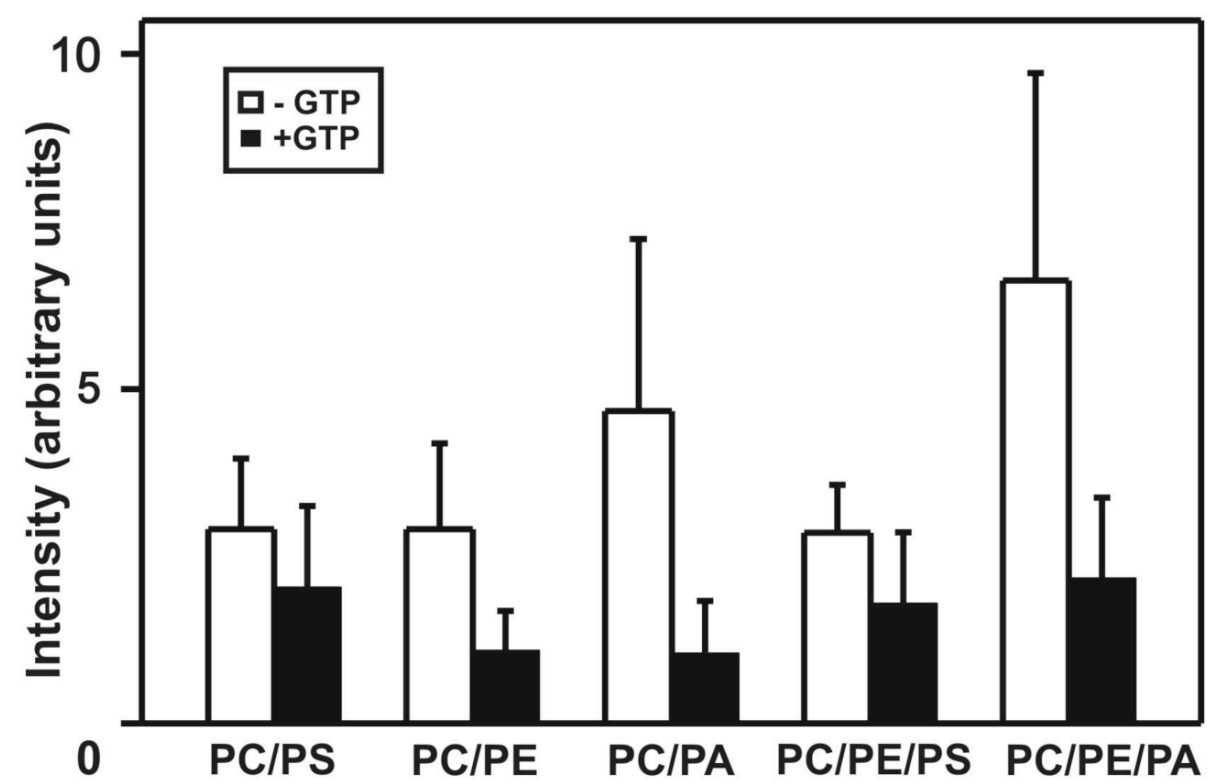

Figure 16. Interaction of NDPK from cytosol with liposomes of defined composition. Liposomes were incubated with cytosol from NIH-3T3 cells as described in Methods. The bound protein was detected by SDS-PAGE and immunoblotting with an anti-NDPK antibody. The bar graph shows NDPK content of pellets treated with $1 \mathrm{mM}$ GTP and untreated pellets. Graph shows the average band intensity measured in 4 experiments, normalized to protein. Error bars are S. E. of means. Although there is no significant difference, there is an obvious trend of preference for PA-containing mixtures. 
presence of $1 \mu \mathrm{M}$ GTP or ATP, binding to liposomes was increased over control by $32 \%$ (PC/PS), $27.5 \%$ (PC/PE), 19.4\% (PC/PA) and 48.1\% (PC/PE/PA), remaining unchanged (97\% of control) for liposomes formed from PC/PE/PS ( $n=3)$.

Similar results were obtained with purified eNDPK (Fig. 17): the purified protein bound to several combinations of phospholipids, and the binding was decreased by $1 \mathrm{mM}$ GTP or ATP. Note that the last step of the purification procedures for both rNDPK A and eNDPK, involve elution of the enzyme from a dye column with ATP; as a result, both preparations are likely to be partially phosphorylated (Robinson et al., 1981) and therefore able to associate with liposomes in the absence of added NTP.

To further characterize the binding of NDPK to liposomes, we prepared liposomes from lipid mixtures that are more representative of intracellular membranes (Table 1). The lipid combinations and proportions in these mixtures are loosely based on the reported compositions (Zambrano et al., 1975; van Meer, 1998; Bremser et al., 1999) of membranes isolated from the endoplasmic reticulum (Mix 1), the Golgi (Mix 2) and plasma membranes (Mix 3). Cytosolic NDPK from NIH-3T3 cells did not discriminate between liposomes made with lipid mixtures 1 and 2, but appeared to prefer mixture 3 over mixture $2(\mathrm{P}<0.05)$. GTP release was observed with the three sets of liposomes, but was more prominent in the case of mixtures 2 and 3 ( $P<0.05$; Fig. 18). Mixture 3 has the highest cholesterol content of all three, and therefore the highest dipole potential (Szabo, 1974). It can be speculated that a higher dipole potential might increase NDPK binding to liposomes by way of electrostatic interactions. 
A

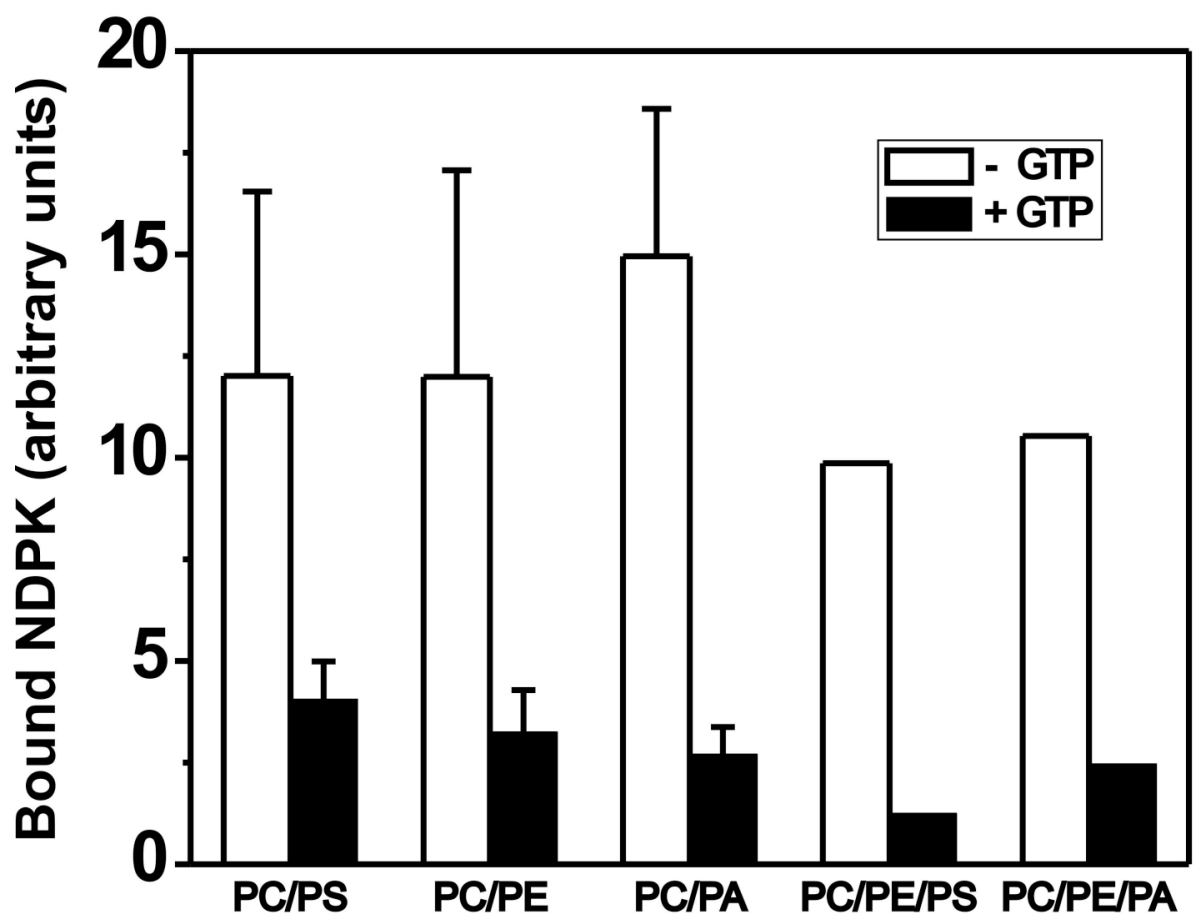

\section{Purified NDPK}

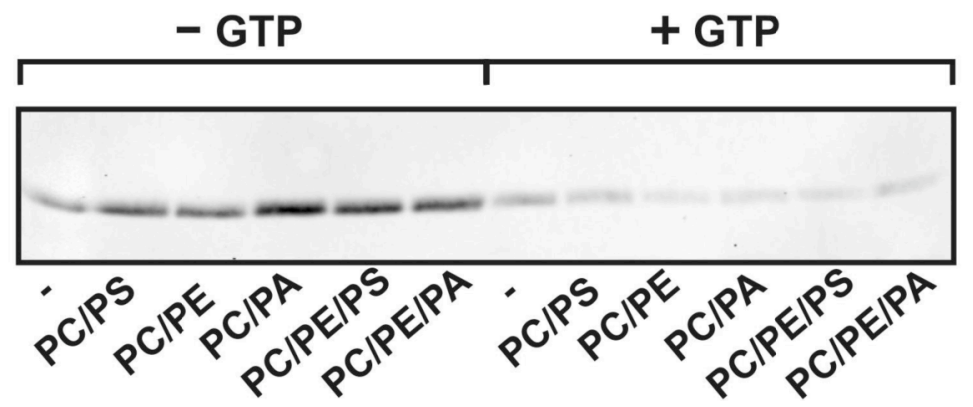

Figure 17. Interaction of purified NDPK from human erythrocytes with liposomes of defined composition. Liposomes were incubated with eNDPK as described in Methods, centrifuged, and washed with buffer alone or buffer containing $1 \mathrm{mM}$ GTP. The bound protein was detected by SDS-PAGE and immunoblotting. The bar graph shows the average band intensity, normalized to protein. A: $n=3$ for mixes of 2 lipids; $n=2$ for mixes of 3 lipids. Error bars are $\mathbf{S}$. E. of means. $p=0.00002$ comparing all Control to all GTP. 


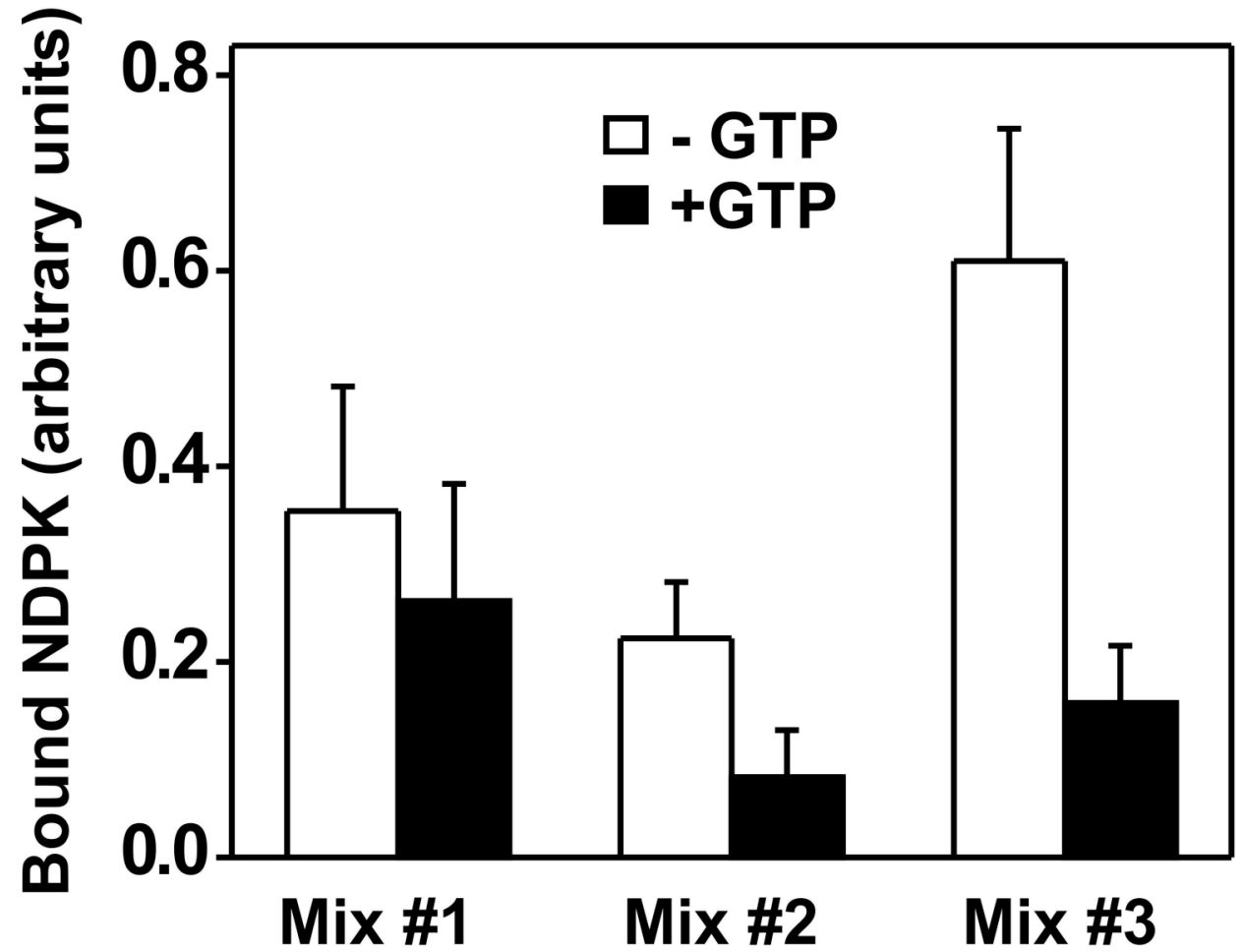

Figure 18. Interaction of cytosolic NDPK with liposomes containing phospholipids, cholesterol and sphingomyelin. Liposomes were incubated with cytosol from NIH-3T3 cells as described in Methods, centrifuged, and washed with buffer alone or buffer containing $1 \mathrm{mM}$ GTP. The bound protein was detected by SDS-PAGE and immunoblotting. The bar graph shows the average band intensity, normalized to protein. Error bars are S. E. of means. Mix 1 vs Mix 2, Mix 1 vs Mix 3, Ctr: $p>0.05$. Mix 2 vs Mix 3, Ctr: $p=0.03$. Mix 1 and 2 Ctr vs GTP: $p>0.05$. Mix 3 Ctr vs GTP: $\mathrm{p}=\mathbf{0 . 0 1 5}$. 
Taken together, these findings imply that the mechanism of binding to the liposomes and to the Mt/Ves isolated from cells is essentially the same. Furthermore, the results shown in Figures 15-18 demonstrate conclusively that the nucleotide-sensitive binding of NDPK to intracellular membranes can be reconstituted with a minimal set of components, namely purified enzyme and lipids.

\subsection{Discussion}

We have used both morphological evidence and cell-free assays to demonstrate a physical association between NDPK, stabilized microtubules and membrane vesicles. The tight binding of NDPK to the Mt/Ves fraction and the full release induced by GTP and other substrates suggest that the interaction is specific. We hypothesize that the dynamic association of NDPK with microtubule-bound vesicles in fibroblasts is relevant to phospholipid synthesis as well as the operation of the multiple GTPases that control intracellular membrane transport. That is to say, in vivo, NDPK might associate with microtubule-bound vesicles when CTP or GTP levels are low, using nucleoside triphosphates such as ATP to phosphorylate CDP or GDP, whereas a rise in CTP or GTP levels leads to its release. Our data agree with studies by other groups implicating NDPK in intracellular vesicle trafficking processes. Thus, NDPK B is a component of isolated phagosomes (Garin et al., 2001) and the Drosophila homolog of NDPK, AWD, regulates dynamin-dependent synaptic vesicle recycling through a mechanism that requires its intrinsic NDPK activity, presumably GTP regeneration (Krishnan et al., 2001). Facilitation of dynamin-based endocytosis by NM23-H1 was also reported for mammalian cells (Palacios et al., 2002). Recently, Baillat et al. (2002) demonstrated a 
direct interaction between NDPK and dynamin I, as well NDPK and phocein, which is homologous to the $\sigma$ subunits of clathrin adaptor subunits, supporting the idea that NDPK plays a role in vesicular traffic.

Our results demonstrate for the first time that soluble NDPKs can associate with membranes by binding directly to lipids. Thus, cytosolic and membrane-bound NDPK are one and the same entity. The interaction does not require additional proteins, although it is conceivable that it might be strengthened by additional interactions with other membrane components. Specifically, the data establish that soluble NDPKs can interact directly with phospholipids, and that the form with highest affinity for lipids is the phosphoenzyme. Therefore, the equilibrium between membrane-bound and soluble NDPK will be determined by the availability of the phosphorylated enzyme.

The equilibrium constant of the reaction,

$$
[\mathrm{E} \sim \mathrm{P}][\mathrm{NDP}] \Omega[\mathrm{E}][\mathrm{NTP}]
$$

is in the range of 0.15 to 0.5 for NDPKs of various species (Garces and Cleland, 1969; reviewed in Lascu and Gonin, 2000). The concentration of ATP in vivo exceeds those of other NTPs, so $[\mathrm{NTP}] /[\mathrm{NDP}]$ is approximately equal to the $[\mathrm{ATP}] /[\mathrm{ADP}]$ ratio, which in most cells, including 3T3 fibroblasts (Takahashi et al., 1994), is around 10. Under these conditions, the ratio $[\mathrm{E} \sim \mathrm{P}] /[\mathrm{E}]$ is $1.5-5$, allowing the binding of NDPK to membranes.

Because of the ping-pong mechanism of NDPK (Scheme 3), NDPs not only dephosphorylate $\mathrm{E} \sim \mathrm{P}$, but also bind to the free enzyme, $\mathrm{E}$, acting as dead end inhibitors (Garces and Cleland, 1969). In either case, if $\mathrm{E} \sim \mathrm{P}$ is the form that associates with membranes, NDP, in this case ADP, should inhibit NDPK or enzyme binding at all concentrations, either by removing free enzyme or by dephosphorylating $\mathrm{E} \sim \mathrm{P}$, and this is 
what we see in Figure 14. In contrast, the $\mathrm{K}_{\mathrm{m}}$ for ATP is $0.3 \mathrm{mM}$, one order of magnitude lower than its $\mathrm{K}_{\mathrm{i}}$ for dead end inhibition, $3.1 \mathrm{mM}$ (Garces and Cleland, 1969). Thus, the binding of NTPs to E P forming abortive complexes (E P NTP) (Scheme 2) is only observed at very high concentrations of NTP and in the absence of significant amounts of NDP. This explains the progressive reduction in the amounts of NDPK associated with membranes as the concentration of ATP (and GTP) is raised.

Two critical questions remain: where does the lipid bind, and how is the catalytic cycle of NDPK coupled to the association and dissociation from membranes? The 3D structures of hexameric NDPK show a symmetrical arrangement that can be described as two identical trimers superimposed horizontally, forming a disk with a diameter of $70 \AA ̊$ and a thickness of $50 \AA$ (Fig. 19; reviewed in Janin et al., 2000). There is a single nucleotide-binding site per subunit, and the top and bottom surfaces of the disk contain three active sites each. The binding site lies in a crevice lined with basic residues, between a loop and a hairpin formed by helices $\alpha \mathrm{A}$ and $\alpha 2$. Preliminary studies using in silico docking (Fig. 20) indicate that this site might accomodate a phospholipid such as PA. In this aspect NDPK is reminiscent of the phosphatidylethanolamine-binding protein (PEBP), a 23-kDa basic protein that binds phospholipids as well as GTP (Serre et al., 1998). Similarly to NDPK, in the crystal structure of PEBP the ligand binding site lies in a narrow crevice near the surface, proximal to a strip of basic residues. Like NDPK, the bovine PEBP structure is not related to other nucleotide binding proteins, or to known lipid-binding proteins.

There are no detectable differences between the $3 \mathrm{D}$ structures of the 


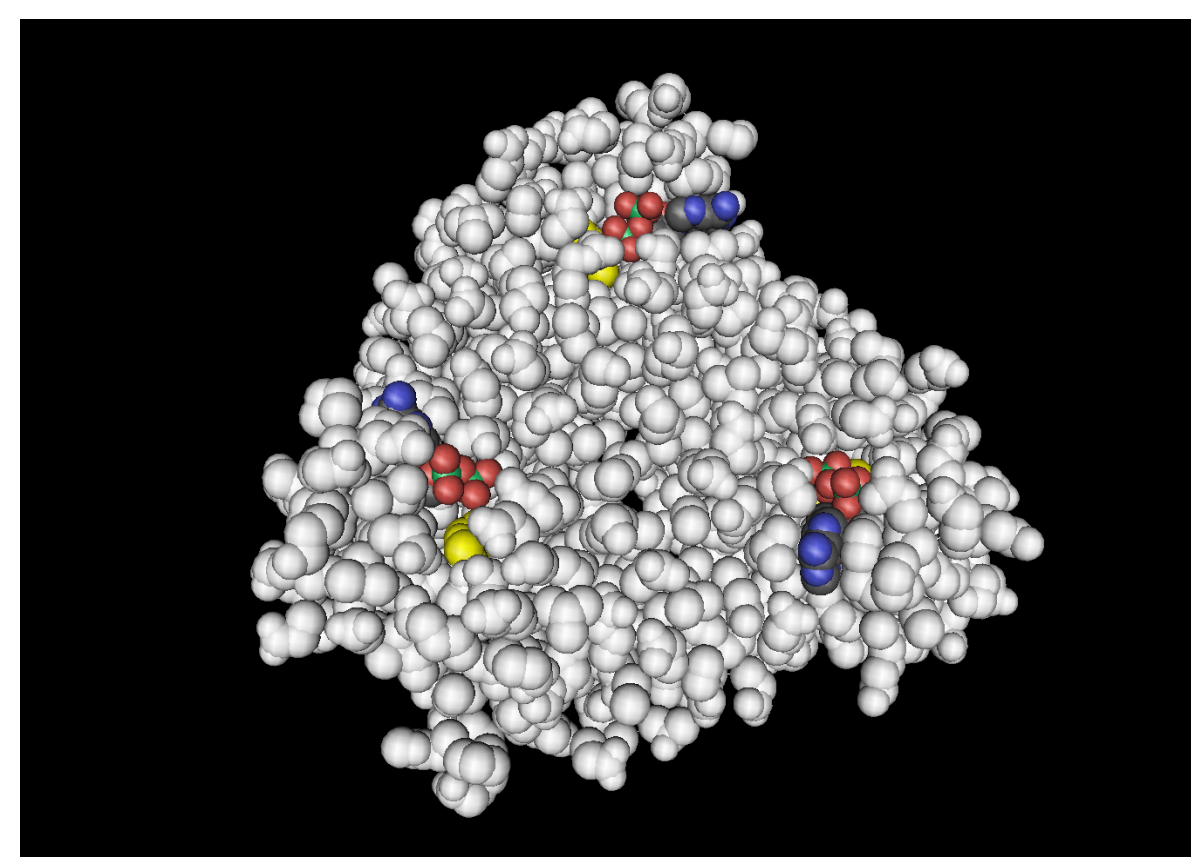

Figure 19. Space-filled model of NDPK with ADP bound to active site. View is of bottom of trimer. White: NDPK; Yellow: Histidine in active site; Blue/Red: ADP.

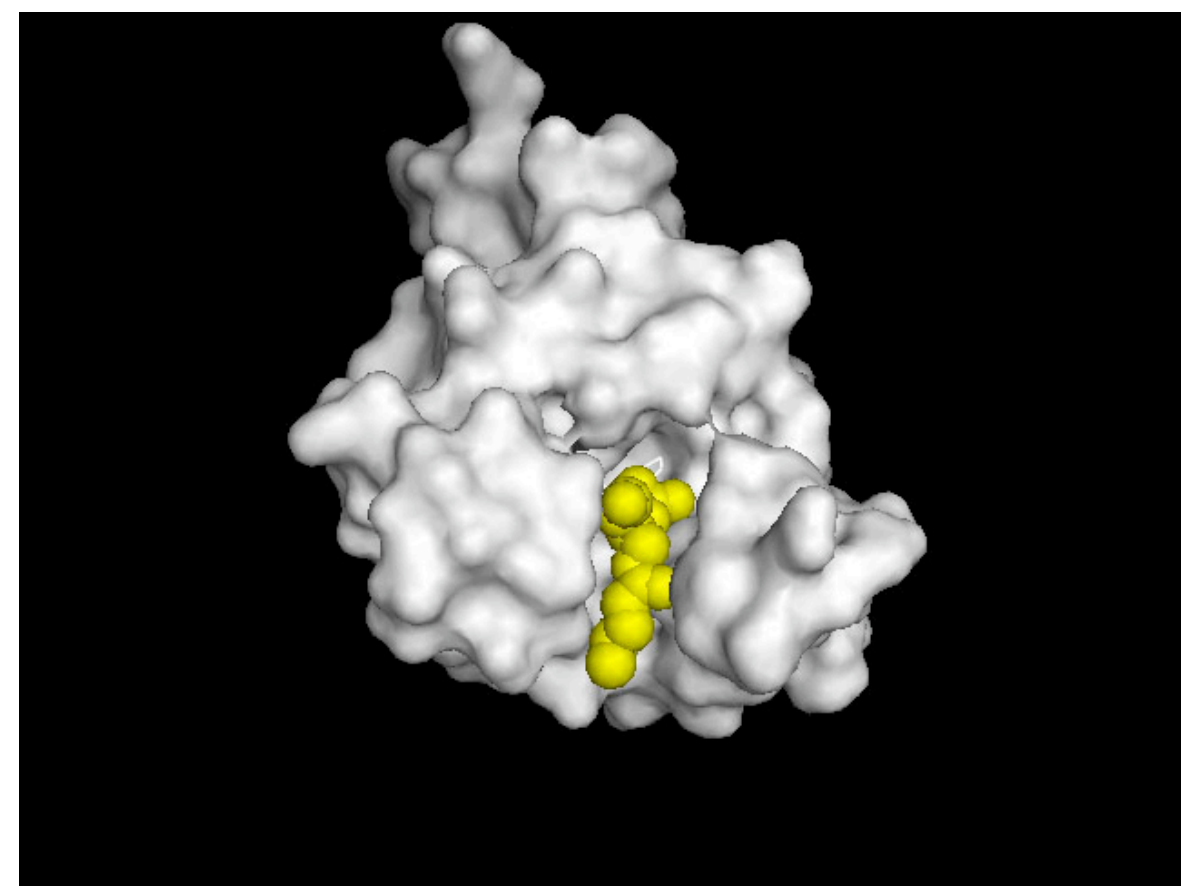

Figure 20. In silico docking of phosphatidic acid into the nucleotide binding site of an NDPK monomer. 
phosphorylated NDPK and the free enzyme (Morera et al., 1995), but binding of nucleotides to the active site results in a small conformational change, where the $\alpha \mathrm{A}-\alpha 2$ hairpin moves $2 \AA$ and locks the nucleotide in place. This suggests a mechanism by which the conformational change associated with nucleotide binding could trigger dissociation of NDPK from bilayers: in E P, the nucleotide binding site is in the "open" conformation, allowing its binding to phospholipids, while occupation of the site, followed or not by phosphate transfer to NDP, closes the cleft and releases the enzyme from the lipid. Soluble NDPK is eventually rephosphorylated by NTP, and is again capable of associating with membrane lipids.

Our data are in agreement with results from other laboratories showing that NDPKs can interact with lipids. Kikkawa et al. (1992) found that soybean phospholipids had a stimulatory effect on NDPK activity, and recently Epand et al. (2007) demonstrated that mitochondrial NDPK-D (or NM23-H4) cross-links bilayers and transfers lipids between them. Note, however, that NDPK-D only transfers lipids between liposomes containing $30 \% \mathrm{~mol} / \mathrm{mol}$ of cardiolipin, and the process is observed in the absence of added $\mathrm{Mg}^{2+}$ and nucleotides. The differences in behavior between cytosolic and mitochondrial NDPK isoforms are not surprising; although NDPK-D is structurally quite similar to NDPKs A and B, it is a very basic protein (with a $\mathrm{pI}$ of 10.3 , whereas A and B have PIs of 5.8 and 8.5 , respectively) and its sequence diverges significantly from those of the A and B isoforms.

In conclusion, our present studies suggest that in vivo the high $[\mathrm{ATP}] /[\mathrm{ADP}]$ ratio promotes phosphorylation of NDPK and its association with membranes, where it could 
transfer phosphate to locally generated NDPs. Schneider et al. (2002) suggested that because of its high concentration in the cytosol (Parks and Agarwal, 1973) and the expected prevalence of its phosphorylated state at normal [ATP]/[ADP] ratios, soluble NDPKs could serve as a cellular reservoir of high energy phosphate. Targeting of such a reservoir to membranes may be relevant to the operation of the pathways of phospholipid synthesis, which require CTP (reviewed in Voelker et al., 2000), as well as the multiple GTPases that control intracellular membrane transport. 


\section{CHAPTER 4: NDPK Moves into the Developing Primary Cilium}

\subsection{Abstract}

Immunofluorescence staining of murine NIH-3T3 fibroblasts grown at high density shows that cytosolic NDPKs A and B localize to a sensory organelle, the primary cilium. Similar results are obtained with Xenopus A6 and Madin-Darby canine kidney (MDCK) kidney epithelial cells, suggesting that NDPKs are a universal component of the primary cilium. The translocation of NDPK into primary cilia of A6 cells depends on size, taking place only when cilia reach a critical length of 5-6 $\mu \mathrm{m}$. In mature cilia, NDPKs are distributed along the ciliary shaft in a punctate pattern that is distinct from the continuous staining observed with acetylated $\alpha$-tubulin, a ciliary marker and axonemal component. We developed the first method for the isolation of a fraction enriched in primary cilia. This allowed us to determine that ciliary NDPK is enzymatically active, and is associated with the membrane and the matrix, but not the axoneme. In contrast, acetylated $\alpha$-tubulin is found in the axoneme and, to a lesser extent, in the membrane. Based on the tightly regulated translocation process and the subciliary distribution pattern of NDPK, we propose that it plays a role in the elongation and maintenance of primary cilia through its ability to regenerate the GTP utilized by axonemal microtubule turnover and transmembrane signaling.

\subsection{Introduction}

Members of the NM23/NDPK family are frequently found in association with microtubular structures (Nickerson and Wells, 1978; Jacobs and Huitorel, 1979; Huitorel

et al., 1984; Pinon et al., 1999). Thus, although NDPKs A and B do not bind directly to 
purified tubulin (Islam and Burns, 1985; Melki et al., 1992), these proteins co-localize with microtubules during interphase (Pinon et al., 1999), and bind to membrane vesicles associated with microtubules (Gallagher et al., 2003; Mitchell et al., submitted). NDPK A is a component of the centrosome and co-immunoprecipitates with $\gamma$-tubulin (Roymans et al., 2000), and NDPK is one of the nucleotide metabolizing enzymes found in Chlamydomonas flagella and Tetrahymena cilia (Watanabe and Flavin, 1976). NDPKs A and B, as well as NM23-5, localize to flagella of spermatozoa (Munier et al., 2003), and NM23-5 and 7 are associated with the isolated axoneme of human respiratory cilia (Ostrowski et al., 2002). NM23-8 is located in the fibrous sheath, a cytoskeletal structure that envelops the sperm axoneme (Miranda-Vizuete et al., 2003), and is also expressed in the respiratory tract (Duriez et al., 2007). NM23-9 is present in axonema from cilia and spermatids, and one of its splice variants binds directly to microtubules (Sadek et al., 2003).

During our studies of the intracellular localization of NDPKs A and B, we found that these proteins localize to a microtubule-based structure called the primary cilium. Primary cilia are hair-like projections originating from the mother centriole present in most vertebrate cells, whose membrane and internal composition differ from those of the cell (for reviews see Poole et al., 1985; Wheatley et al., 1996; Pazour and Witman, 2003; Praetorius and Spring, 2005; Satir and Christensen, 2007; Satir and Christensen, 2008). Although discovered over a century ago (Zimmerman, 1898), other than structural characteristics and prevalence, little was known about primary cilia until fairly recently. The internal structure of primary cilia differs noticeably from that of motile cilia and 
flagella: whereas the latter's axoneme is composed of nine peripheral doublet microtubules surrounding two singlet microtubules $(9+2$ arrangement), primary cilia do not have the core doublet and thus are called "9+0" cilia. Primary cilia also lack other structures linked to motility, such as dynein arms, nexin links and radial spokes, and are thus considered, with the exception of the cilia of the node (or embryonic organizer) of gastrula stage embryos, to be immotile.

Primary cilia were at first thought to be vestigial appendages devoid of function. Subsequent studies (e.g. Gallagher, 1980) noted their widespread presence and conserved ultrastructure, suggesting that these organelles may have an important function in cells. Some hypothesized that primary cilia might have a sensory role (see Poole et al., 1985 and references thereof), but proof of this function was missing until the last several years. Clear-cut evidence came from the work of Praetorius and Spring $(2001 ; 2003)$ who demonstrated that primary cilia act as flow sensors in renal cells, responding to bending with increases in intracellular calcium. Now, work from several laboratories upholds the idea that primary cilia are sensory organelles (reviewed in Pazour and Witman, 2003). Notably, mutations that induce defects in primary cilia are connected with a series of pathological conditions, among these renal cystic disease, situs inversus, and retinitis pigmentosa (Pazour and Rosenbaum, 2002; Sloboda, 2002; Luo et al., 2003; Davenport and Yoder, 2005). Recently, Duriez et al. (2007) identified defects in the gene encoding NM23-H8, including a truncation that removes the active site region of the first NDPK domain and the entire second NDPK domain, in patients with primary ciliary dyskinesia (Duriez et al., 2007). Other mutations that affect the formation and/or structure of 
primary cilia lead to liver and pancreas problems and developmental abnormalities (reviewed in Michaud and Yoder, 2006). Loss of primary cilia after development has been shown to lead to hyperphagia and obesity, in a syndrome similar to that caused by loss of neurons of the pro-opiomelanocortin (POMC) region of the brain (Davenport et al., 2007).

A number of studies have shown that in several cell types elements of signaling pathways such as the polycystins and GPCRs for serotonin $\left(5-\mathrm{HT}_{6}\right)$ and somatostatin (sst3), PDGF receptor (PDGFR)- $\alpha \alpha$ and hedgehog (Hh) signaling localize specifically to the membrane of primary cilia (Brailov et al., 2000; Schulz et al, 2000; Pazour et al., 2002; Yoder et al., 2002; Corbit et al, 2005; Schneider et al., 2005). Indeed, many of the diseases cited above arise from signaling defects in the primary cilia (Christensen et al., 2007). For this reason, the primary cilium has been aptly described as the cell's antenna (Pazour and Witman, 2003) which initiates responses to stimuli that control differentiation, development and proliferation. Because NDPKs have been reported to affect all the latter processes by unknown mechanisms (reviewed in Otero, 2000; Kimura et al., 2000; Arnaud-Dabernat et al., 2003), our results raise the possibility that the clue to some of these cellular functions of cytosolic NDPKs is their localization in primary cilia.

\subsection{Results}

\subsubsection{NDPK is present in primary cilia of NIH-3T3 fibroblasts}

Labeling of sub-confluent, serum-deprived NIH-3T3 fibroblasts with an antibody to a conserved region of NDPKs $\mathrm{A}$ and $\mathrm{B}, \mathrm{Ab}-1$, produces a strong signal in the nucleus and in the cytoplasm, as previously described (Gallagher et al., 2003). However, in cells 
grown at high density, Ab-1 labels an additional, rod-like organelle that varies in length from 1-5 $\mu \mathrm{m}$. This structure is found near the nucleus of virtually all cells in the specimen (Fig. 21), and has the morphology of a primary cilium. Primary cilia contain stable microtubules that are enriched in a post-translationally modified form of tubulin, acetylated $\alpha$-tubulin (Piperno et al., 1985; Piperno et al., 1987). When we labeled cells simultaneously with $\mathrm{Ab}-1$ and antibodies to acetylated $\alpha$-tubulin we observed overlap of the two labels in this structure (Fig. 21, merged). In addition, the antibody to NDPK labels the nucleus, while acetylated $\alpha$-tubulin is present in some cytoplasmic microtubules. However, as seen in the merged image (Fig. 21) and in the insets, the colocalization of the two antigens is limited to the primary cilia, whose dual labeling is sufficiently well defined to be readily visible in nearly all cells. These results indicate that NDPKs A and B are components of primary cilia of murine fibroblasts. Nevertheless, the cilia in these cells are diminutive, and as a result the details of the distribution of NDPKs are difficult to discern. In contrast, other cell types such as kidney epithelial lines form polarized monolayers with apical primary cilia that are remarkably long. Therefore, we carried out the next set of experiments in the A6 cell line derived from the distal nephron of Xenopus laevis, using affinity-purified antibodies to amphibian NDPK to detect its localization.

\subsubsection{NDPK localizes to primary cilia of A6 kidney epithelial cells}

Localization of acetylated $\alpha$-tubulin and NDPK was examined in A6 cells cultured in complete medium for 7-9 days post-confluence. Under these conditions the incidence of primary cilia is high, and they are far longer than those seen in NIH-3T3 

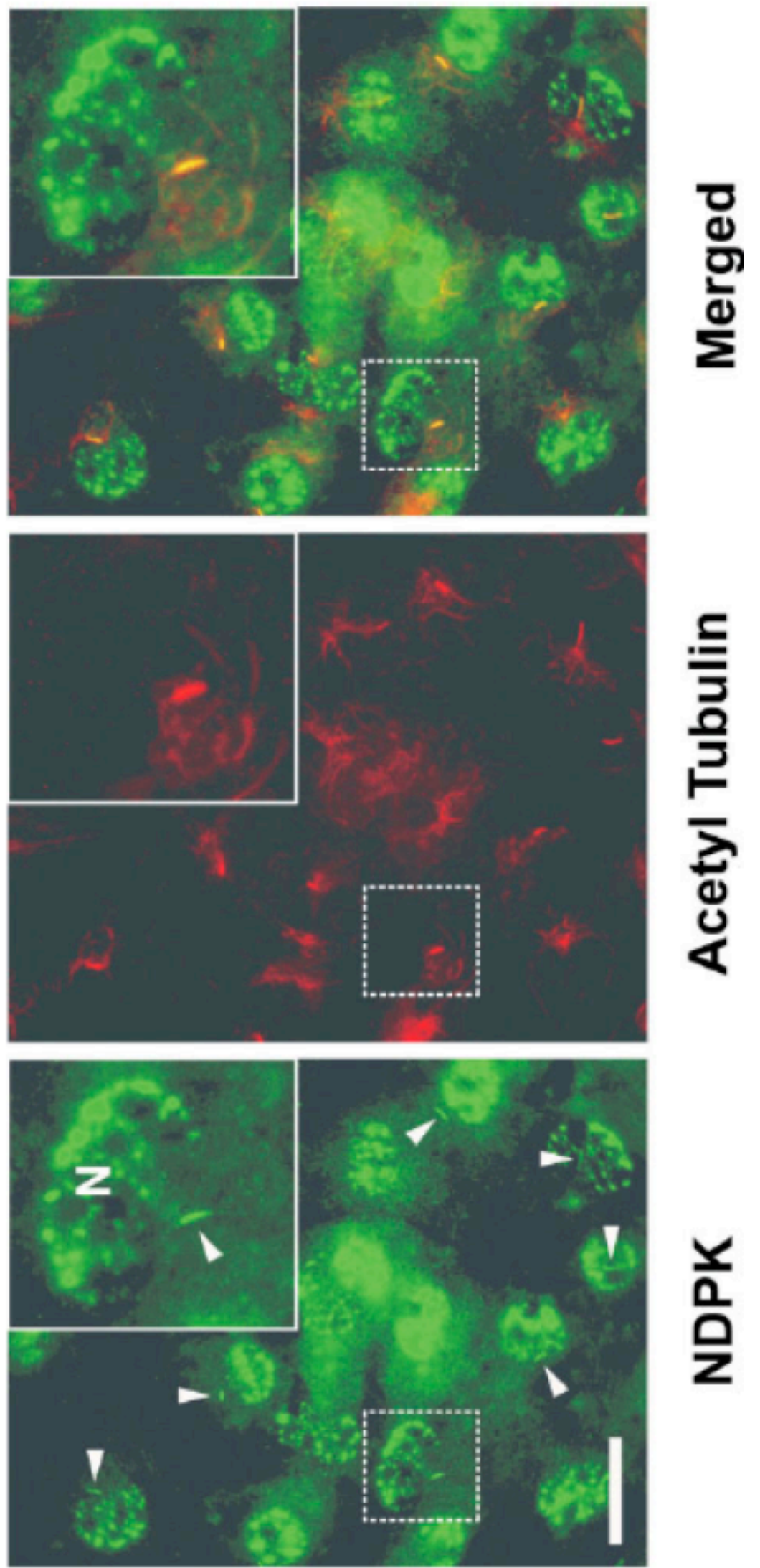

을

ఏ 紊

응 $\frac{2}{\sqrt{0}}$

을

응

군

웡 的

है

ฏ

⿰

논

는

등

ํํㅇ

ज 잉

मึ

क⿺

응

을 훙

粮

$m$ ำ

꼬 음

之婛

드 ㄷํㅇ

ह

o

त्ञ

로

등 들

들 트

유 순

ต 8

항

Nㅡㄴ

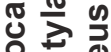
으엉

ำ

之 을 z

亡

는믄

는믈

흔은 는 
cells. Our measurements show that in A6 cells cilia can extend up to $50 \mu \mathrm{m}$ from the apical surface, with the average length being $23.4 \mu \mathrm{m}(\mathrm{n}=53)$. In flow-fixed preparations (Fig. 22, a,b) NDPK and acetylated $\alpha$-tubulin co-localize along the shaft of the cilia; overlap is also seen in swellings at the tip of cilia (see below). To determine whether the co-localization of acetylated $\alpha$-tubulin and NDPK in renal epithelial cells is indeed restricted to the primary cilium as seen in $3 \mathrm{~T} 3$ cells, confluent A6 cells already expressing cilia were lifted gently, re-plated at lower density and allowed to adhere for 2 hours before staining. This approach reduces cell density without removing all primary cilia, allowing examination of the labeling pattern of individual cells. Figure $22(\mathrm{c}, \mathrm{f})$ shows clear overlap of the two labels in the primary cilium. Together with the data from NIH-3T3 cells, these results demonstrate that NDPK is a regular component of mature primary cilia.

In images taken at higher magnification, a distinctive feature of the distribution of NDPK in cilia becomes evident: unlike acetylated $\alpha$-tubulin (Fig. 23a), NDPK is not evenly distributed inside the cilium. Rather, it is present in discrete spots distributed in an irregular pattern along the length of the cilia (Fig. 23b). Thus, the co-localization with acetylated $\alpha$-tubulin is not complete, suggesting that NDPK is not a component of the axoneme. Also, these images show that NDPK is concentrated in the cilium, but not at the area around its base. This localization pattern is similar to those reported for proteins linked to renal kidney disease, cystin (Hou et al., 2002) and inversin (Morgan et al., 2002).

\subsubsection{NDPK distribution during primary cilium development in A6 cells.}



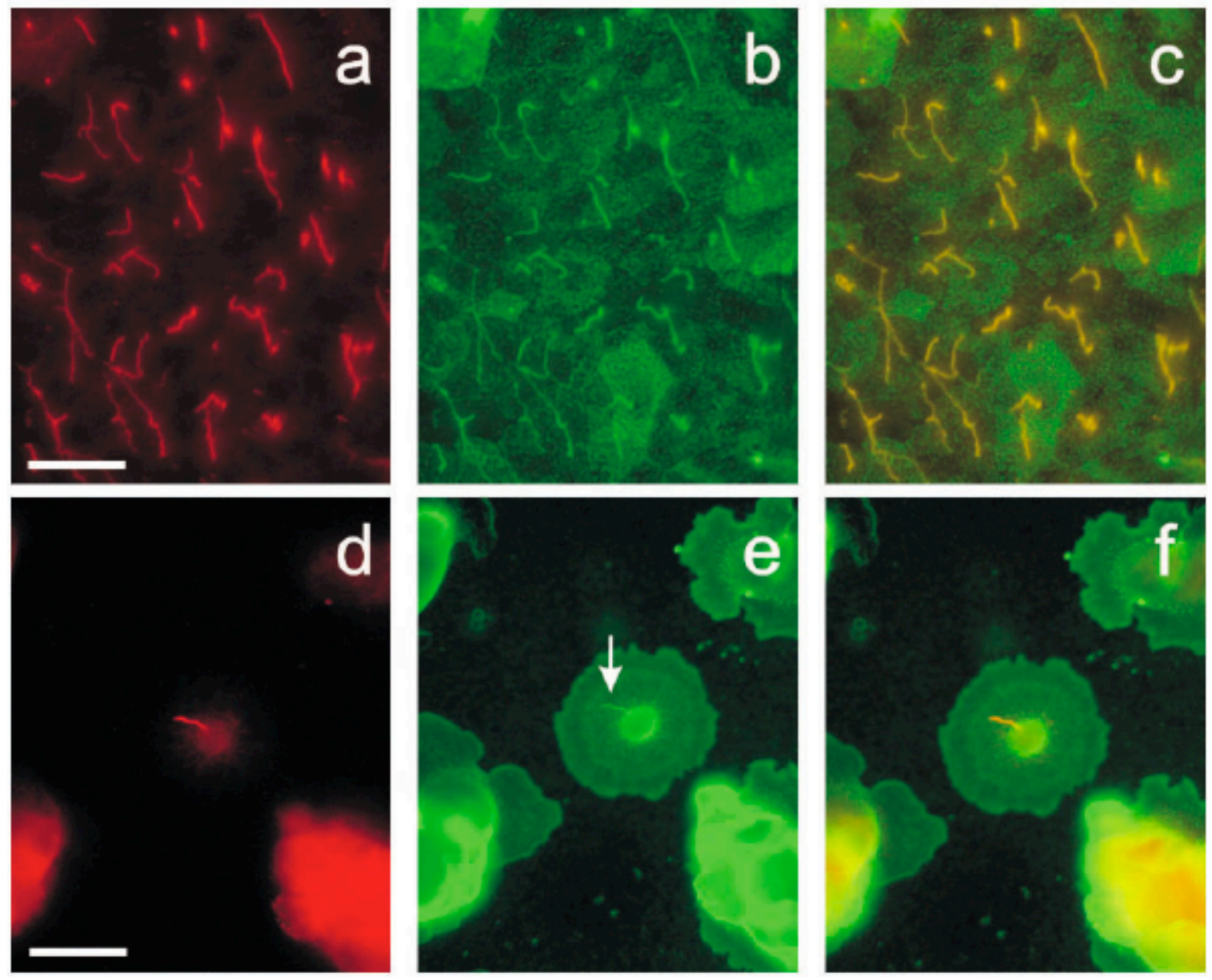

Figure 22. Localization of NDPK in primary cilia of A6 kidney epithelial cells. A6 cells were grown in full medium for 7 days post-confluence and either fixed and stained directly $(a, b)$, or lifted, replated at a lower density, and stained after $2 \mathrm{~h}(\mathrm{c}, \mathrm{d})$. Acetylated $\alpha$-tubulin (a,c); NDPK(b,d). Arrow in d shows primary cilium. Bars: $20 \mu \mathrm{m}$. 
Our next objective was to determine whether NDPK is present throughout primary cilia development. To this end, we lifted subconfluent A6 cells that were not yet expressing cilia, plated them at high density, and stained the specimens at intervals with antibodies to acetylated $\alpha$-tubulin and to NDPK. Primary cilia were absent from spreading cells, and became evident only when cells were 70-80\% confluent (Fig. 24a). At this stage just a few cells had cilia; some of these structures were rather short and did not stain for NDPK (Fig. 24a, b, c, arrowhead), in contrast to the longer ones (Fig. 24b, arrow). At $90-95 \%$ confluence cells were still dividing (see mitotic spindle in 24d), but the length and incidence of primary cilia had increased considerably, and all contained NDPK (Fig. 24e). Confluent cells fixed 9 days after plating expressed very long cilia (Fig. 24g) that stained strongly for NDPK (Fig. 24h). Surprisingly, analysis of the data by measuring cilia and determining whether they are positive or negative for NDPK shows that the translocation of this protein into primary cilia is abrupt, taking place when cilia reach 5-6 $\mu \mathrm{m}$ (Fig. 25). This observation indicates that NDPK is not involved in the early processes of primary cilia assembly, but becomes a part of these organelles as they mature and elongate, and that transport of NDPK into cilia occurs at a definite stage of cilium elongation. This suggests in turn that entry of NDPK into the cilium is a tightly regulated event. In a previous example of regulated movement of a protein into flagella, Aurora A kinase was rapidly translocated into Chlamydomonas flagella in response to rises in cAMP (Pan and Snell, 2000). However, in contrast to NDPK, which appears during cilium formation, Aurora $\mathrm{A}$ is recruited to mature, pre-existing flagella.

\subsubsection{NDPK is present in isolated primary cilia.}



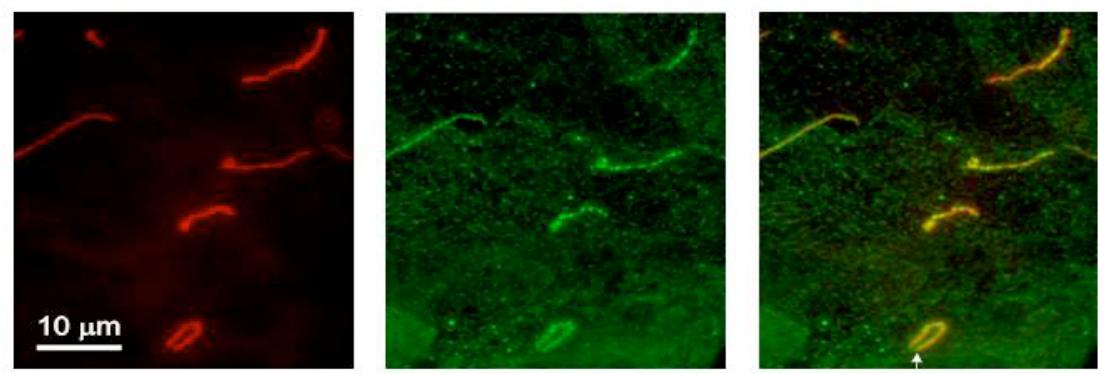

Figure 23. NDPK stains primary cilia in a punctate pattern. A6 cells grown in full medium for 7 days post confluence were fixed, stained with antibodies to acetylated $\alpha$-tubulin (Left) and NDPK (Center) and photographed at high magnification (100X objective). Merge, right panel.
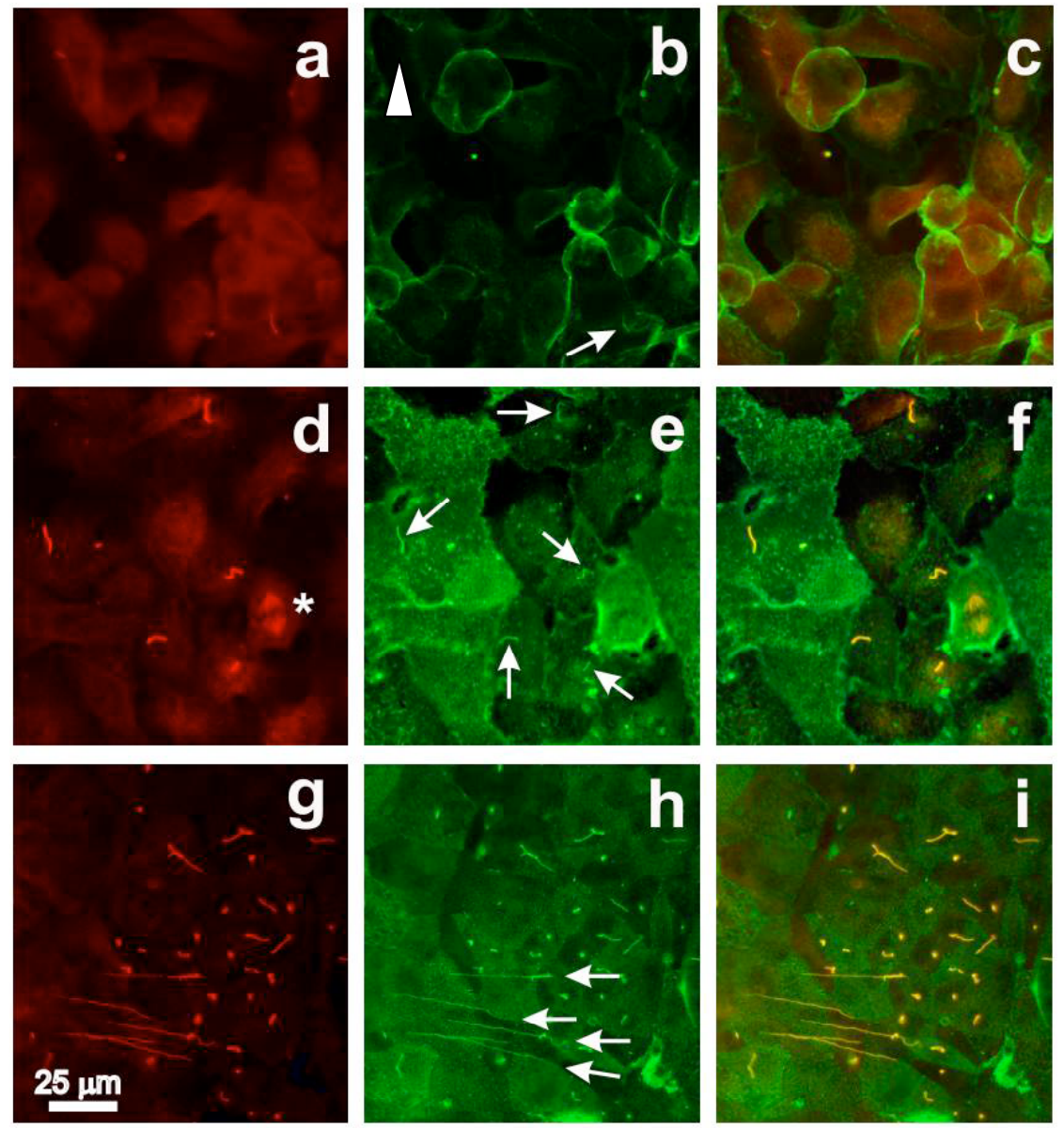

Figure 24. Localization of NDPK during primary cilium development. Subconfluent A6 cells not yet expressing primary cilia were plated at high density and stained at intervals of $6 \mathrm{~h}(\mathrm{a}, \mathrm{b}, \mathrm{c}), 24 \mathrm{~h}(\mathrm{~d}, \mathrm{e}, \mathrm{f})$, and 9 days $(\mathrm{g}, \mathrm{h}, \mathrm{i})$. Acetylated $\boldsymbol{\alpha}$-tubulin $(\mathrm{a}, \mathrm{c}, \mathrm{e})$; NDPK $(\mathrm{b}, \mathrm{d}, \mathrm{f})$. Arrows in $b$ and e show some of the primary cilia staining for NDPK; arrows in $h$ point to exceptionally long primary cilia. Arrowhead in b indicates a short cilium that stains strongly with anti acetylated $\alpha$-tubulin but lacks NDPK. A mitotic spindle stained by anti acetylated $\alpha$-tubulin is indicated by an asterisk ( $\left.{ }^{*}\right)$ in $\mathrm{d}$. 
NDPKs A and B, as well as the amphibian NDPKs, are by and large cytosolic proteins. Nevertheless, they are also found in the nucleus, in the plasma membrane of stimulated cells, and in association with the centrosome, cytoskeletal elements, vesicular compartments and sperm flagella (see Introduction). Biochemical approaches based on isolated 9+2 cilia and flagella have been useful in the identification of ciliary components, their localization inside the cilium and the interactions governing their import into these organelles. However, when we started this work, there were no published methods for isolation of primary cilia, most probably because in the majority of cell lines there is only one per cell, they are diminutive, and accordingly comprise a minute fraction of total cell volume (Gallagher, 1980). The feasibility of a successful isolation is higher in A6 cells, which have a high incidence of long primary cilia when cultured for an extended period post-confluence (Figs. 21 and 24). Based on the observation that shear forces can easily lead to deciliation of specimens (Gallagher, 1980), we developed a procedure for isolating primary cilia from A6 cells using brief mechanical agitation in saline followed by differential centrifugation. To prevent damage to the cilia, we avoided detergents and chemicals such as dibucaine, as well as extremes of $\mathrm{pH}$ and high calcium, which are common in cilia/flagella isolation (Watson and Hopkins, 1962; Rosenbaum and Child, 1967; Linck, 1973; Anderson, 1974; Hastie, 1986; Zhang et al., 1991). This mild approach also prevented lysis of cells, which remain largely intact after the procedure (Fig. 26), thus reducing contamination of the primary cilium fraction with proteins from the cell body. This method was also successfully used to remove primary cilia from MDCK. Because the latter require weeks of time in culture 


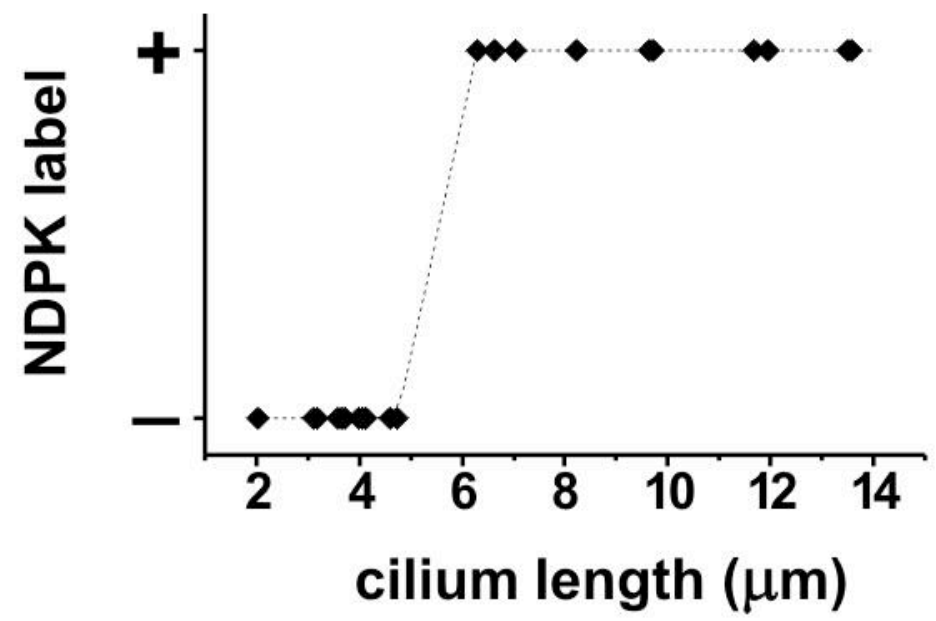

Figure 25. Translocation of NDPK into the primary cilium is length-dependent. The apparent lengths of cilia were measured in images of cells treated as in Figure 24, and fixed combed after intervals of 6 to $8 \mathrm{~h}$ post-plating. Each cilium was measured using the image stained for acetylated $\alpha$-tubulin and then categorized by visual inspection of 3-5 thru-focus images as positive or negative for NDP kinase; the result (NDPK - or +) was plotted against length.

\section{Before}

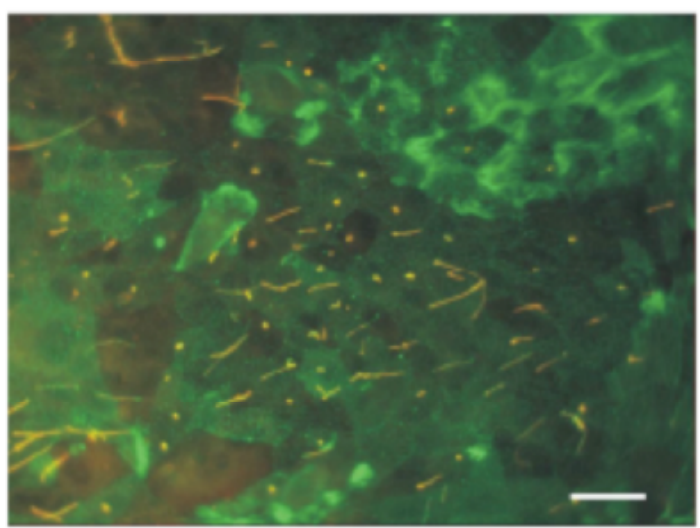

After

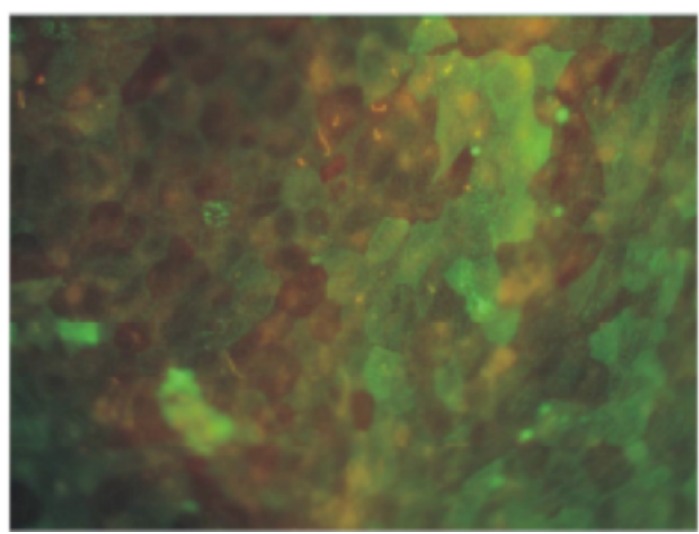

Figure 26. Cells remain intact after removal of primary cilia. A6 cells before and after deciliation were fixed and stained with anti-NDPK (green) and anti-acetylated $\alpha$ tubulin (red). Bar $=25 \mu \mathrm{M}$ 
to grow cilia of lengths suitable for biochemical and developmental studies, most of the results reported here were obtained using A6 cells.

Analysis of the protein composition of the preparation obtained through this method by SDS-PAGE and staining with Coomassie Blue demonstrates that this fraction differs considerably from whole cell extracts. The pattern seen in Figure $27 \mathrm{a}$ is very reproducible between different preparations, with the most prominent bands in the whole cilia fraction migrating at $31,47,54,60,82,105$ and $146 \mathrm{kDa}$.

Immunoblot analysis of the primary cilium fraction is shown in Figure $27 \mathrm{~b}$. Comparison of the signal for the ciliary marker acetylated $\alpha$-tubulin in equal amounts (10 $\mu \mathrm{g}$ ) of whole cell extracts and primary cilium preparations (Fig. 27b, upper panel) shows a strong band at $54 \mathrm{kDa}$ in the isolated primary cilium, but no detectable reaction with the whole cell extract, as expected from an antigen present at low levels in cells (Piperno et al., 1987). Thus, the procedure used here yields a fraction highly enriched in a specific ciliary marker. More importantly, isolated primary cilia contain substantial amounts of NDPK, although proportionately less than whole cell lysates (70\%; Fig. 27b, lower panel). Note that although three NDPKs have been identified in Xenopus (Ouatas et al, 1997), these proteins are virtually identical in terms of amino acid sequence ( $>96 \%$ identity), size and isoelectric point and are not resolved by SDS-PAGE, thus only one band of about $18 \mathrm{kDa}$ is seen in immunoblots.

Assay of the primary cilium fraction for NDPK activity shows a specific activity of $1 \mu \mathrm{mol} / \mathrm{mg} / \mathrm{min}$, about $60 \%$ of the activity measured in whole cell lysates, 1.7 

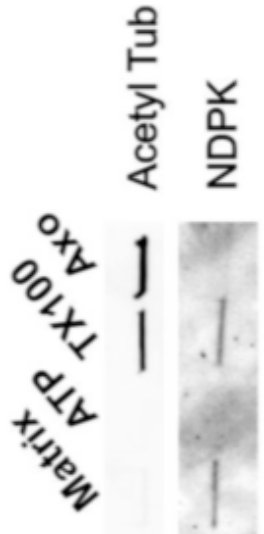

0 $\frac{x}{0}$

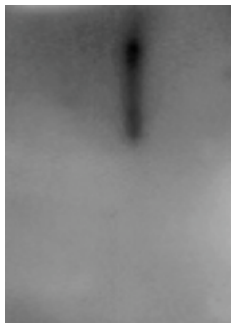

옴

응

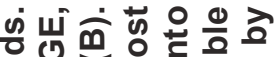

중

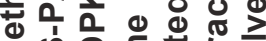

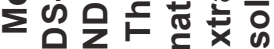

क为

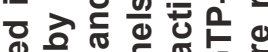

으의

$\geq$

\&

車

웡

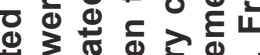

\%

응 웡

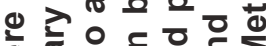

일

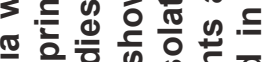

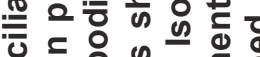
>등

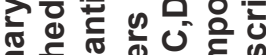
द.

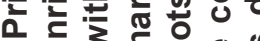

0 3 응 용 흔 은 >

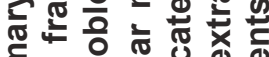
을 흐눌

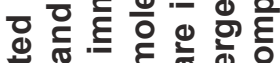

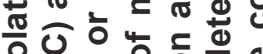

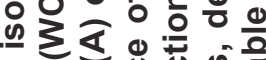
○ 0 造 ธ。

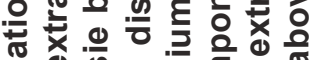

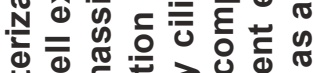

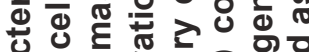

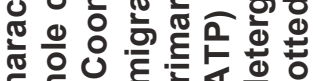
든 区丁

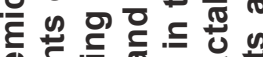
d

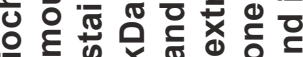
两

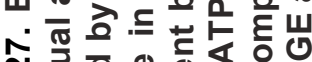
N 닌

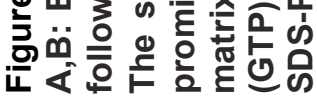


$\mu \mathrm{mol} / \mathrm{mg} / \mathrm{min}$, and in agreement with the estimate made by densitometry of immunoblots. Therefore, ciliary NDPK is active as an enzyme.

\subsubsection{NDPK is present in the matrix and membranes of primary cilia isolated from}

\section{A6 cells.}

Our next objective was to determine whether NDPK is present in primary cilia as a soluble matrix protein, an axonemal component or in association with the surface membrane. To do so, we fractionated isolated primary cilia by a procedure involving sequential freeze/thaw, nucleotide treatment and detergent extraction. Immunoblotting of these fractions indicates that NDPK is present in the matrix and membranes, but not in the axoneme-containing pellet (Fig. 27c). The subciliary distribution of NDPKs in primary cilia differs markedly from to the axonemal association of NM23-5, 7 and 9 in 9+2 cilia and flagella (Ostrowski et al.; 2002, Munier et al., 2003). Further, this lack of association with the axoneme confirms the observations made in intact cells, where the staining pattern of NDPK differs from that of acetylated $\alpha$-tubulin (Fig. 23). Indeed, the bulk of acetylated $\alpha$-tubulin was found in the axoneme, with lesser amounts in the detergent extract and only traces in the matrix (Fig. 27c). The presence of acetylated $\alpha-$ tubulin in fractions other than the axoneme was at first unexpected, given that acetylation of $\alpha$-tubulin takes place after microtubules polymerize (Piperno et al., 1987). However, Qin et al. (2004) have also found this protein in the membrane+matrix fraction of Chlamydomonas flagella, and suggested that soluble acetylated $\alpha$-tubulin is a by-product of axonemal turnover, being returned to the cell body by retrograde intraflagellar transport (IFT). 
Because nucleotides release NDPK from intracellular membrane vesicles (Gallagher et al., 2003; Mitchell et al., submitted), we sought to determine whether this is also the case for primary cilia membranes. However, there was no release of NDPK when we extracted the membrane+axoneme fraction with $1 \mathrm{mM} \mathrm{GTP}$ or $10 \mathrm{mM}$ ATP (Fig. 27 $\mathrm{c}, \mathrm{d})$, indicating that distinct mechanisms underlie its binding to ciliary and intracellular membranes.

\subsubsection{Appearance of isolated primary cilia}

Preparations of primary cilia were examined by microscopy to assess yield and composition. Figure 28a shows that the fraction contains a large number of structures of varying length with the expected tubular shape. Most of these structures have a swelling at one end, which is probably the bulbous tip frequently seen in renal primary cilia (Fig. 28a). In some cases, the tubular portion attached to the rounded tip is very short; furthermore, numerous structures have the characteristics of these globular tips, but no visible axoneme. Immunofluorescence analysis of the isolated primary cilium fraction confirms that the preparation contains not only typical cilia, but also round structures of roughly the size of ciliary tips (Fig. 28b) that stain for both acetylated $\alpha$-tubulin and NDPK (Fig. 28c). Structures that contain only NDPK, presumably cell debris, are also visible (Fig. 28c), and constitute an estimated $40-60 \%$ of the isolated primary cilia fraction.

Taken together, the data suggest that our isolation protocol causes rupture of the ciliary stem at different positions, and that often this break takes place at the very base of the distal swelling. The resulting ciliary tip is of particular interest, because it is the site 

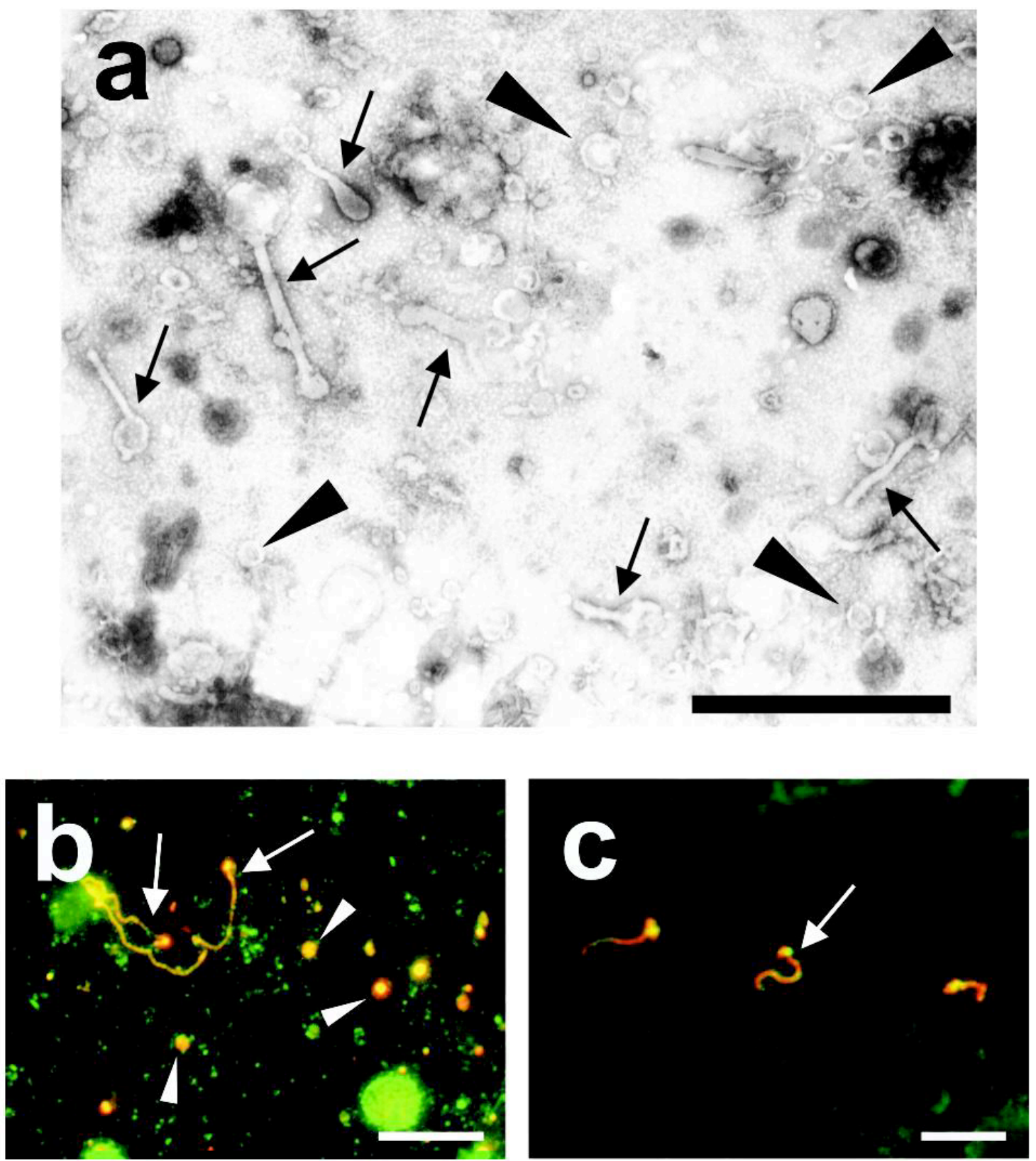

Figure 28. The primary cilia fraction contains whole cilia and ciliary tips. Primary cilia were isolated as in Methods. a: Negatively stained samples were examined by electron microscopy. b: Isolated primary cilia preparations were fixed and doubly stained with antibodies to acetylated $\alpha$-tubulin and NDPK. c: A6 cells were fixed and stained as in b. Red: acetylated $\alpha$-tubulin; green, NDP kinase; yellow, overlap regions. Arrows indicate primary cilia, and arrowheads point to ciliary tip-like structures. Bars: (a) $5 \mu \mathrm{m}$; (b,c) $25 \mu \mathrm{m}$. 
of microtubule assembly and disassembly, as well as the area where IFT particles release their cargo, reload, and reverse direction, returning to the base of the cilium (Rosenbaum and Witman, 2002).

\subsubsection{Expression of EGFP-tagged NDPK}

In attempt to observe NDPK's entry into primary cilia, we transfected NIH-3T3 and A6 cells with EGFP-tagged NDPK's A and B. The vector utilized here was successfully employed by Milon et al. (2000) to confirm the mitochondrial localization of NDPK D in mammalian cells. The caveat is that the molecular weight of monomeric NDPK A and B is about $20 \mathrm{kDa}$, much lower than that of EGFP (29 kDa), and the size of the tag could conceivably affect the localization patterns of the protein under study. Moreover, because NDPK is a hexamer, hypothetically up to six EGFP tags (Fig. 29) could end up on one NDPK unit, greatly increasing its volume, and potentially changing both its mobility within the cell and its ability to interact with other proteins. Indeed, this seems to be the case, as in 5 separate experiments, EGFP-tagged NDPK A and B failed to enter primary cilia in NIH-3T3 (Fig. 30) and A6 (Fig. 31) cells, although both proteins retained their normal localization pattern in the cell periphery and cytosol (Figs. 30 and 31). Thus, the addition of the EGFP moiety affects adversely the portion of NDPK that carries the signal needed for entrance into the primary cilium. This confirms that the primary cilium is very selective in the components that it admits.

\subsubsection{Binding partners of NDPK and another NM23/NDPK are present in primary cilia}




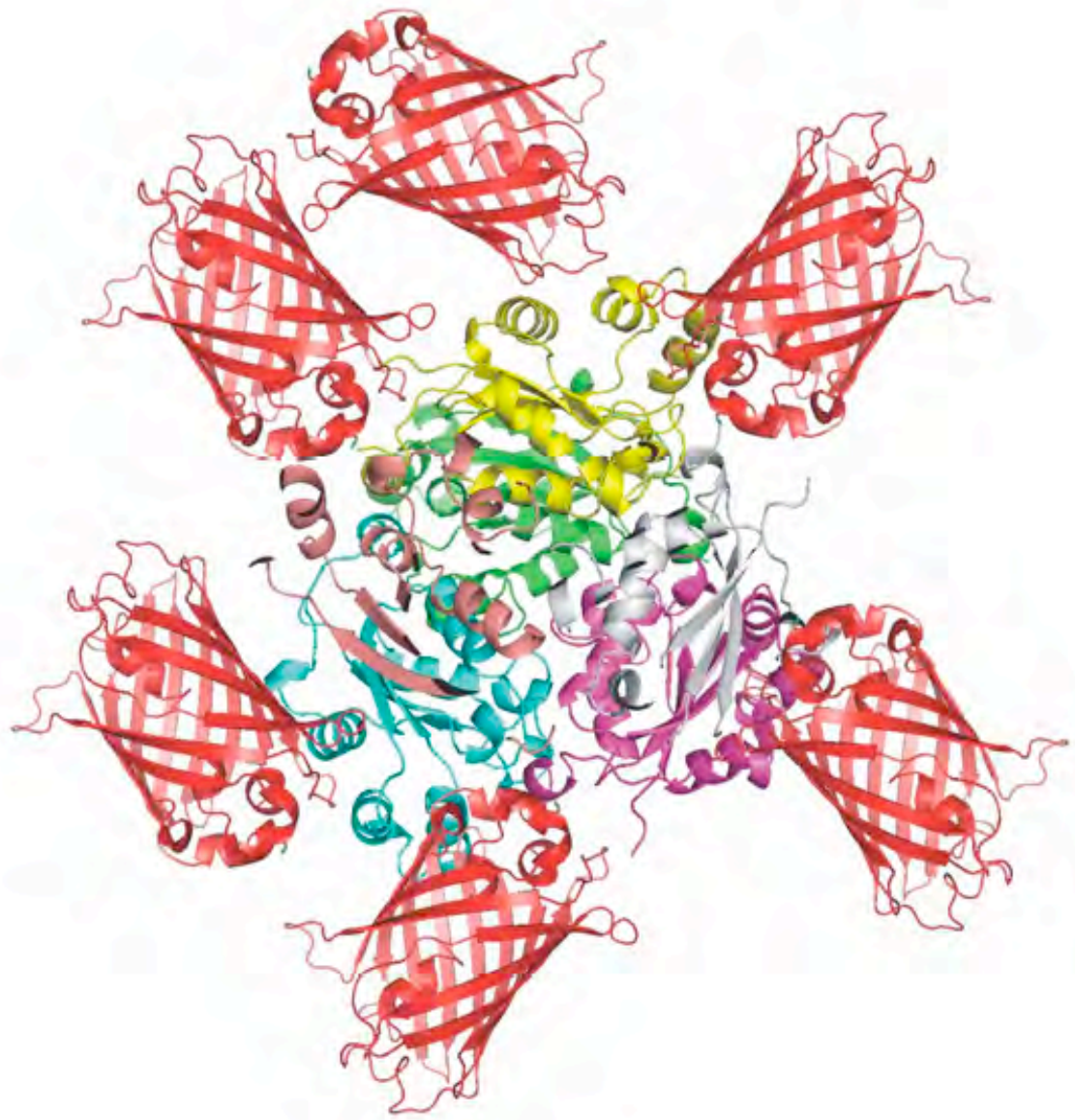

Figure 29. Model of EGFP-tagged NDPK with an EGFP tag on each monomer. 

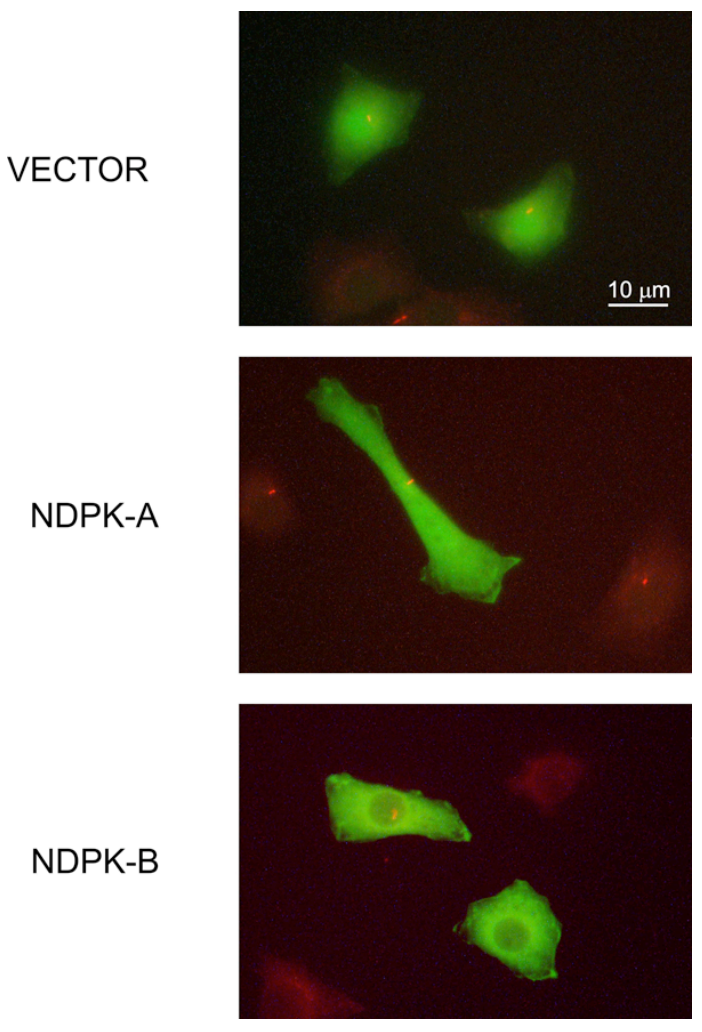

Figure 30. EGFP-tagged NDPK does not enter the primary cilium of NIH-3T3 cells. NIH-3T3 cells transfected with EGFP-tagged hNDPKs A and B and stained with anti $\boldsymbol{\alpha}$-acetylated tubulin (red).
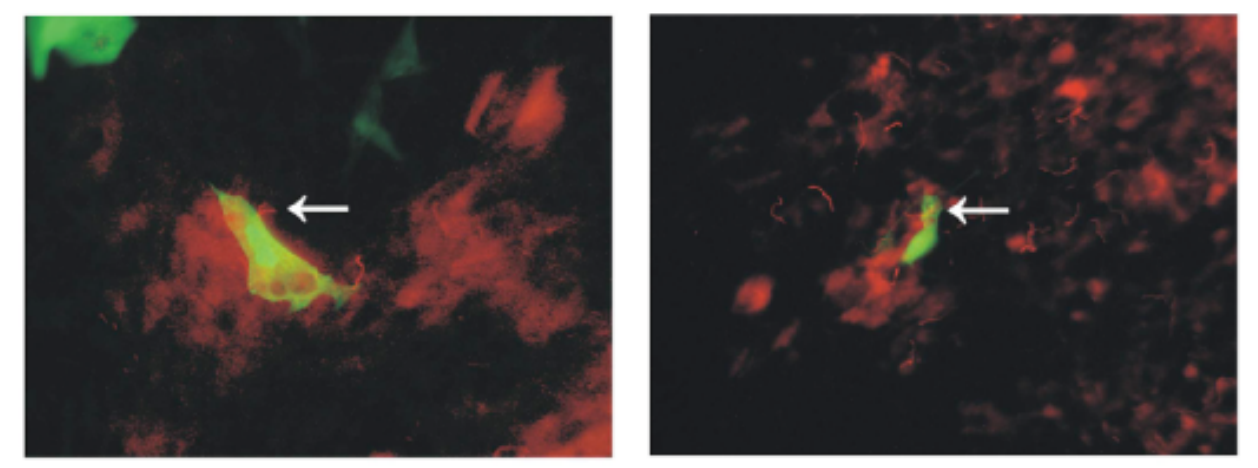

Figure 31. EGFP-tagged NDPK does not enter the primary cilium of A6 cells. A6 cells transfected with EGFP-tagged hNDPK $A$ and stained with anti $\alpha$-acetylated tubulin (red). 
As the NDPK in primary cilia membranes is not released by nucleotides in the same manner as seen with NDPK in Mt/Ves, it may be targeted to the primary cilium by association with other proteins. We used immunofluorescence methods to test for NDPK binding partners, including the mitotic kinase Aurora A (Du and Hannon, 2002), the small GTPase Arf6, which recruits it to cell-cell junctions (Palacios et al., 2002), the Rac guanine nucleotide exchange factor Tiam1 (Otsuki et al., 2001) and dynamin (Krishnan et al., 2001; Baillat et al., 2002). We found no evidence of primary cilium localization of any of these proteins in A6 or MDCK cells. However, we did find that four NDPK binding partners previously identified by our lab [creatine kinase (CK), glyceraldehyde3-phosphate dehydrogenase (GAPD), pyruvate kinase (PK) and vimentin (VIM); Otero, 1997] localize to primary cilia. Figure 32 shows images of MDCK and A6 cells stained for PK, VIM, CK and GAPD, and reveals that all four proteins are present in primary cilia of renal cells. The intermediate filament protein vimentin interacts with the cytoplasmic portion of polycystin-1 in kidney epithelial cells (Xu et al., 2001) and may participate in the signaling network associated with the primary cilium. The metabolic enzymes are probably involved in ATP production for molecular motors, and like NDPK in primary cilia, pyruvate kinase is present in the membrane and matrix fractions of flagella (Mitchell et al., 2005). Thus, any of these NDPK binding partners may mediate its association with specific intra-ciliary compartments.

Because NM23-H5 is known to localize to sperm flagella, we also tested for its presence in primary cilia. Figure 33 shows that NM23-H5 localizes only to a few cilia in which it stains the middle, but not the top or bottom of the cilia, so its staining pattern 


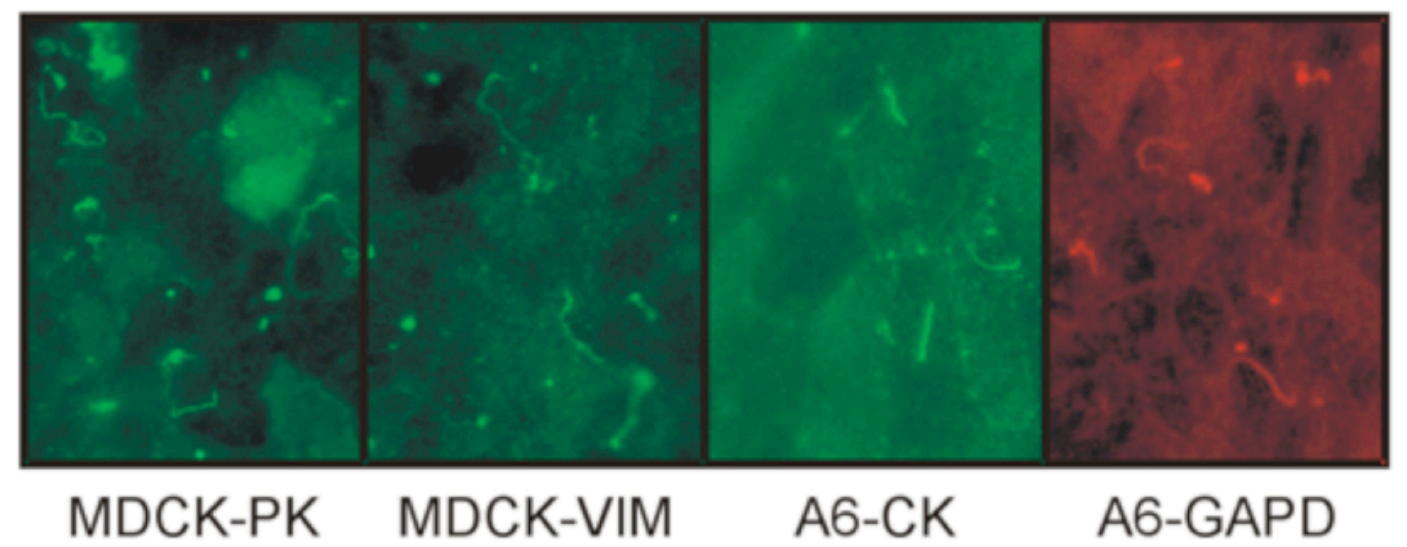

Figure 32. Binding partners of NDPK localize to primary cilia. MDCK and A6 cells stained for pyruvate kinase (PK), vimentin (VIM), creatine kinase (CK), and glyceraldehyde-3-phosphate dehydrogenase (GAPD).

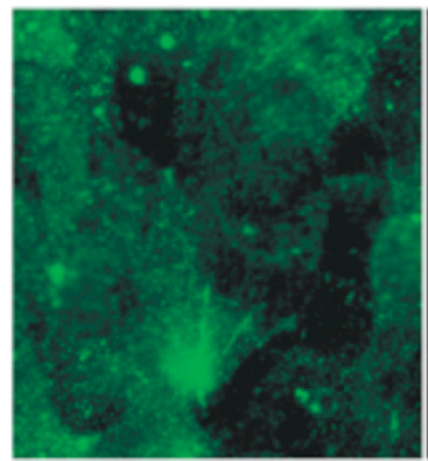

H5

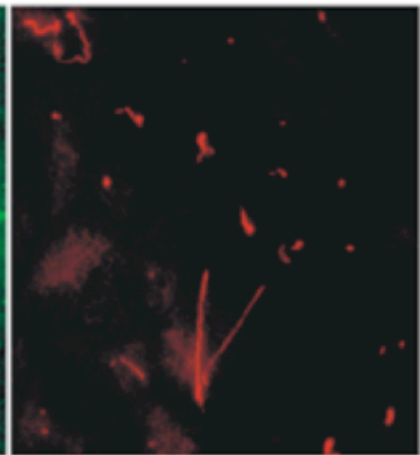

AcT

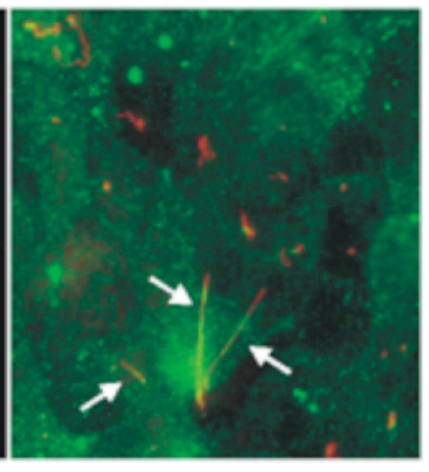

merged

Figure 33. NM23-H5 localizes to some, but not all, primary cilia. A6 cells stained for H5 (green) and acetylated $\alpha$-tubulin (red). 
overlaps only partially with that of acetylated $\alpha$-tubulin. Therefore, NM23-H5 probably does not affect the localization of cytosolic NDPKs in primary cilia. Also, the function of NM23-H5 in primary cilia is likely to be distinct from that of NDPKs A and B.

\subsection{Discussion}

Our data indicate that conventional NDPKs are present in primary cilia of mammalian and amphibian cells, and are found in cilia from different cell types, namely fibroblasts and epithelial cells. These results were confirmed by the finding of NDPK in the matrix and membrane fractions of preparations enriched in primary cilia. Therefore, NDPKs are universal components of primary cilia. Taken together with the finding that NDPKs A and B are also observed in sperm flagella (Munier et al., 2003), we expect that these proteins will be eventually found in all cilia and flagella.

Cilia and flagella are made of 200-250 proteins (Rosenbaum and Witman, 2002), which are synthesized in the cell body. The compositions of the ciliary membrane and matrix are distinct from those of the plasma membrane and cytosol, indicating that strict sorting and targeting mechanisms allow only a limited set of proteins into these structures. This selectivity appears to be accomplished through a "filter" at the base of cilia, as well as through specific interactions with IFT particles (Rosenbaum and Witman, 2002; Scholey, 2003). IFT is the process by which motors such as kinesin and dynein move large multimeric complexes of proteins along the axoneme using microtubule tracks. The possibility of a link between NDPK and IFT is suggested by the punctate staining pattern of NDPK along the ciliary shaft, which is reminiscent of that seen with IFT proteins (Cole et al., 1998; Deane et al., 2001). Furthermore, the intraflagellar 
distribution of NDPK to the matrix and membrane fractions of primary cilia, but not to the axoneme, is also typical of components of IFT particles and motors (Cole et al., 1998; Qin et al., 2004). On the other hand, IFT proteins are characteristically concentrated at the base of primary cilia (Cole et al. 1998; Deane et al., 2001; Taulman et al., 2001), and NDPK is not. Hence, we expect that NDPK is a cargo of IFT, but not an IFT component. Axonemal length also seems to influence the function of the sensory machinery present in cilia. For example, measurements of the mechanosensory ability of MDCK cells during reciliation show that recovery of the flow-induced $\mathrm{Ca}^{2+}$ response takes place only when the growing cilia reach a critical length of 3-4 $\mu \mathrm{m}$ (Praetorius and Spring, 2003), which is comparable to the 5-6 $\mu \mathrm{m}$ threshold we find for NDPK translocation into the cilia of A6 cells. Furthermore, Nauli et al. (2003) found that the flow-sensing response of embryonic kidney cells, mediated by polycystins 1 and 2, is absent from cells with few, underdeveloped short cilia. By analogy, the length-dependent translocation of NDPK into the primary cilium of A6 cells suggests that it plays a role in the sensory machinery of cilia.

The observation that axonemal microtubules are dynamic structures and are constantly assembling and disassembling (Marshall and Rosenbaum, 2001; Song and Dentler, 2001) suggests a possible role for NDPK in cilia elongation and maintenance. Particularly, microtubule turnover leads to the hydrolysis of considerable amounts of GTP, but cellular GTP levels are 5-10-fold lower than those of ATP, and diffusion may not be sufficient to provide an adequate $[\mathrm{GTP}] /[\mathrm{GDP}]$ ratio along a thin, long cilium, in particular at the tip. Conventional NDPKs can regenerate GTP from GDP and ATP at 
high rates, and are a source of GTP for endocytosis (Krishnan et al., 2001; Palacios et al., 2002). We suggest that translocation of NDPK into growing cilia reflects increased GTP demand during microtubule disassembly, which would become significant only after the cilium reaches a preset length. Transport of NDPK into cilia at this stage would provide a source of GTP and allow for accelerated turnover of axonemal tubulin. Additionally, GTP regeneration by NDPK might sustain the activity of ciliary GTPases involved in sensory signaling through G-protein-coupled receptors (Pazour and Witman, 2003) and in IFT (e.g., IFT27, Rosenbaum and Witman, 2002).

In summary, our immunofluorescence localization and biochemical studies provide evidence showing that cytosolic NDPKs are standard components of primary cilia in fibroblasts and epithelial cells, and that entry of NDPKs into the cilium takes place only after a critical length is reached. Furthermore, the development of the first procedure for the isolation of a fraction enriched in primary cilia from A6 cells allowed us to determine that NDPKs are associated with the membranous and soluble compartments of cilia where transport and signaling processes take place, a location consistent with a role in GTP replenishment. 


\section{CHAPTER 5: CONCLUSION AND REFERENCES}

\subsection{Conclusion}

NDPK is involved in numerous essential cellular processes. It is therefore likely that it regulates and is regulated by multiple proteins and other components such as lipids. Determining its spatial distribution inside cells is essential to the determination of how it performs its various tasks. Our studies of the mechanisms by which NDPK associates with different organelles may be relevant to the understanding of its role in normal cellular processes and their alterations in human disease.

Our finding that NDPK is translocated when the Rac1 pathway is activated by receptor tyrosine kinases and G protein-coupled receptors shows that NDPK's cellular localization is a dynamic process, influenced by external stimuli. More importantly, what initially appeared to be a static, constitutive association with intracellular vesicles was revealed to be another dynamic process, relying on a novel, direct interaction of NDPK with lipids and controlled by NDPK's catalytic cycle and the energy charge of the cell.

Finally, the discovery that NDPK is a component of the primary cilium that enters this organelle at a precise stage of ciliary development implies again that the intracellular localization of NDPK is dynamic and responsive to specific signals. As relatively few proteins are found in primary cilia, and NDPK is one of them, it must serve an important function in the working of this sensory organelle.

Overall, the results presented here add to what is an increasingly complex view of the function of NDPKs, once more confirming that these plain, cytosolic housekeeping 
enzymes are essential components of a variety of events, able to change their intracellular location in response to both external and internal cues. 


\subsection{References}

Alieva IB, Gorgidze LA, Komarova YA, Chernobelskaya OA and Vorobjev IA. (1999). Experimental model for studying the primary cilia in tissue culture cells. Membr Cell Biol 12, 895-905.

Anderson RG. (1974). Isolation of ciliated or unciliated basal bodies from the rabbit oviduct. J Cell Biol 60, 393-404.

Antignani A and Youle RJ. (2008). Endosome fusion induced by diphtheria toxin translocation domain. Proc Natl Acad Sci USA 105, 8020-8025.

Arnaud-Dabernat S, Masse K, Smani M, Peuchant E, Landry M, Bourbon PM, Le Floch R, Daniel JY and Larou M. (2004). Nm23-M2/NDP kinase B induces endogenous c-myc and nm23-M1/NDP kinase A overexpression in BAF3 cells. Both NDP kinases protect the cells from oxidative stress-induced death. Exp Cell Res 301, 293-304.

Arnaud-Dabernat S, Bourbon PM, Dierich A, Le Meur M and Daniel JY. (2003). Knockout mice as model systems for studying nm23/NDPK gene functions. Application to the $n m 23-M 1$ gene. J Bioenerg Biomembr 35, 19-30.

Baillat G, Gaillard S, Castets F and Monneron A. (2002). Interactions of phocein with nucleoside-diphosphate kinase, Eps15 and dynamin I. J Biol Chem 277, 1896118966.

Barraud P, Amrein L, Dobremez E, Dabernat S, Masse K, Larou M, Daniel JY and Landry M. (2002). Differential expression of $n m 23$ genes in adult mouse dorsal root ganglia. J Comp Neurol 444, 306-323. 
Besant PG and Attwood PV. (2005). Mammalian histidine kinases. Biochem Biophys Acta 1754, 281-290.

Boissan M, Wendum D, Arnaud-Dabernat S, Munier A, Debrav M, Lascu I, Daniel J-Y, and Lacombe M-L. (2005). Increased lung metastasis in transgenic NM23null/SV40 mice with hepatocellular carcinoma. Cancer Inst 97, 836-845.

Bominaar AA, Tepper AD and Veron M. (1994). Autophosphorylation of nucleoside diphosphate kinase on non-histidine residues. FEBS Lett 353, 5-8.

Brailov I, Bancila M, Brisorgueil MJ, Miquel MC, Hamon M and Verge D. (2000). Localization of 5-HT(6) receptors at the plasma membrane of neuronal cilia in the rat brain. Brain Res 872, 271-275.

Bremser M, Nickel W, Schweikert M, Ravazzola M, Amherdt M, Hughes CA, Söllner TH, Rothman JE and Wieland FT. (1999). Coupling of coat assembly and vesicle budding to packaging of putative cargo receptors. Cell 96, 495-506.

Cervoni L, Egistelli L, Eufemi M, d'Abusco AS, Altieri F, Lascu I, Turano C and Giartosia A. (2006). DNA sequences acting as binding sites for NM23/NDPK proteins in melanoma M14 cells. $J$ Cell Biochem 98, 421-428.

Cervoni L, Pietrangeli P, Chichiarelli S, Altieri F, Egisteli L, Turano C, Lascu I and Giartosio A. (2003). In vivo cross-linking of nm23/nucleoside diphosphate kinase to the PDGF-A promoter. Mol Biol Rep 30, 33-40.

Cervoni L, Lascu I, Xu Y, Gonin P, Morr M, Merouani M, Janin J and Giartosio A. (2001). Binding of nucleotides to nucleoside diphosphate kinase: a calorimetric study. Biochemistry 40, 4583-4589. 
Cho SJ, Lee NS, Jung YS, Lee H, Lee KJ, Kim E and Chae SK. (2001). Identification of structural domains affecting transactivation potential of Nm23. Biochem Biophys Res Comm 289, 738-743.

Chowdhury D, Beresford PZ, Zhang D, Sung J-S, Demple B, Perrino FW and Lieberman J. (2006). The exonuclease TREX1 is in the SET complex and acts in concert with NM23-H1 to degrade DNA during granzyme A-mediated cell death. Mol Cell 23, $133-142$.

Christensen ST, Pedersen LB, Schneider L and Satir P. (2007). Sensory cilia and integration of signal transduction in human health and disease. Traffic 8, 97-109.

Cole DG, Diener DR, Himelblau AL, Beech PL, Fuster JC and Rosenbaum JL. (1998). Chlamydomonas kinesin-II-dependent intraflagellar transport (IFT): IFTparticles contain proteins required for ciliary assembly in Caenorhabditis elegans sensory neurons. J Cell Biol 141, 993-1008.

Corbit KC, Aanstad P, Singla V, Norman AR, Stainier DYR and Reiter JF. (2005). Vertebrate smoothened functions at the primary cilium. Nature 437, 1018-1021.

Dammai V, Adryan B, Lavenburg KR and Hsu T. (2003). Drosophila awd, the homolog of human nm23, regulates FGF receptor levels and functions synergistically with shi/dynamin during tracheal development. Genes and Dev 17, 2812-2824.

Davenport JR, Watts AJ, Roper VC, Croyle MJ, van Groen T, Wyss JM, Nagy TR, Kesterson RA and Yoder BK. (2007). Disruption of intraflagellar transport in adult mice leads to obesity and slow-onset cystic kidney disease. Curr Biol 17, 1586-1594. 
Davenport JR and Yoder BK. (2005). An incredible decade for the primary cilium: a look at a once-forgotten organelle. Am J Physiol Renal Physiol 289, F1159-1169.

Deane JA, Cole DG, Seeley ES, Diener DR and Rosenbaum JL. (2001). Localization of intraflagellar transport protein IFT52 identifies basal body transitional fibers as the docking site for IFT particles. Curr Biol 11, 1586-1590.

Dearolf CR, Hersperger E and Shearn A. (1988a). Developmental consequences of awdb3, a cell-autonomous lethal mutation of Drosophila induced by hybrid dysgenesis. Dev Biol 129, 159-168.

Dearolf CR, Tripoulas N, Biggs J and Shearn A. (1988b). Molecular consequences of awdb3, a cell-autonomous lethal mutation of Drosophila induced by hybrid dysgenesis. Dev Biol 129, 169-178.

Degani S, Balzac F, Brancaccio M, Guazzone S, Retta SF, Silengo L, Eva A and Tarone G. (2002). The integrin cytoplasmic domain-associated protein ICAP-1 binds and regulates Rho family GTPases during cell spreading. J Cell Biol 156, 377-387.

Du J and Hannon GJ. (2002). The centrosomal kinase Aurora-A/STK15 interacts with a putative tumor suppressor NM23-H1. Nucleic Acids Res 30, 5465-5475.

Duriez B, Duquesnoy P, Escudier E, Bridoux AM, Escalier D, Rayet I, Marcos E, Vojtek AM, Bercher JF and Amselem S. (2007). A common variant in combination with a nonsense mutation in a member of the thioredoxin family causes primary ciliary dyskinesia. Proc Natl Acad Sci USA 104, 3336-3341.

Engel M, Veron M, Theisinger B, Lacombe ML, Seib T, Dooley S and Welter C. (1995). A novel serine/threonine-specific protein phosphotransferase activity of 
Nm23/nucleoside-diphosphate kinase. Eur J Biochem 234, 200-207.

Epand RF, Schlattner U, Wallimann T, Lacombe ML and Epand RM. (2007). Novel lipid transfer property of two mitochondrial proteins that bridge the inner and outer membranes. Biophys J 92, 126-137.

Fan Z, Beresford PJ, Oh DY, Zhang D and Lieberman J. (2003). Tumor suppressor NM23-H1 is a granzyme A-activated DNase during CTL-mediated apoptosis, and the nucleosome assembly protein SET is its inhibitor. Cell 112, 659-672.

Fournier HN, Albiges-Rizo C and Block MR. (2003). New insights into nm23 control of cell adhesion and migration. J Bioenerg Biomembr 35, 81-87.

Fournier HN, Dupe-Manet S, Bouvard D, Lacombe ML, Marie C, Block MR and Albiges-Rizo C. (2002). ICAP-1alpha interacts directly with the metastasis suppressor nm23-H2 and both proteins are targeted to newly formed cell adhesion sites upon integrin engagement. J Biol Chem 277, 20895-20902.

Freije JMP, Blay P, MacDonald NJ, Manrow RE and Steeg PS. (1997). Sitedirected mutagenesis of NM23-H1. Mutations lacking motility suppressive capacity upon transfection are deficient in histidine dependent protein phosphotransferase pathways in vitro. $J$ Biol Chem 272, 5525-5532.

Fullerton AT, Bau MY, Conrad PA and Bloom GS. (1998). In vitro reconstitution of microtubule plus end-directed, GTPgammaS-sensitive motility of Golgi membranes. Mol Biol Cell 9, 2699-2714.

Gallagher BC, Parrott KA, Szabo G and Otero AS. (2003). Receptor activation regulates cortical, but not vesicular localization of NDP kinase. J Cell Sci 116, 3239-3250. 
Gallagher BC. 1980. Primary cilia of the corneal endothelium. Am J Anat 159, 475-484.

Ganley IG, Carroll K, Bittova L and Pfeffer S. (2004). Rab9 GTPase regulates late endosome size and requires effector interaction for its stability. Mol Biol Cell 15, 5420-5430.

Garces E and Cleland WW. (1969). Kinetic studies of yeast nucleoside diphosphate kinase. Biochemistry 7, 633-640.

Garin J, Diez R, Kieffer S, Dermine JF, Duclos S, Gagnon E, Sadoul R, Rondeau C and Desjardins M. (2001). The phagosome proteome: insight into phagosome functions. J Cell Biol 152, 165-180.

Gilles AM, Presecan E, Vonica A and Lascu I. (1991). Nucleoside diphosphate kinase from human erythrocytes. Structural characterization of the two polypeptide chains responsible for heterogeneity of the hexameric enzyme. J Biol Chem 266, 8784-8789.

Goltz JS, Wolkoff AW, Novikoff PM, Stockert RJ and Satir P. (1992). A role for microtubules in sorting endocytic vesicles in rat hepatocytes. Proc Natl Acad Sci USA 89, 7026-7030.

Hartsough MT, Morrison DK, Salerno M, Palmieri D, Ouatas T, Mair M, Patrick J and Steeg PS. (2002). Nm23-H1 metastasis suppressor phosphorylation of kinase suppressor of Ras via a histidine protein kinase pathway. J Biol Chem 277, 32389-32399.

Hartsough MT and Steeg PS. (2000). Nm23/nucleoside diphosphate kinase in human cancers. J Bioenerg Biomembr 32, 301-308. 
Hastie AT, Dicker DT, Hingley ST, Kueppers F, Higgins ML and Weinbaum G. (1986). Isolation of cilia from porcine tracheal epithelium and extraction of dynein arms. Cell Motil Cyto 6, 25-34.

Hippe H-J and Wieland T. (2006). High energy phosphate transfer by NDPK B/G-betagamma complexes - an alternative signaling pathway involved in the regulation of basal cAMP production J Bioenerg Biomembr 38, 197-203.

Hou X, Mrug M, Yoder B, Lefkowitz E, Kremmidiotis G, D’Eustachio P, Beier D and Guay-Woodford L. (2002). Cystin, a novel cilia-associated protein, is disrupted in the cpk mouse model of polycystic kidney disease. J Clin Invest 109, 533-540.

Hsu T, Adereth Y, Kosse N and Dammai V. (2006). Endocytic function of von-HippelLindau tumor suppressor protein regulates surface localization of fibroblast growth factor receptor 1 and cell motility. J Biol Chem 281,12069-12080.

Huitorel P, Simon C and Pantaloni D. (1984). Nucleoside diphosphate kinase from brain. Purification and effect on microtubule assembly in vitro. Eur J Biochem 144, 233241.

Islam K and Burns RG. (1985). Microtubules and nucleoside diphosphate kinase. Nucleoside diphosphate kinase binds to co-purifying contaminants rather than to microtubule proteins. Biochem J 232, 651-656.

Jacobs M and Huitorel P. (1979). Tubulin-associated nucleoside diphosphokinase. Eur J Biochem. 99, 613-622.

Janin J, Dumas C, Morera S, Xu Y, Meyer P, Chiadmi M and Cherfils J. (2000). Threedimensional structure of nucleoside diphosphate kinase. J Bioenerg Biomembr 32, 
215-226.

Jung H, Seona H-A and Hal H. (2007). NM23-H1 tumor suppressor and its interacting strap activate p53 function. J Biol Chem 282, 35293-35307.

Kaetzel DM, Zhang Q, Yang M, McCorkle JR, Ma D and Craven RJ. (2006). Potential roles of 3-5' exonuclease activity of NM23-H1 in DNA repair and malignant progression. J Bioenerg Biomembr 38, 163-167.

Kapetanovich L, Baughman C and Lee TH. (2005). Nm23 facilitates coat protein complex II assembly and endoplasmic reticulum export in mammalian cells. Mol Biol Cell 16, 835-848.

Kikkawa S, Takahashi K, Takahashi K, Shimada N, Ui M, Kimura N and Katada T. (1992). Activation of nucleoside diphosphate kinase by mastoparan, a peptide isolated from wasp venom. FEBS Lett 305, 237-240.

Kim YI, Park S, Jeoung DI and Lee H. (2003). Point mutations affecting the oligomeric structure of Nm23-H1 abrogates its inhibitory activity on colonization and invasion of prostate cancer cells. Biochem Biophys Res Comm 307, 281-289.

Kimura N, Shimada N, Fukuda M, Ishijima Y, Miyazaki H, Ishii A, Takagi Y and Ishikawa N. (2000). Regulation of cellular functions by nucleoside diphosphate kinases in mammals. J Bioenerg Biomembr 32, 309-315.

Krishnan KS, Rikhy R, Rao S, Shivalkar M, Mosko M, Narayanan R, Etter P, Estes PS and Ramaswami M. (2001). Nucleoside diphosphate kinase, a source of GTP, is required for dynamin-dependent synaptic vesicle recycling. Neuron 30, 197-210. 
Lacombe ML, Milon L, Munier A, Mehus JG, Lambeth DO. (2000). The human Nm23/nucleoside diphosphate kinases. J Bioenerg Biomembr 32, 247-258.

Lacombe ML, Wallet V, Troll H and Veron M. (1990). Functional cloning of a nucleoside diphosphate kinase from Dictyostelium discoideum. J Biol Chem 265, $10012-10018$.

Lascu I and Gonin P. (2000). The catalytic mechanism of nucleoside diphosphate kinases. J Bioenerg and Biomemr 32, 237-246.

Lascu I, Schaertl S, Wang C, Sarger C, Giartosio A, Briand G, Lacombe ML and Konrad M. (1997). A point mutation of human nucleoside diphosphate kinase A found in aggressive neuroblastoma affects protein folding. J Biol Chem 272, 15599-15602.

Lascu I, Pop RD, Porumb H, Presecan E and Proinov I. (1983). Pig heart nucleoside diphosphate kinase. Phosphorylation and interaction with Cibacron blue 3GA. Eur J Biochem 135, 497-503.

Levit MN, Abramczyk BM, Stock JB and Postel EH. (2002). Interactions between Escherichia coli nucleoside-diphosphate kinase and DNA. J Biol Chem 277, 5163-5167.

Linck RW. (1973). Comparative isolation of cilia and flagella from the lamellibranch mollusk, Aequipecten irradians. J Cell Sci 12, 345-367.

Lombardi DJ. (2006). Commentary: nm23, a metastasis suppressor gene with a tumor suppressor gene aptitude? J Bioenerg Biomembr 38, 177-80. 
Lombardi D, Sacchi A, D'Agostino G and Tibursi G. (1995). The association of the Nm23-M1 protein and beta-tubulin correlates with cell differentiation. Exp Cell Res 217, 267-271.

Luo Y, Vassilev PM, Li X, Kawanabe Y and Zhou J. (2003). Native polycystin 2 functions as a plasma membrane $\mathrm{Ca} 2+$ - permeable cation channel in renal epithelia. Mol Cell Biol 23, 2600-2607.

Ma D, Xing Z, Liu B, Pedigo NG, Zimmer SG, Bai Z, Postel EH and Kaetzel DM. (2002). NM23-H1 and NM23-H2 repress transcriptional activities of nuclease hypersensitive elements in the platelet-derived growth factor-A promoter. $J$ Biol Chem 277, 1560-1567.

MacDonald NJ, De La Rosa A, Benedict MA, Freije JM, Krutsch H and Steeg PS. (1993). A serine phosphorylation of $\mathrm{Nm} 23$, and not its nucleoside diphosphate kinase activity, correlates with suppression of tumor metastatic potential. $J$ Biol Chem 268, 25780-25789.

Marshall WF and Rosenbaum JL. (2001). Intraflagellar transport balances continuous turnover of outer doublet microtubules: implications for flagellar length control. $J$ Cell Biol 155, 405-414.

Martinvalet D, Zhu P and Lieberman J. (2005). Granzyme A induces caspase independent mitochondrial damage, a required first step for apoptosis. Immunity 22, 355-370.

Melki R, Lascu I, Carlier MF and Veron M. (1992). Nucleoside diphosphate kinase does not directly interact with tubulin nor microtubules. Biochem Biophys Res 
Commun 187, 65-72.

Michaud EJ and Yoder BK. (2006). The primary cilium in cell signaling and cancer. Cancer Res 66, 6463-6467.

Milon L, Meyer P, Chiadmi M, Munier A, Johansson M, Karlsson A, Lascu I, Capeau J, Janin $\mathrm{J}$ and Lacombe ML. (2000). The human nm23-H4 gene product is a mitochondrial nucleoside diphosphate kinase. J Biol Chem 275, 14264-14272.

Miranda-Vizuete A, Tsang K, Yu Y, Jimenez A, Pelto-Huikko M, Flickinger CJ, Sutovsky P and Oko R. (2003). Cloning and developmental analysis of murid spermatid-specific thioredoxin-2 (SPTRX-2), a novel sperm fibrous sheath protein and autoantigen. J Biol Chem 278, 44874-44885.

Mitchell BF, Pederse LB, Feely M, Rosenbaum JL and Mitchell DR. (2005). ATP production in Chlamydomonas reinhardtii flagella by glycolytic enzymes. Mol Biol Cell 16, 4509-4518.

Mitchell KAP, Szabo G and Otero AS. (2008). Direct binding of cytosolic NDP kinases to membrane lipids is regulated by nucleotides. Submitted.

Mitchell KAP, Gallagher BC, Szabo G and Otero AS. (2004). NDP kinase moves into developing primary cilia. Cell Motil Cyto 59, 62-73.

Mizrahi A, Molshanski-Mor S, Weinbaum C, Zheng Y, Hirshberg M and Pick E. (2005). Activation of the phagocyte NADPH oxidase by Rac guanine nucleotide exchange factors in conjunction with ATP and nucleoside diphosphate kinase. $J$ Biol Chem 280, 3802-3811. 
Morera S, Chiadmi M, LeBras G, Lascu I and Janin J. (1995). Mechanism of phosphate transfer by nucleotide diphosphate kinase X-ray structures of a phospho-histidine form of the enzymes from Drosophila and Dictyostelium. Biochemistry 34, 11062-11070.

Morgan D, Eley L, Sayer J, Strachan T, Yates LM, Craighead AS and Goodship JA. (2002). Expression analyses and interaction with Apc2 suggest a role for inversin in primary cilia and in the cell cycle. Hum Mol Genet 11, 3345-3350.

Moynie' L, Giraud M-F, Georgescauld F, Lascu I and Dautant A. (2007). The structure of the escherichia coli nucleoside diphosphate kinase reveals a new quaternary architecture for this enzyme family. Prot. Struc. Func. Bioinform. 67, 755-765.

Munier A, Serres C, Kann ML, Boissan M, Lesaffre C, Capeau J, Fouquet JP and Lacombe ML. (2003). Nm23/NDP kinases in human male germ cells: role in spermiogenesis and sperm motility? Exp Cell Res 289, 295-306.

Nallamothu G, Woolworth JA, Dammai V and Hsu T. (2008). awd, the homolog of metastasis supressor gene Nm23, regulates Drosophila epithelial cell invasion. Mol Cell Biol 28, 1964-1973.

Nauli SM, Alenghat FJ, Luo Y, Williams E, Vassilev P, Li X, Elia AE, Lu W, Brown EM, Quinn SJ, Ingber DE and Zhou J. (2003). Polycystins 1 and 2 mediate mechanosensation in the primary cilium of kidney cells. Nat Genet 33, 129-137.

Nickerson JA and Wells WW. (1978). Association of nucleosidediphosphate kinase with microtubules. Biochem Biophys Res Commun 85, 820-826.

Novikoff AB, Shin WY and Drucker J. (1961). Mitochondrial localization of oxidative 
enzymes: staining results with two tetrazolium salts. J Biophys Biochem Cytol. 9, $47-61$.

Oda H, Stockert RJ, Collins C, Wang H, Novikoff PM, Satir P and Wolkoff AW. (1995). Interaction of the microtubule cytoskeleton with endocytic vesicles and cytoplasmic dynein in cultured rat hepatocytes. J Biol Chem 270, 15242-15249.

Orlov NY and Kimura N. (1998). Interaction of nucleoside diphosphate kinase with membranes of bleached bovine retinal rod outer segments. Effects of $\mathrm{pH}$, salts, and guanine nucleotides. Biochemistry (Mosc) 63, 171-179.

Ostrowski LE, Blackburn K, Radde KM, Moyer MB, Schlatzer DM, Moseley A and Boucher RC. (2002). A proteomic analysis of human cilia: identification of novel components. Mol Cell Proteomics 1, 451-465.

Otero AS. (2000). NM23/nucleoside diphosphate kinase and signal transduction. $J$ Bioenerg Biomembr 32, 269-275.

Otero AS, Doyle MB, Hartsough MT and Steeg PS. (1999). Wild- type NM23-H1, but not its S120 mutants, suppresses desensitization of muscarinic potassium current. Biochim Biophys Acta 1449, 157-168.

Otero AS. (1997). Copurification of vimentin, energy metabolism enzymes, and a MER5 homolog with nucleoside diphosphate kinase. Identification of tissue-specific interactions. J Biol Chem 272, 14690-14694.

Otsuki Y, Tanaka M, Yoshii S, Kawazoe N, Nakaya K and Sugimura H. (2001). Tumor metastasis suppressor nm23H1 regulates Rac1 GTPase by interaction with Tiam1. Proc Natl Acad Sci USA 98, 4385-4390. 
Ouatas T, Abdallah B, Gasmi L, Bourdais J, Postel E and Mazabraud A. (1997). Three different genes encode NM23/nucleoside diphosphate kinases in Xenopus laevis. Gene 194, 215-225.

Palacios F, Schweitzer JK, Boshans RL and D'Souza-Schorey C. (2002). ARF6-GTP recruits Nm23-H1 to facilitate dynamin-mediated endocytosis during adherens junctions disassembly. Nat Cell Biol 4, 929-936.

Pan J and Snell WJ. (2000). Regulated targeting of a protein kinase into an intact flagellum. An aurora/Ipl1p-like protein kinase translocates from the cell body into the flagella during gamete activation in Chlamydomonas. J Biol Chem 275, 24106-24114.

Parks RE and Agarwal RP. (1973). Nucleoside Diphosphokinases, in: PD Boyer, (Ed.), The Enzymes, Vol. VIII, Academic Press, NY, pp. 307-333.

Pazour GJ and Witman GB. (2003). The vertebrate primary cilium is a sensory organelle. Curr Opin Cell Biol 15, 105-110.

Pazour GJ and Rosenbaum JL. (2002). Intraflagellar transport and cilia-dependent diseases. Trends Cell Biol 12, 551-555.

Pazour GJ, San Agustin JT, Follit JA, Rosenbaum JL and Witman GB. (2002). Polycystin-2 localizes to kidney cilia and the ciliary level is elevated in orpk mice with polycystic kidney disease. Curr Biol 12, R378-R380.

Pfeffer SR and Kelly RB. (1981). Identification of minor components of coated vesicles by use of permeation chromatography. J Cell Biol 91, 385-391.

Phang-Ba V, Munier A, Lascu I, Lajarte-Thirouard A-S, PreÂvot S, Capeau J and 
Lacombe ML. (1998). Preparation of specific polyclonal antibodies against protein isoforms: Application to nucleoside diphosphate kinase A, Science Tools from Amersham Pharmacia Biotech 3, 10-11.

Pinon VP, Millot G, Munier A, Vassy J, Linares-Cruz G, Capeau J, Calvo F and Lacombe ML. (1999). Cytoskeletal association of the A and B nucleoside diphosphate kinases of interphasic but not mitotic human carcinoma cell lines: specific nuclear localization of the B subunit. Exp Cell Res 246, 355-367.

Piperno G, LeDizet M and Chang XJ. (1987). Microtubules containing acetylated alphatubulin in mammalian cells in culture. $J$ Cell Biol 104, 289-302.

Piperno G and Fuller MT. (1985). Monoclonal antibodies specific for an acetylated form of alpha-tubulin recognize the antigen in cilia and flagella from a variety of organisms. J Cell Biol 101, 2085-2094.

Poole CA, Flint MH and Beaumont BW. (1985). Analysis of the morphology and function of primary cilia in connective tissues: a cellular cybernetic probe? Cell Motil 5, 175-193.

Postel EH, Steven JB, Rooney JW and Kaetzel DM. (2000). Human NM23/nucleoside diphosphate kinase regulates gene expression through DNA binding to nucleasehypersensitive transcriptional elements. J Bioenerg Biomembr 32, 277-284.

Postel EH and Ferrone CA. (1994). Nucleoside diphosphate kinase enzyme activity of $\mathrm{NM} 23-\mathrm{H} 2 / \mathrm{PuF}$ is not required for its DNA binding and in vitro transcriptional functions. J Biol Chem 269, 9627-9630.

Praetorius HA and Spring KR. (2005). A physiological view of the primary cilium. Annu 
Rev Physiol 67, 515-529.

Praetorius HA and Spring KR. (2003). Removal of the MDCK cell primary cilium abolishes flow sensing. $J$ Membr Biol 191, 69-76.

Praetorius HA and Spring KR. (2001). Bending the MDCK cell primary cilium increases intracellular calcium. $J$ Membr Biol 184, 71-79.

Qin H, Diener DR, Geimer S, Cole DG and Rosenbaum JL. (2004). Intraflagellar transport (IFT) cargo: IFT transports flagellar precursors to the tip and turnover products to the cell body. J Cell Biol 164, 255-266.

Raveh S, Vinh J, Rossier J, Agou F and Veron M. (2001). Peptidic determinants and structural model of human NDP kinase B (Nm23-H2) bound to single-stranded DNA. Biochem 40, 5882-5893.

Rayner K, Chen Y-X, Hibbert B, White D, Miller H, Postel EH and O'Brien ER. (2008). Discovery of NM23-H2 as an estrogen receptor beta-associated protein:Role in estrogen-induced gene transcription and cell migration. J Steroid Biochem Mol Biol 108, 72-81.

Rayner K, Chen Y-X, Hibbert B, White D, Miller H, Postel EH and O'Brien ER. (2007). NM23-H2, an estrogen receptor beta-associated protein, shows diminished expression with progression of atherosclerosis. Am J Physiol Reg Int Comp Physiol 292, 743-750.

Robbe K and Antonny B. (2003). Liposomes in the study of GDP/GTP cycle of Arf and related small G proteins, Meth Enzymol 372, 151-166. 
Roberts RL, Barbieri MA, Ullrich J and Stahl PD. (2000). Dynamics of rab5 activation in endocytosis and phagocytosis. J Leukoc Biol 68, 627-32.

Robinson JB, Brems DN and Stellwagen E. (1981). A monoisozymic nucleoside diphosphate kinase capable of complete phosphorylation. J Biol Chem 256, 10769-10773.

Rochdi MD, Laroche G, Dupre E, Giguere P, Lebel A, Watier V, Hamelin E, Lepine MC, Dupuis G and Parent JL. (2004). Nm23-H2 interacts with a G proteincoupled receptor to regulate its endocytosis through an Rac-1 dependent mechanism. $J$ Biol Chem 279, 18981-18989.

Rosenbaum JL and Witman GB. (2002). Intraflagellar transport. Nat Rev Mol Cell Biol 3, $813-825$.

Rosenbaum JL and Child FM. (1967). Flagellar Regeneration in Protozoan Flagellates. $J$ Cell Biol 34, 345-364.

Roymans D, Willems R, Van Blockstaele DR and Slegers H. (2002). Nucleoside diphosphate kinase (NDPK/NM23) and the waltz with multiple partners: Possible consequences in tumor metastasis. Clin Exp Metas 19, 465-476.

Roymans D, Vissenberg K, De Jonghe C, Willems R, Engler G, Kimura N, Grobben B, Claes P, Verbelen JP, Van Broeckhoven C and Slegers H. (2001). Identification of the tumor metastasis suppressor Nm23-H1/Nm23-R1 as a constituent of the centrosome. Exp Cell Res 262, 145-153.

Roymans D, Willems R, Vissenberg K, De Jonghe C, Grobben B, Claes P, Lascu I, Van Bockstaele D, Verbelen JP, Van Broeckhoven C and Slegers H. (2000). 
Nucleoside diphosphate kinase B (Nm23-R1/NDPKB) is associated with intermediate filaments and becomes upregulated upon camp-induced differentiation of rat C6 glioma. Exp Cell Res 261, 127-138.

Sadek CM, Jimenez A, Damdimopoulos AE, Kieselbach T, Nord M, Gustafsson JA, Spyrou G, Davis EC, Oko R, van der Hoorn FA and Miranda-Vizuete A. (2003). Characterization of human thioredoxin-like 2. A novel microtubule-binding thioredoxin expressed predominantly in the cilia of lung airway epithelium and spermatid manchette and axoneme. J Biol Chem 278, 13133-13142.

Sadek CM, Damdimopoulos AE, Pelto-Huikko M, Gustafsson JA, Spyrou G and Miranda-Vizuete A. (2001). Sptrx-2, a fusion protein composed of one thioredoxin and three tandemly repeated NDP-kinase domains is expressed in human testis germ cells. Genes Cells 6, 1077-1090.

Satir P and Christensen ST. (2008). Structure and function of mammalian cilia. Histochem Cell Biol 129, 687-693.

Satir P and Christensen ST. (2007). Overview of structure and function of mammalian cilia. Annu Rev Physiol 69, 377-400.

Sato K and Nakano A. (2007). Mechanisms of COPII vesicle formation and protein sorting. FEBS Lett 581, 2076-2082.

Schaertl S, Konrad M and Geeves MA. (1998). Substrate specificity of human nucleoside-diphosphate kinase revealed by transient kinetic analysis. J Biol Chem 273, 5662-5669.

Schneider L, Clement CA, Teilmann SC, Pazour GJ, Hoffmann EK, Satir P and 
Christensen ST. (2005). PDGFRalphaalpha signaling is regulated through the primary cilium in fibroblasts. Curr Biol 15, 1861-1866.

Schneider B, Norda A, Karlsson A, Veron M and Deville-Bonne D. (2002). Nucleotide affinity for a stable phosphorylated intermediate of nucleoside diphosphate kinase. Protein Sci 11, 1648-1656.

Scholey JM. (2003). Intraflagellar transport. Annu Rev Cell Dev Biol 19, 423-443.

Schroer TA, Schnapp BJ, Reese TS and Sheetz MP. 1988. The role of kinesin and other soluble factors in organelle movement along microtubules, J Cell Biol 107, 17851792.

Schulz S, Handel M, Schreff M, Schmidt H and Hollt V. (2000). Localization of five somatostatin receptors in the rat central nervous system using subtype-specific antibodies. $J$ Physiol (Paris) 94, 259-264.

Schwoebel ED, Ho TH and Moore MS. (2002). The mechanism of inhibition of Ran dependent nuclear transport by cellular ATP depletion. $J$ Cell Biol 157, 963-974.

Seong H-A, Jung H and Ha1 H. (2007). NM23-H1 tumor suppressor physically interacts with serine-threonine kinase receptor-associated protein, a transforming growth factor-beta (TGF-beta) receptor-interacting protein, and negatively regulates tgfbeta signaling. J Biol Chem 282, 12075-12096.

Serre L, Vallée B, Bureaud N, Schoentgen F and Zelwer C. (1998). Crystal structure of the phosphatidylethanolamine-binding protein from bovine brain: a novel structural class of phospholipid-binding proteins. Structure 6, 1255-1265. 
Sloboda RD. (2002). A healthy understanding of intraflagellar transport. Cell Motil Cytoskeleton 52, 1-8.

Song L and Dentler WL. (2001). Flagellar protein dynamics in Chlamydomonas. $J$ Biol Chem 276, 29754-29763.

Soroka CJ, Pate MK and Boyer JL. (1999). Canalicular export pumps traffic with polymeric immunoglobulin A receptor on the same microtubule-associated vesicle in rat liver. J Biol Chem 274, 26416-26424.

Srivastava S, Li Z, Ko K, Choudhury P, Albaqumi M, Johnson AK, Yan Y, Backer JM, Unutmaz D, Coetzee WA and Skolnik EY. (2006). Histidine phosphorylation of the potassium channel $\mathrm{KCa} 3.1$ by nucleoside diphosphate kinase $\mathrm{B}$ is required for activation of KCa3.1 and CD4 T cells. Mol Cell 24, 665-675.

Stahl JA, Leone A, Rosengard AM, Porter L, King CR and Steeg PS. (1991). Identification of a second human nm23 gene, nm23-H2. Cancer Res 51, 445-449.

Steeg PS and Theodorescu D. (2008). Metastasis: a therapeutic target for cancer. Nat Clin Pract Oncol 5, 206-219.

Steeg PS. (2004). Perspectives on classic article: metastasis suppressor genes. J Natl Cancer Inst 96, E4.

Steeg PS, Bevilacqua G, Kopper L, Thorgeirsson UP, Talmadge JE, Liotta LA and Sobel ME. (1988). Evidence for a novel gene associated with low tumor metastatic potential. J Natl Cancer Inst 80, 200-204.

Szabo, G. (1974). Dual mechanism for the action of cholesterol on membrane permeability. Nature. 252, 47-49 
Takahashi T, Matsushita K, Welsh MJ and Stokes JB. (1994). Effect of cAMP on intracellular and extracellular ATP content of C1- -secreting epithelia and 3T3 fibroblasts. J Biol Chem 269, 17853-17857.

Taulman PD, Haycraft CJ, Balkovetz DF and Yoder BK. (2001). Polaris, a protein involved in left-right axis patterning, localizes to basal bodies and cilia. Mol Biol Cell 12, 589-599.

Timmons L and Shearn A. (2000). Role of AWD/nucleoside diphosphate kinase in Drosophila development. J Bioenerg Biomembr 32, 293-300.

Tucker RW, Meade-Cobun KS, Jayaraman S and More NS. (1983). Centrioles, primary cilia and calcium in the growth of Balb/c $3 T 3$ cells. J Submicrosc Cytol 15, 139143.

van Meer G. (1998). Lipids of the Golgi membrane. Trends Cell Biol 8, 29-33.

Voelker DR. (2000). Interorganelle transport of aminoglycerophospholipids, Biochim Biophys Acta 1486, 97-107.

Wagner PD and Vu ND. (2000). Phosphorylation of geranyl and farnesyl pyrophosphates by NM23 proteins/nucleoside diphosphate kinases. J Biol Chem 275, 3557035576.

Wagner PD, Steeg PS and Vu ND. (1997). Two-component kinase-like activity of nm23 correlates with its motility-suppressing activity. Proc Natl Acad Sci USA 94, 9000-9005.

Wagner PD and Vu ND. (1995). Phosphorylation of ATP-citrate lyase by nucleoside diphosphate kinase. J Biol Chem 270, 21758-21764. 
Watanabe T and Flavin M. (1976). Nucleotide-metabolizing enzymes in Chlamydomonas flagella, J Biol Chem 251, 182-192.

Watson MR and Hopkins JM. (1962). Isolated cilia from Tetrahymena pyriformis. Expl Cell Res 28, 280-295.

Wheatley DN and Bowser SS. (2000). Length control of primary cilia: analysis of monociliate and multiciliate PtK1 cells. Biol Cell 92, 573-582.

Wheatley DN, Wang AM and Strugnell GE. (1996). Expression of primary cilia in mammalian cells. Cell Biol Int 20, 73-81.

Wieland T. (2007). Interaction of nucleoside diphosphate kinase B with heterotrimeric G protein beta-gamma dimers: consequences on $\mathrm{G}$ protein activation and stability. Arch Pharm 374, 373-383.

Xu GM, Sikaneta T, Sullivan BM, Zhang Q, Andreucci M, Stehle T, Drummond I and Arnaout MA. (2001) Polycystin-1 interacts with intermediate filaments. $J$ Biol Chem 276, 46544-46552.

Xu L, Murphy JM and Otero AS. (1996). Participation of nucleoside- diphosphate kinase in muscarinic $\mathrm{K}+$ channel activation does not involve GTP formation. $J$ Biol Chem 271, 21120-21125.

Yi XB, Sweitzer NM and Otero AS. (1995). Neutralizing antibodies to nucleoside diphosphate kinase inhibit the enzyme in vitro and in vivo: evidence for two distinct mechanisms of activation of atrial currents by ATPgammaS. Biochim Biophys Acta 1310, 334-342. 
Yoder BK, Hou X and Guay-Woodford LM. (2002). The polycystic kidney disease proteins, polycystin-1, polycystin-2, polaris, and cystin, are co-localized in renal cilia. J Am Soc Nephrol 13, 2508-2516.

Yoon JH, Singh P, Lee DH, Quia J, Cai S, O’Connor TR, Chen Y, Shen B and Pfeifer GP. (2005). Characterization of the 3 ' $\rightarrow 5^{\prime}$ ' exonuclease activity found in human nucleoside diphosphate kinase 1 (NDK1) and several of its homologs. Biochem 44, 15774-15786.

Zambrano F, Fleischer S and Fleischer B. (1975). Lipid composition of the Golgi apparatus of rat kidney and liver in comparison with other subcellular organelles. Biochim Biophys Acta 380, 357-69.

Zhang Y, Ross EM and Snell WJ. (1991). ATP-dependent regulation of flagellar adenylylcyclase in gametes of Chlamydomonas reinhardtii. J Biol Chem 266, 22954-22959.

Zimmermann KW. (1898). Beiträge zur Kenntniss einiger Drüsen und epithelien. Arch Mikrosk Anat 52, 552-706. 


\title{
Receptor activation regulates cortical, but not vesicular localization of NDP kinase
}

\author{
Betty C. Gallagher, Kimberly A. Parrott, Gabor Szabo and Angela de S. Otero* \\ Department of Molecular Physiology and Biological Physics, University of Virginia Medical School, Charlottesville, VA \\ 22908, USA \\ *Author for correspondence (e-mail: ado2t@virginia.edu) \\ Accepted 23 April 2003 Journal of Cell Science 116, 3239-3250 @2003 The Company of Biologists Ltd doi:10.1242/jcs.00630
}

\begin{abstract}
Summary
We used immunofluorescence techniques to determine the localization of nucleoside diphosphate (NDP) kinase in NIH-3T3 fibroblasts. We found that cytoplasmic NDP kinase can be separated into two populations according to subcellular localization and response to extracellular stimuli. Specifically, within minutes of stimulation of resting fibroblasts with serum, growth factors or bombesin, a portion of NDP kinase becomes associated with membrane ruffles and lamellipodia. Another pool of NDP kinase accumulates independently of stimulation around intracellular vesicles. Transfection of cells with activated Rac mimics, whereas expression of dominant negative Rac inhibits, the effects of extracellular stimulation on the translocation of NDP kinase to the cell cortex. Neither Rac mutant affects the vesicle-associated pool. Association of NDP kinase with vesicles depends on microtubule integrity and is disrupted by nocodazole. In cell-free assays NDP kinase binds tightly to membrane vesicles associated with taxol-stabilized microtubules. Binding of NDP kinase to this fraction is reduced by ATP and abolished by GTP, as well as guanine nucleotides that are NDP kinase substrates. Thus, the localization of the two NDP kinase pools identified here is regulated independently by distinct cellular components: the appearance of cortical NDP kinase is a consequence of Rac activation, whereas vesicular NDP kinase is responsive to microtubule dynamics and nucleotides, in particular GTP. These results suggest that in fibroblasts NDP kinase participates in Racrelated cortical events and in GTP-dependent processes linked to intracellular vesicle trafficking.
\end{abstract}

Key words: NDP kinase, NM23, Microtubules, Rac

\section{Introduction}

The NM23/NDP kinase gene family encodes a group of eight homologous proteins with conserved structure. In higher organisms NDP kinases A and B are the most abundant members of the group and they are the best characterized to date (Lacombe et al., 2000). The bulk of mammalian NDP kinase A and B are cytosolic and form stable heterohexamers that can only be resolved into homogeneous preparations of NDP kinase A and B polypeptide chains after denaturation (Gilles et al., 1991). Using ATP as a phosphate donor for the synthesis of other nucleotides such as GTP, UTP and CTP, NDP kinases play an important role in the synthesis of nucleic acids and proteins, as well as in the metabolism of sugars and lipids (Lacombe et al., 2000). In recent years, it has become apparent that changes in the expression levels or modifications in the structure of NDP kinases alter functions as diverse as development, cell migration and differentiation in unexpected ways, leading to the suggestion that NDP kinases are multifunctional proteins (Otero, 2000; Kimura et al., 2000; Postel et al., 2002). For instance, members of this family were reported to inhibit growth-factor-induced cell motility of breast cancer cells (Kantor et al., 1993), attenuate the desensitization 
of muscarinic-activated atrial $\mathrm{K}^{+}$channels (Xu et al., 1996; Otero et al., 1999) and regulate synaptic vesicle114 internalization in Drosophila (Krishnan et al., 2001).

At the molecular level, the finding that NM23-H1 (human NDP kinase A) interacts with Tiam1, a Rac guanine nucleotide exchange factor, and downregulates Tiam1-Rac1 signaling, implied that it could affect remodeling of the actin cytoskeleton (Otsuki et al., 2001). More recently, Palacios et al. demonstrated that constitutively activated ARF6 binds NM23H1 and recruits NM23-H1 to cell junctions (Palacios et al., 2002). The presence of NM23-H1 at these sites facilitates dynamin-dependent endocytosis and downregulates Rac1 activity. Additionally, NM23-H2 (human NDP kinase B) was found to be linked to $\beta$-integrins through integrin cytoplasmic domain associated protein 1- $\alpha$ (ICAP1- $\alpha$ ) (Fournier et al., 2002), which inhibits activation of Rac1 and Cdc42 GTPases during integrin-mediated cell adhesion (Degani et al., 2002). Taken together, these reports hint at the multiple ways by which NDP kinases A and B could affect cell adhesion, signaling and motility. One important question is whether activation of surface receptors that trigger signal transduction pathways can modulate the subcellular distribution of the essentially cytosolic NDP kinases A and B in a manner that is spatially and temporally consistent with a role in signaling. In the present study, we examined the ability of NDP kinases A and B to respond to activation of receptor tyrosine kinases and G-protein-coupled receptors (GPCRs) by monitoring their spatial distribution in NIH-3T3 fibroblasts. These experiments allowed us to identify two distinct pools of NDP kinase in these cells: one population is rapidly translocated to the cell periphery when receptors are activated, whereas a second pool binds constitutively to microtubule-associated vesicles.

Vesicular NDP kinase is released by nucleotides, with GTP being more efficient than ATP; this suggests that NDP kinase might associate with intracellular vesicles when GTP levels are low, in order to provide the substrate used by the many GTPases that control intracellular trafficking.

\section{Materials and Methods \\ Materials}

Epidermal growth factor (EGF) and nucleotides were from Roche Molecular Biochemicals. Rhodamine-labeled phalloidin, Taxol, nocodazole, bombesin, 2,3-butanedione monoxime (BDM) and platelet-derived growth factor (PDGF) were from Sigma-Aldrich. Texas Red dextran (lysine-fixable, 70,000 Mr) was from Molecular Probes. The expression plasmid for N-terminal 3x -hemagglutinin (HA)-tagged human Rac1 (G12V and T17N mutants) was from the Guthrie cDNA Resource Center (Sayre, PA); the green fluorescent protein (GFP) vector pGreen Lantern was from Life Technologies. Gradient SDS-PAGE gels were from BioRad.

The monoclonal antibody specific for NDP kinase A (NM301) was from Santa Cruz Biotechnology; this antibody does not recognize NDP kinase B. Immunofluorescence staining of NDP kinase was also performed with a polyclonal antibody, Ab-1 (Labvision, Freemont, CA), raised to a homologous inner region of human NDP kinases A and B (amino acids 86-102). This antibody reacts with two proteins with the relative mobility of NDP kinase $\mathrm{A}$ and $\mathrm{B}$ in immunoblots of lysates from NIH-3T3 cells; no cross-reactivity with other proteins was detected (not shown). Although Ab-1 yielded similar results to NM301, it often stained cell nuclei, in agreement with the finding that NDP kinase B can localize to the nucleus (Kraeft et al., 1996; Pinon et al., 1999; Barraud et al., 2002). Like other rodent cells (Kimura et al., 2000; Barraud et al., 2002), NIH-3T3 fibroblasts express much higher levels of NDP kinase B than NDP kinase A (not shown). Therefore, the signal obtained with Ab-1 is presumably dominated by the B isoform. NDP kinase was detected in immunoblots with a rabbit polyclonal antibody that recognizes mammalian NDP kinases A and B (a generous gift from I. Lascu, Université de Bordeaux). Other antibodies used were: polyclonal anti-Rab4 (StressGen), polyclonal anti-Tiam1 (Santa Cruz), monoclonal anti-Rac (clone 23A8, Upstate Biotechnology) monoclonal anti-HA epitope tag (12CA5, Exalpha), monoclonals anti-LAMP-1, anti-kinesin heavy chain and $\mathrm{Na}^{+} \mathrm{K}^{+}$-ATPase (1D4B, SUK4 and $\alpha$, respectively, from the Developmental Studies Hybridoma Bank, Iowa) and monoclonal anti- $\alpha$-tubulin (clone DM1A, Sigma-Aldrich). Texas Red-transferrin and all secondary antibodies (Texas Red or Cy2 conjugates, multiple labeling grade) were from Jackson Immunoresearch Laboratories. Specificity of immunofluorescence labeling was established by pre-incubation with excess antigen, which effectively blocked cell staining. The secondary antibodies did not stain cells in the absence of primary antibodies.

Cell culture and transfection

NIH-3T3 fibroblasts were grown in Dulbecco's modified Eagle's medium supplemented with $10 \%$ fetal bovine serum, $2 \mathrm{mM} \mathrm{L-glutamine,} 1 \mathrm{mM}$ sodium pyruvate, $100 \mathrm{U} \mathrm{l}^{-1}$ penicillin and $100 \mu \mathrm{g} \mathrm{ml}^{-1}$ streptomycin at $37^{\circ} \mathrm{C}$ in a 
humidified 5\% $\mathrm{CO}_{2}$ atmosphere. Cells were subcultured at 50-70\% confluence; assays were performed on cultures 115 derived from the same stock, between passages 2 and 8 . Transfections were performed using LipofectAMINE ${ }^{\mathrm{TM}}$ 2000 as recommended by the manufacturer.

\section{Immunofluorescence}

Cells were grown on No. 1 thickness acid-washed glass coverslips. For experiments, cells 30-40\% confluent were placed in serum-free medium for 16-24 hours and then treated with $10 \mathrm{ng} \mathrm{ml}^{-1}$ EGF, $10 \mathrm{nM}$ bombesin or $10 \%$ serum for the times specified in the figure legends. Treatment with $30 \mathrm{mM}$ BDM was performed in serum-free medium supplemented with $0.5 \%$ bovine serum albumin (BSA) and $10 \mathrm{mM}$ glucose; cells were then stimulated with EGF for 2 minutes.

Cells were fixed for 10 minutes at room temperature in $4 \%$ paraformaldehyde $/ 0.1 \%$ glutaraldehyde. Free aldehyde groups were quenched with $\mathrm{NaBH}_{4}$, and cells were then stained as described (Pinon et al., 1999). Briefly, after a wash with PBS, cells were transferred to a blocking solution composed of $0.1 \%$ saponin and $3 \%$ BSA in PBS. This same buffer was used to dilute primary and secondary antibodies. Coverslips were incubated overnight with primary antibodies at $4^{\circ} \mathrm{C}$, washed extensively with PBS and incubated for 45 minutes at room temperature with secondary antibodies. After washes with PBS, coverslips were mounted in Mowiol (Calbiochem) containing 2\% $n$-propylgallate. To stain F-actin, rhodamine-conjugated phalloidin $\left(0.05 \mathrm{ng} \mathrm{ml}^{-1}\right)$ was added to the secondary antibody solution. The coverslips were examined on a Nikon Diaphot microscope equipped with a $40 \times$ oil immersion objective. Digital images were obtained with a Nikon CoolPix 990 camera. The phase-contrast images of the immunolabeled cells were routinely collected to confirm the identification of specific cell features. Figures were assembled using Adobe Photoshop software. Results shown are representative of 4-25 independent experiments.

For fluorescence ratio imaging, NIH-3T3 cells transfected with GFP were fixed and immunostained with NM301 followed by Texas-Red-conjugated anti-mouse IgG. Paired images of GFP and Texas Red fluorescence were acquired under conditions designed to avoid pixel saturation, inspected to verify alignment and saved as TIFF (8-bit) files. Ratio images (NDP kinase A/GFP) were obtained with Scion Image (Scion). Line profiles obtained with Maxim DL 2.12 (Diffraction Limited) were used for quantitative analy sis of fluorescence values in digitized images.

Measurements of the diameters of NDP-kinase-labeled vesicles were performed in images of cells immunolabeled with Ab-1 or NM301. Histograms were constructed using a bin size of $0.5 \mu \mathrm{m}$.

\section{Subcellular fractionation Membrane preparation}

Isolation of the particulate fraction from quiescent and serum stimulated cells was performed by the method of Del Pozo et al. (Del Pozo et al., 2002) with slight modifications. Briefly, serum-deprived cells in $10 \mathrm{~cm}$ dishes were treated with medium containing $0 \%$ or $10 \%$ serum for 10 minutes, rinsed with PBS and incubated in ice-cold lysis buffer (10 mM Tris, pH 7.4 with $\mathrm{HCl}, 1.5 \mathrm{mM} \mathrm{MgCl} 2,5 \mathrm{mM} \mathrm{KCl}, 1 \mathrm{mM}$ DTT, $0.2 \mathrm{mM}$ sodium vanadate, $1 \mathrm{mM}$ PMSF, $1 \mu \mathrm{g} \mathrm{ml}^{-1}$ each aprotinin and leupeptin) for 5 minutes. Cells were scraped, homogenized by 15 passes in a Dounce homogenizer and the lysates were centrifuged at $700 \mathrm{~g}$ for 3 minutes. The supernatants were spun for 15 minutes at 167,000 $\mathrm{g}$ in a Beckman Airfuge; the cytosolic fraction was removed, the membrane pellet was washed once with lysis buffer. Samples containing equal amounts of protein were solubilized in SDS-PAGE sample buffer with $20 \mathrm{mM}$ DTT, alkylated with $60 \mathrm{mM}$ iodoacetamide and resolved in $15 \%$ or $4-20 \%$ minigels. Proteins were transferred to nitrocellulose and immunoblotted with antibodies to Rac and NM23 as described previously (Otero, 1997). Immunoblotting with the plasma membrane marker $\mathrm{Na}^{+}, \mathrm{K}^{+}$-ATPase was used to verify equal loading of control and serum-treated samples. Results are expressed as the proportion of the protein detected in control membrane fractions. Proteins were detected by chemiluminescence and quantified using Scion Image.

\section{Isolation of microtubule-associated endocytic vesicles and proteins}

Endocytic vesicles associated with endogenous microtubules were isolated essentially as described by Wolkoff and colleagues (Goltz et al., 1992; Oda et al., 1995). Briefly, $80 \%$ confluent cells in $15 \mathrm{~cm}$ plates were washed with PBS, scraped into $0.6 \mathrm{ml}$ MEPS buffer (35 mM PIPES pH

7.1 with $\mathrm{NaOH}, 5 \mathrm{mM} \mathrm{MgSO} 4,5 \mathrm{mM}$ EGTA, $200 \mathrm{mM}$ sucrose, $1 \mathrm{mM}$ DTT) containing protease inhibitors (2 mM PMSF, $1 \mathrm{mM}$ benzamidine, $2 \mu \mathrm{g} \mathrm{ml}^{-1}$ leupeptin) and homogenized by 10-14 passes through a $27 \mathrm{G}$ needle. Homogenates were centrifuged at $1000 \mathrm{~g}$ for 10 minutes to sediment nuclei and large debris. The post-nuclear supernatant was centrifuged at $40,000 \mathrm{~g}$ for 20 minutes. The supernatant (small vesicles and cytosol) was incubated at $37^{\circ} \mathrm{C}$ for 30 minutes with $20 \mu \mathrm{M}$ Taxol (and nucleotides when indicated) to polymerize tubulin. The resulting microtubules and associated vesicles were pelleted at $16,000 \mathrm{~g}$ for 30 minutes at $4^{\circ} \mathrm{C}$. The pellets were brought to the original volume with MEPS, taxol was added and the samples were centrifuged at 16,000 $\mathrm{g}$. After a 
second wash, pellets were solubilized in SDSPAGE sample buffer and analysed as described above. Results shown116 are representative of 3-12 separate experiments .

To determine whether NDP kinase is initially associated with vesicles or soluble tubulin, the supernatant of the $40,000 \mathrm{~g}$ step was centrifuged at $230,000 \mathrm{~g}$ for 1 hour in a TLA 100.3 rotor (Beckman Coulter) to sediment membrane vesicles. The supernatant was incubated with taxol as described above to polymerize soluble tubulin, and centrifuged at 16,000 $\mathrm{g}$. Both pellets were washed twice with MEPS and analyzed by immunoblotting .

\section{Results}

NDP kinase is associated dynamically with membrane ruffles and constitutively with intracellular vesicles
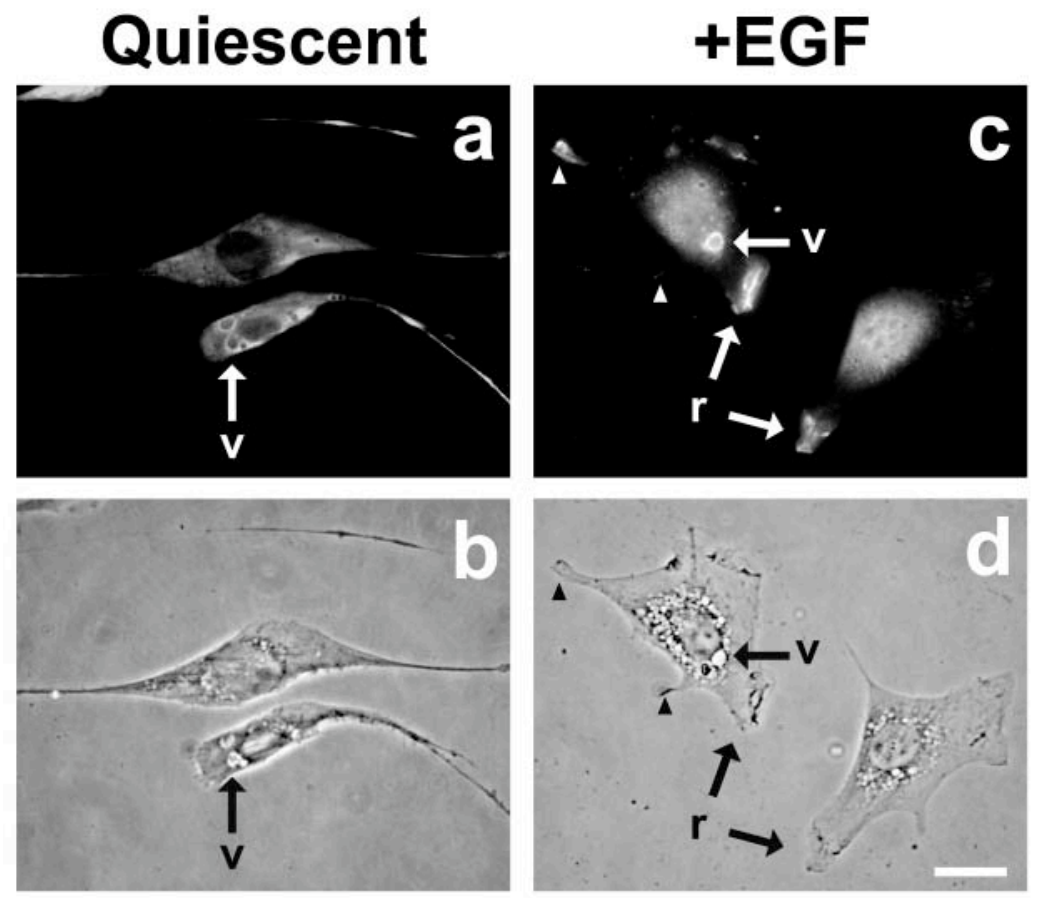

Fig. 1. Localization of NDP kinase in quiescent and EGF-treated NIH-3T3 cells .

(a) In resting cells, NDP kinase accumulates in ring-like structures (v) that correspond to phase-bright cytoplasmic vesicles (b). (c) In cells treated with $10 \mathrm{ng} \mathrm{ml}^{-1}$ EGF for 2 minutes, NDP kinase is present in lamellipodia and ruffles (r) as well as around cytoplasmic vesicles (v); (d) the corresponding phase-contrast image. Arrowheads indicate areas where the intensity of the fluorescence signal does not coincide with phase-dark areas. NDP kinase was visualized with polyclonal Ab-1. Scale bar, $25 \mu \mathrm{m}$.

In quiescent, serum-starved NIH-3T3 fibroblasts, staining of NDP kinase with an antibody that recognizes NDP kinases A and B (Ab-1) reveals a cytosolic distribution pattern, more intense in the thicker central area (Fig. 1a). In many cells, NDP kinase forms ring-like structures, and phase-contrast microscopy (Fig. 1b) shows that most of these rings correspond to phase-bright vesicles of various sizes that are scattered through the cytoplasm, particularly around the nucleus. NDP kinase is associated with the outer rim of these structures, and is most conspicuous around large perinuclear vesicles (Fig. 1a).

In cells treated with EGF, NDP kinases are present not only in the cytosol and in vesicular structures but also at well-defined areas of the cell cortex. Fig. 1c shows several features of NDP kinase localization in cells exposed to EGF for 2 minutes; similar results were obtained with $5 \mathrm{nM}$ PDGF and with $10 \%$ fetal bovine serum (not shown). In stimulated cells, NDP kinase staining is apparent in the submembranous space of cell protrusions such as ruffling lamellipodia; the large, phase-bright vesicles labeled by NDP 
kinase in quiescent cells are still prominent after stimulation with EGF (Fig. 1c,d). Translocation of NDP117 kinase to ruffles is a rapid and transient event, being evident within 1 minute of exposure to growth factors (Fig. 2) and becoming less pronounced after 30 minutes of continued stimulation (data not shown).

Areas heavily stained for NDP kinase were not always
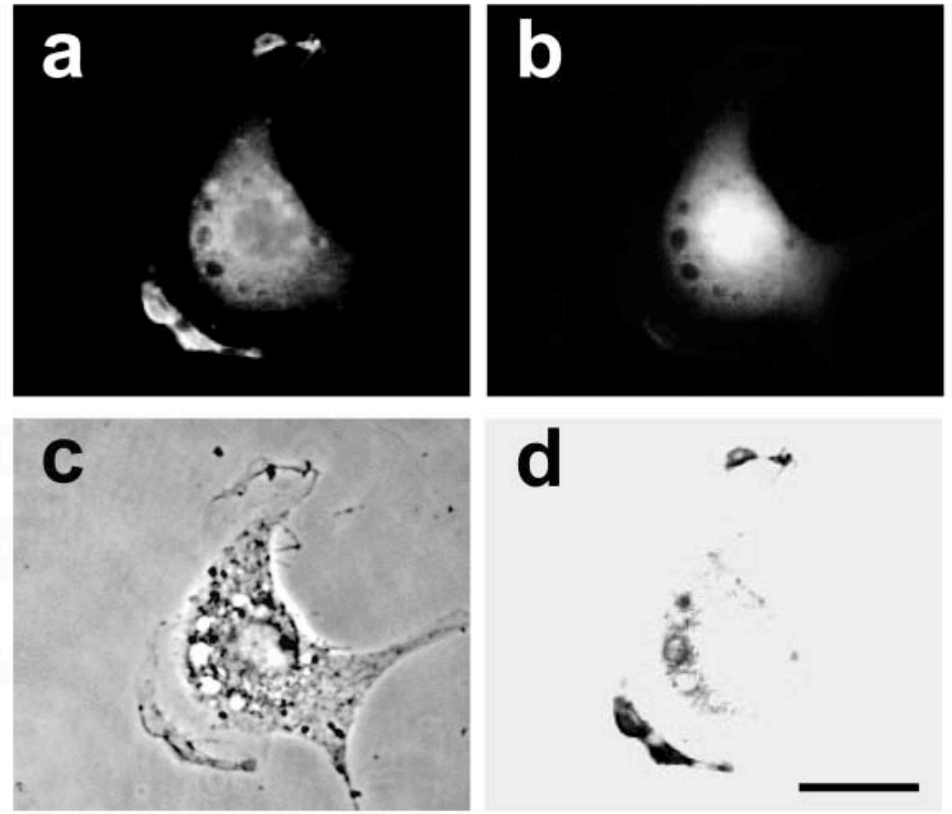

Fig. 2. The strong signal for NDP kinase around vesicles and in ruffling membranes is not a volume effect. Fluorescence ratio imaging of cells transfected with GFP and stained with anti-NDP kinase-A after treatment with $10 \mathrm{ng} \mathrm{ml}^{-1}$ EGF for 1 minute. (a) NDP kinase A, (b) GFP and (c) corresponding phase-contrast image. (d) Ratio image showing high NDP kinase A/GFP ratios (arbitrarily set as dark areas) at edge ruffles and around large vesicles in the perinuclear area. Scale bar, $25 \mu \mathrm{m}$.

phase dark; by the same token, NDP kinase did not accumulate in all phase-dense regions (Fig. 1c,d, arrowheads), suggesting that volume effects are not the main reason for its enrichment in specific areas. Nevertheless, given that NDP kinases A and B are abundant proteins, we tested the possibility that the increased fluorescence signal observed in ruffles and around vesicles reflected the increased thickness in these locations and not actual accumulation of the antigen. To measure the enrichment of NDP kinase around vesicles and in ruffles, we examined its distribution in cells transfected with GFP, a soluble protein that behaves as a volume marker for the cytosol (Kaksonen et al., 2000) and analysed the data by fluorescence ratio imaging. Fig. 2 shows a GFP-expressing cell stained with the antibody specific for NDP kinase A after a short (1 minute) exposure to EGF. Although the signal for both GFP and NDP kinase A is strong in the cell body, the two patterns are mostly distinct. NDP kinase A (Fig. 2a) is concentrated at ruffling lamellipodia and forms a ring around one of several perinuclear vesicles seen in the phase-contrast image (Fig. 2c). By contrast, GFP is not present in ruffles and is excluded from, but does not accumulate around, vesicles (Fig. 2b). The ratio image (Fig. 2d) illustrates the high ratios of NDP kinase A to GFP in the ruffling edge $(15: 1-20: 1)$ and at the rim of the vesicle seen in Fig. 2a $(2: 1-3: 1)$, and reveals a feature that is not obvious from the fluorescent image (Fig. 2a), namely that other vesicles are faintly positive for NDP kinase A. This analysis is consistent with a genuine enrichment of NDP kinase around vesicles as well as in ruffling membranes.

Comparison of Figs 1 and 2 shows that the monoclonal antibody specific to NDP kinase A and the polyclonal antibody that recognizes both NDP kinase A and B yield similar results, suggesting that these two isoforms coexist within the same areas of a cell. Therefore, in the following sections we refer to NDP kinase A and B jointly as NDP kinase. 

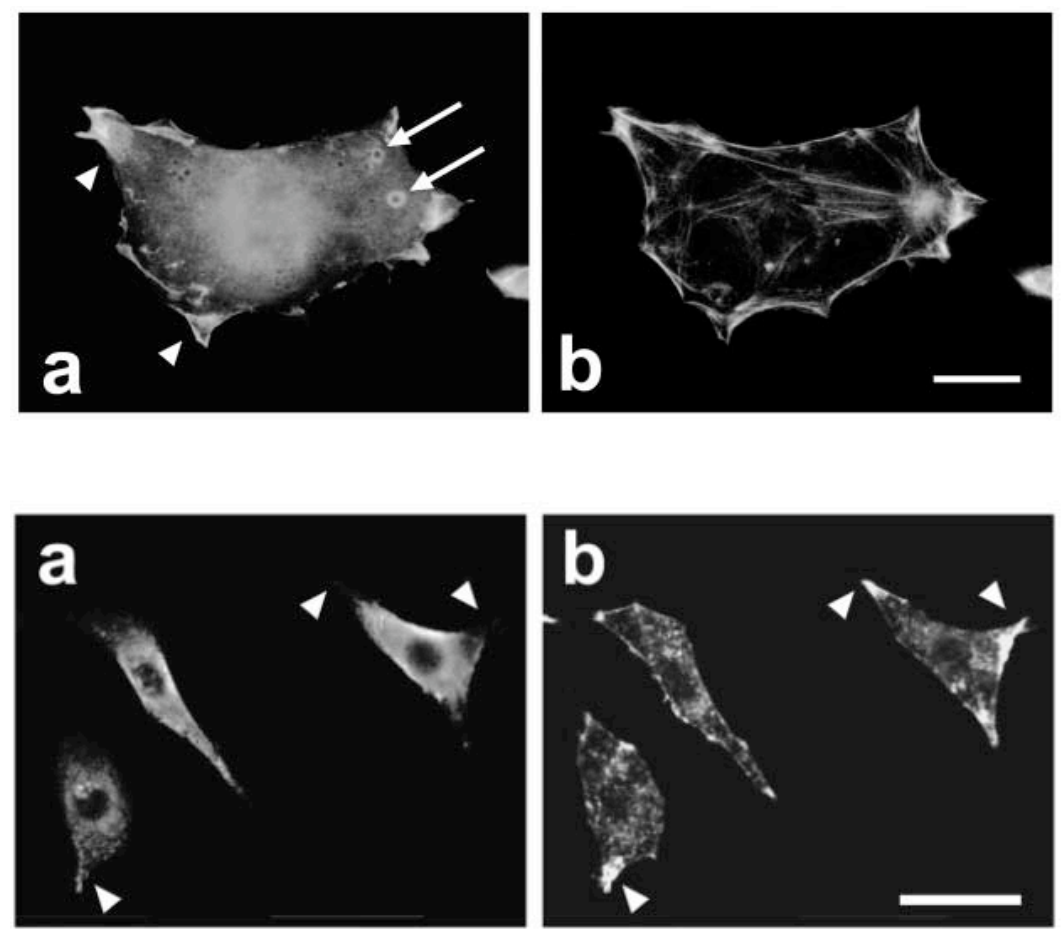

NDP kinase is translocated to ruffles in response to bombesin, a GPCR agonist

To determine whether the intracellular distribution of NDP kinase could be changed by stimulation of membrane receptors other than receptor tyrosine kinases (RTKs) such as the EGF and PDGF receptors, we also examined the localization of NDP kinase in cells stimulated by bombesin, which acts through a GPCR (Kjöller and Hall, 1999). Exposure of quiescent cells for 5-10 minutes to bombesin induced membrane ruffles that stained brightly for NDP kinase (Fig. 3a). Thus, stimulation of GPCRs, as well as RTKs, triggers the translocation of NDP kinase to the cell cortex.

Membrane ruffling results from profound changes in the organization of the actin cytoskeleton, with increased accumulation of F-actin at the cortex (Ridley et al., 1992). To determine whether the translocation of NDP kinase to ruffles is related to the redistribution of F-actin or actin binding proteins, we labeled stimulated cells with phalloidin and anti-NDP kinase. Fig. 3b shows the distribution of F-actin in a cell incubated with bombesin; similar results were obtained in cells treated with EGF. There is some overlap between NDP kinase staining and the intense F-actin signal at the edge of cells and at the tip of ruffles but the NDP kinase signal in ruffles typically forms broad patches that extend towards the center of the cell (Fig. 3a, arrowheads), well beyond the areas rich in F-actin. There is little or no co-localization of the two proteins elsewhere, with NDP kinase being absent from the stress fibers detected by phalloidin and the focal adhesions outlined by an antibody to vinculin (not shown). Notably, the vesicles labeled by NDP kinase antibodies (Fig. 3a, arrows) do not stain with phalloidin. In vitro studies with the purified proteins show no interaction between actin and NDP kinase (not shown). Thus, it appears that localization of NM23 to the cell periphery does not depend on a direct interaction with F-actin. To verify this hypothesis, we used BDM, which reduces ruffling (Rottner et

Fig. 3. Stimulation of cells with bombesin, a GPCR agonist, also induces translocation of NDP kinase to the actin-rich cell cortex. Serum-starved cells were treated with $10 \mathrm{nM}$ bombesin for 10 minutes, then stained with Ab-1 antibody (a) and rhodamine-phalloidin (b). Arrows show accumulation of NDP kinase around vesicles. The arrowheads indicate patches where NDP kinase staining extends beyond the actin-rich ruffling edge. Scale bar, 25 $\mu \mathrm{m}$. 
Fig. 4. BDM suppresses NDP kinase translocation to the cell cortex. Cells were pre-incubated with $30 \mathrm{mM}$ BDM for 30 minutes as described in Materials and Methods. The incubation was continued in the presence of $10 \mathrm{ng} \mathrm{ml}$ EGF for 2 minutes, followed by fixation and labeling with anti-NDP kinase A (a) and rhodamine-phalloidin (b). Notice the absence of NDP kinase staining in cell protrusions that remain enriched in F-actin (b, arrowheads). Scale bar, $50 \mu \mathrm{m}$. al., 1999) but does not interfere with actin polymerization (Cramer and Mitchison, 1995). When cells were treated with BDM prior to stimulation with EGF, NDP kinase remained in the cell body (Fig. 4a), whereas significant amounts of F-actin were observable at the cortex (Fig. 4b). BDM has no effect on the enzymatic activity of purified erythrocyte NDP kinase (B.

K.A.P. and A.d.S.O., unpublished). Taken together, these data imply that NDP kinase does not interact directly with F-actin and is translocated to the cell periphery through a mechanism distinct from binding to microfilaments. Given that BDM is a low-affinity myosin ATPase inhibitor, it is conceivable that a member of the large myosin family is involved on NDP kinase translocation. We are currently exploring this possibility.

Rac controls the translocation of NDP kinase to ruffles in response to activation of membrane receptors

Activation of Rac, a member of the Rho family of small GTPases that regulates the formation of lamellipodia and membrane ruffles, is a shared step in the response of fibroblasts to stimulation of different types of receptors (Kjöller and Hall, 1999). Our observations in cells responding to growth factors and bombesin suggest that localization of NDP kinase to the cell periphery is linked to a common event, possibly Rac activation. To test this hypothesis, we examined the distribution of NDP kinase in cells expressing a constitutively activated form of Rac1, RacG12V. Expression of RacG12V (Fig. 5a) induced extension of lamellipodia in unstimulated, serum-starved cells as expected (Ridley et al., 1992) and also promoted the appearance of NDP kinase at the periphery (Fig. 5b). By contrast, NIH-3T3 cells expressing the dominant negative Rac mutant RacT17N did not extend lamellipodia in response to EGF (Fig. 5d) and showed the perinuclear staining for NDP kinase typical of quiescent cells (Fig. 5e). The

\section{Dual localization of NDP kinase 3243}

numbers and appearance of NDP kinase-labeled intracellular vesicles were similar in cells expressing Rac mutants and in neighboring untransfected cells (Fig. 5b,e), indicating that Rac controls only the recruitment of NDP kinase to the cortex.

\section{Tiam1 is not involved in NDP kinase translocation}

Although NDP kinase and activated Rac co-localize in lamellipodia (Fig. 5a,b, arrow), NDP kinase does not bind directly to Rac (Otsuki et al., 2001) (K.A.P. and A.d.S.O., unpublished). Therefore, its connection to activated Rac is likely to be mediated by another cell component. NDP kinase A associates with a Rac1-specific nucleotide exchange factor, Tiam1 (Otsuki et al., 2001; Palacios et al., 2002). However, the idea of a Tiam1-mediated redistribution of NDP kinase to the cortex as a response to growth factors and bombesin is not supported by our results. Fig. 6 shows that there is limited overlap of the signals for NDP kinase A and Tiam1 at ruffles following exposure to EGF for 2 minutes. In particular, NDP kinase was visible throughout ruffling areas in cells, whereas Tiam1 staining was confined to small areas of the cell periphery (Fig. 6). This result is consistent with previous work showing that Tiam1's ability to translocate to membranes and to act as a GEF for Rac is markedly reduced in cells cultured in low or no serum media (Michiels et al., 1997; Bourguignon, 2000). Thus, under our experimental conditions, Tiam1 is not likely to be responsible for the translocation of NDP kinase to the cell cortex.

\section{Cell stimulation promotes association of NDP kinase with membranes}

The cortical staining of stimulated cells with NDP kinase antibodies might reflect an actual translocation from the

Fig. 5. Rac induces recruitment of NDP kinase to ruffles. Cells transfected with HA-tagged RacG12V (a,b,c) or 
RacT17N (d,e,f) were serum starved. Cells expressing RacG12V were fixed directly, whereas those expressing T17N Rac were first treated with EGF for 2 minutes. Samples were double labeled for the HA tag (a,d) and NDP kinase (b,e) with Ab-1. (c,f) Phase contrast images. In the cell that expresses activated Rac (a), NDP kinase is visible at the edge of the lamellipodium (b, arrow) and around vesicles (b, arrowheads). Expression of T17N Rac (d) inhibits accumulation of NDP kinase in ruffles in response to EGF (e; compare the transfected cell on the left with those on top right) but does not affect vesicular NDP kinase (e, arrowhead). Scale bar, $20 \mu \mathrm{m}$.
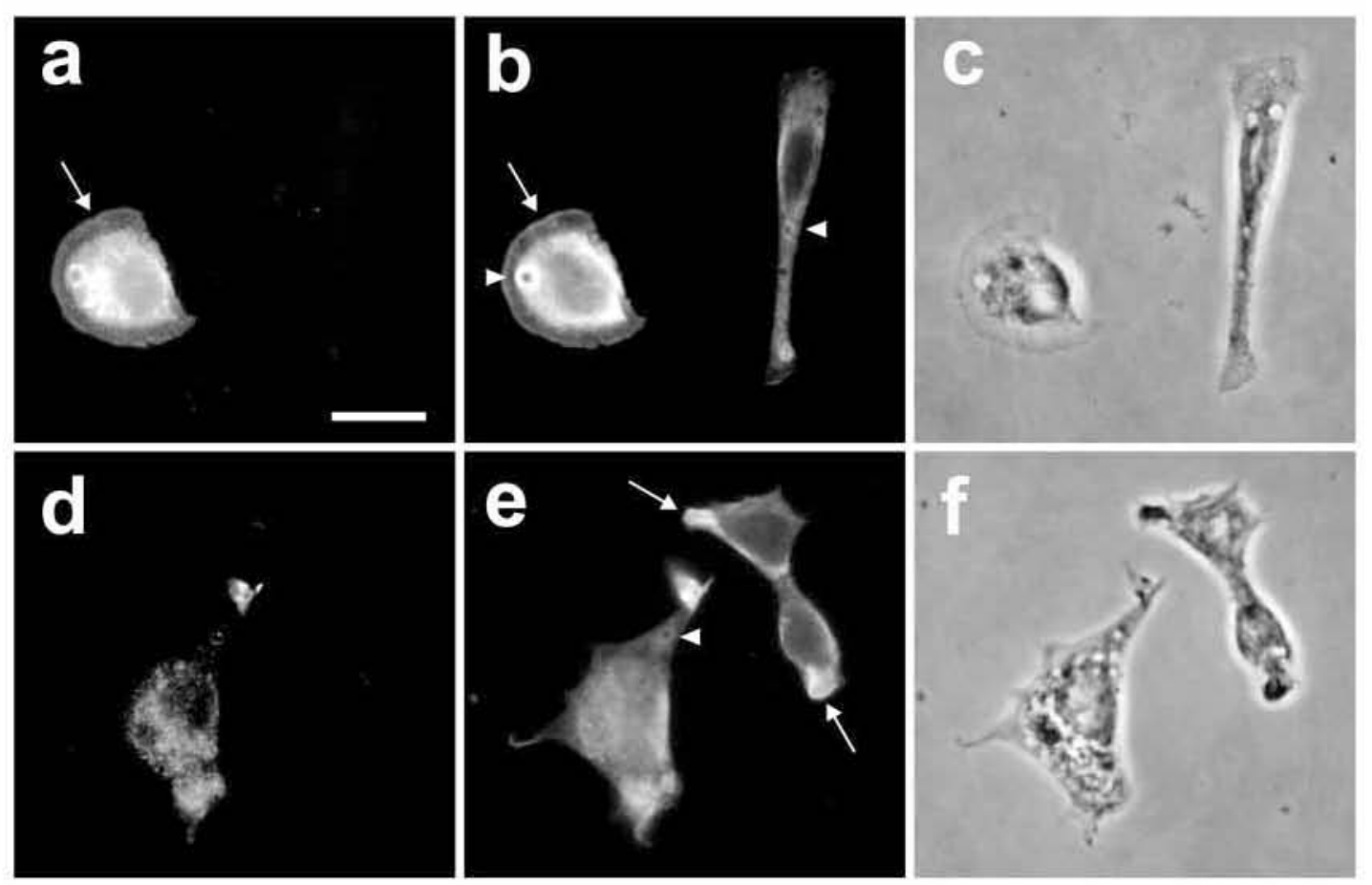

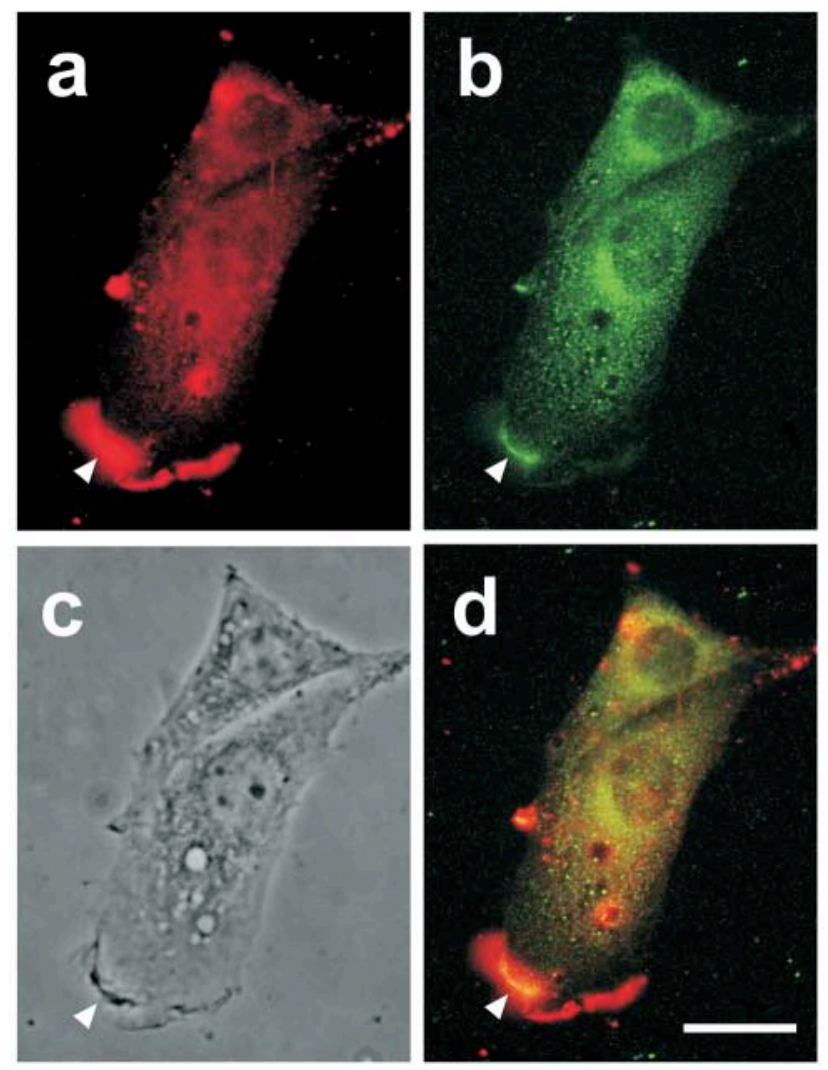

Fig. 6. Limited overlap of Tiam 1 and NDP kinase in ruffles. Serum-starved cells were treated with $10 \mathrm{ng} \mathrm{ml}^{-1} \mathrm{EGF}$ for 2 minutes. Cells were stained with anti-NDP kinase A (a) and anti-Tiam1 (b) antibodies; (c) phase contrast image. Notice that NDP kinase is present throughout the ruffle, whereas Tiam1 is confined to a small area (arrow). The merged image (d) highlights the limited overlap between the signals for Tiam1 and for NDP kinase. (c) Scale bar, $25 \mu \mathrm{m}$

cytosol to the plasma membrane, as observed with activated Rac. To test this hypothesis, we compared the distribution of Rac and NDP kinase by two independent approaches. First, we performed a line scan analysis of images of cells treated with EGF and fluorescently stained for endogenous Rac and NDP kinase. Fig. 7A shows that the fluorescence intensities for both NDP kinase and Rac are correlated only in peripheral ruffles. The two proteins do not co-localize elsewhere in the lamellipodium and, as seen also in Fig. 3b, the signal for NDP kinase, but not Rac, increases across a large patch immediately behind the ruffling region. Neither protein localizes to less active areas of the plasma membrane, indicating that the appearance of cortical NDP kinase is associated with ruffling activity. To confirm this result, we also compared the NDP kinase content of membrane fractions isolated from quiescent and serum-stimulated cells. Immunoblotting with antibodies to Rac and NDP kinase shows that the amounts of both proteins in the particulate fraction are similarly increased (3.5 times) following serum stimulation, whereas the plasma membrane marker $\mathrm{Na}^{+} \mathrm{K}^{+}$-ATPase remains unchanged, as expected (Fig. 7B).

Overall, these results demonstrate that, in NIH-3T3 cells, stimulation of surface receptors that lead to Rac activation 

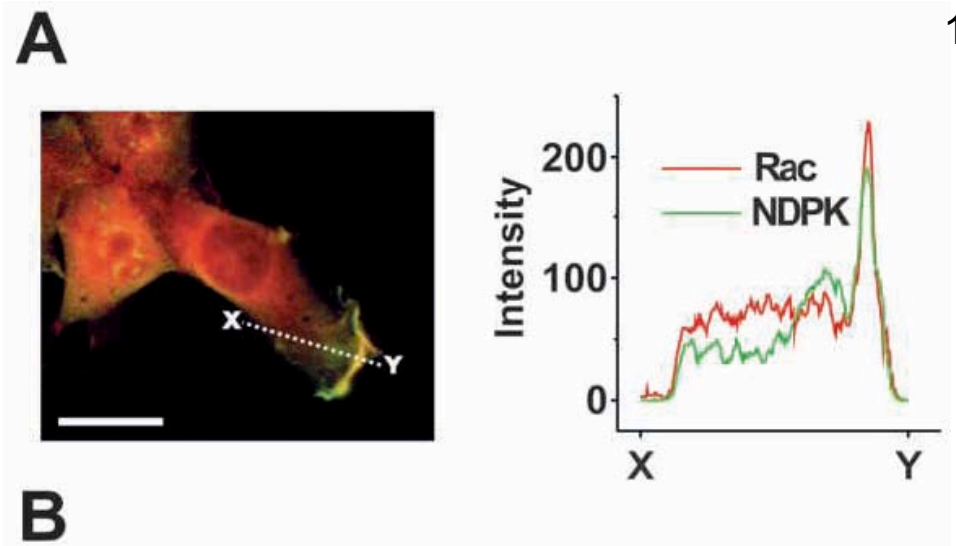

\section{NDPK}

\section{Rac}

\section{$\approx \mathrm{Na}^{+}, \mathrm{K}^{+}$- ATPase}

Fig. 7. Cell stimulation increases the association of NDP kinase and Rac with cell membranes. (A) Merged image of serum-starved cells treated with EGF for 2 minutes and stained for Rac (red) and NDP kinase (Ab-1; green); areas of overlap appear in yellow. The line scan analysis shows the fluorescence intensities of Rac and NDP kinase along the white line in the image. Scale bar, $25 \mu \mathrm{m}$. (B) Subcellular fractionation of quiescent and serum-treated cells was as in Methods. The particulate fraction was immunoblotted with antibodies to NDP kinase (NDPK), Rac and $\mathrm{Na}^{+}, \mathrm{K}^{+}$-ATPase, a plasma membrane marker.

triggers the translocation of a pool of NDP kinase to the cell periphery without affecting vesicular NDP kinase. The shift of NDP kinase towards the cell cortex is rapid, being evident within 1 minute of treatment with EGF, and results in extensive co-localization of NDP kinase and activated Rac at lamellipodia but does not involve a direct interaction with Rac, Tiam1 or F-actin.

\section{Characterization of the vesicles labeled by NDP kinase}

The results above show that the Rac-related events that lead to the dynamic targeting of NDP kinase to ruffles differ considerably from the biochemical processes responsible for its constitutive association with vesicles. To define more clearly the mechanism by which NDP kinase assembles around vesicles, we first characterized their morphology and protein composition. Fig. 8A shows the size distribution of NDP-kinase-labeled vesicles, which covers a range of 0.7-6.9 $\mu \mathrm{m}$ with a median diameter of $2.3 \mu \mathrm{m}$ $(n=209)$. There is no statistically significant difference $(P=0.19)$ between the size distribution of NDP-kinase-labeled vesicles in resting and stimulated cells. The large size and variable diameter of these vesicles, as well as lack of overlap of NDP kinase staining with internalized transferrin (not shown), indicate that they do not represent small, uniform sized clathrin-coated pits. Although 

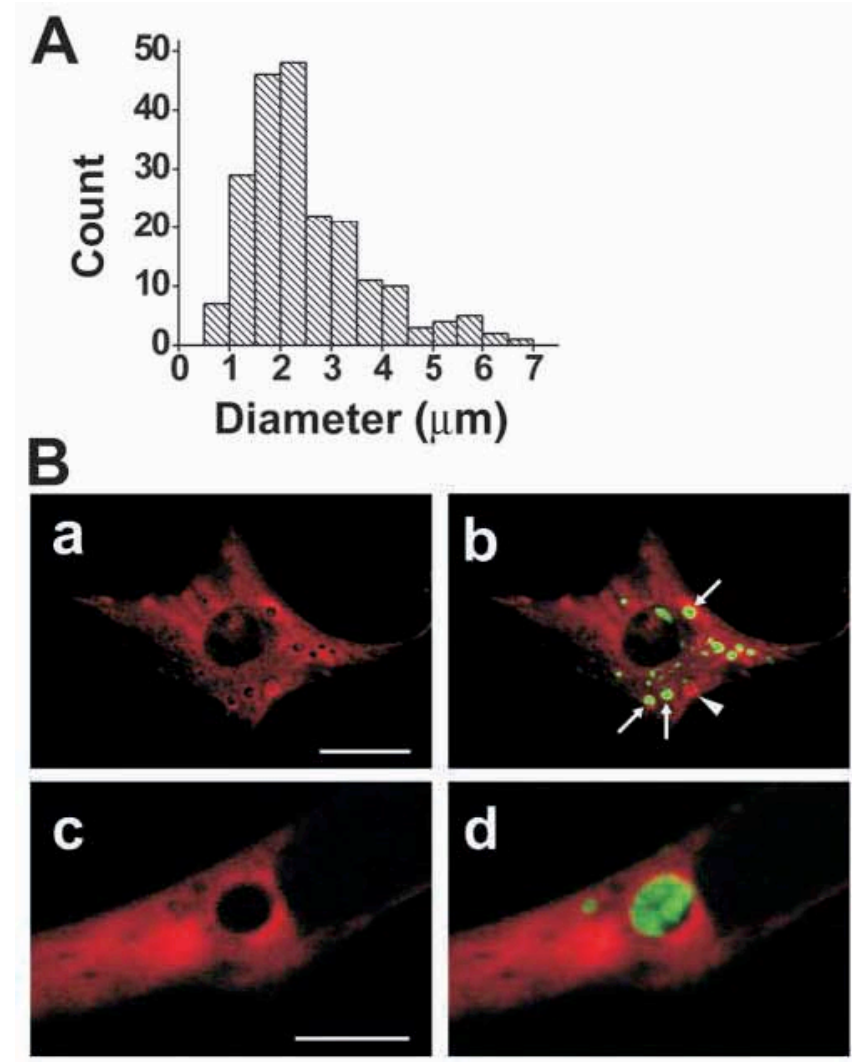

Fig. 8. Characterization of the vesicles labeled by NDP kinase. (A). Size distribution of NDP kinase-labeled vesicles. The histogram was built as described in Materials and Methods. (B) NDP kinase is associated with vesicles labeled by LAMP-1. This image shows the immunolocalization of NDP kinase with Ab-1 (red) and LAMP-1 (green) in quiescent cells. NDP kinase labels the periphery (a) of vesicles, whereas LAMP-1 is visible in the lumen (b, arrows); a circular ruffle (arrowhead) is stained only by NDP kinase. Scale bar, $25 \mu \mathrm{m}$.A large vacuole positive for both NDP kinase (c) and LAMP1 (d) has a multivesicular appearance. Scale bar, $5 \mu \mathrm{m}$.

NDP-kinase-labeled vesicles have many characteristics of the macropinosomes formed by the closing of ruffles (broad size distribution, appearance in phase-contrast optics) (Swanson and Watts, 1995), they lack basic features of these structures. For instance, if NDP kinase were associated with macropinosomes, one would expect to see an increase in the number of labeled vesicles following Rac activation, which stimulates macropinocytosis in murine fibroblasts (Ridley et al., 1992), yet we observed no significant effects of Rac activation on vesicle-associated NDP kinase. More importantly, the structures coated by NDP kinase are not accessible to a fluorescent endocytic tracer (Texas Red dextran) even after an 18 hour chase (not shown), and therefore do not appear to be involved in fluid phase uptake.

The vesicles labeled by NDP kinase are also distinct from structures containing Rab4 (data not shown), a marker of early endosomes (Van der Sluijs et al., 1991). However, double labeling of quiescent NIH-3T3 fibroblasts for NDP kinase and the lysosome-associated membrane protein 1 (LAMP-1) sometimes shows a significant overlap of the two signals in phase-bright vesicles. NDP kinase accumulates around the

\section{Dual localization of NDP kinase 3245}

cytoplasmic surface of the vacuoles, whereas LAMP-1 labels the lumen (Fig. 8Ba,b). Because LAMP-1 is a membrane protein, its apparent localization in the lumen of late endosomes is likely to be a consequence of smaller vesicles being present in the lumen of the larger vacuoles. Indeed, occasionally, the LAMP-1-positive vacuoles labeled by NDP kinase are very large and contain clusters of smaller, round vesicles (Fig. 8Bc,d). The morphological features and strong labeling with anti-LAMP-1 suggests that these structures are multivesicular bodies (MVBs), which are part of the late endosomal compartment and 
participate in the sorting of endocytosed proteins and lipids (Felder et al., 1990). However, late endosomes124 and MVBs should accumulate internalized fluorescent dextran, whereas the vesicles labeled by NDP kinase do not. Thus, the NDP-kinase-positive vesicles, including those with multivesicular appearance, belong to an atypical intracellular compartment that is not active in the classical endocytic pathway.

NDP-kinase-labeled vesicles co-localize with microtubules

MVB-like organelles that are not involved in fluid phase uptake have been identified in neurons through their association with a member of the kinesin superfamily of microtubule motors, KIFC2 (Saito et al., 1997). Given that NDP kinase co-localizes partially with the microtubular network in epithelial cells (Pinon et al., 1999) and the centrosome of C6 glioma cells (Roymans et al., 2001), we investigated the relationship between microtubules and the vesicles coated with NDP kinase by double labeling with antibodies to $\alpha$-tubulin and NDP kinase (Fig. 9). Although treatment with EGF induces pronounced accumulation of NDP kinase in ruffles, microtubules are largely absent from ruffling areas (not shown). However, the signals for the two proteins overlap distinctly around the vesicles labeled by NDP kinase, indicating that the vesicles interact simultaneously with microtubules and NDP kinase. The co-localization of tubulin and NDP kinase at the periphery of vesicles is not a result of serum deprivation because it is also observed in cells cultured in complete medium (Fig. 9). Depolymerization of microtubules with nocodazole eliminates association of tubulin and NDP kinase with large intracellular vesicles (Fig. 9). Thus, clustering of NDP kinase at the periphery of vesicles depends on an intact microtubular network.

NDP kinase is associated with membranes that bind to microtubules in vitro

Our observations are suggestive of an interaction between NDP kinase and microtubule-bound vesicles. To determine whether the overlap in staining reflects a physical association between NDP kinase and microtubule-associated vesicles, we took advantage of a cell-free assay that reproduces the in vivo association between endosomes and microtubules (Goltz et al., 1992; Oda et al., 1995), thus putting the morphological results to a biochemical test. A fraction containing light membranes and cytosol was obtained from NIH-3T3 cells. Endogenous tubulin was polymerized with taxol, and pelleted at $g$ forces sufficient to sediment microtubules and associated structures, but not isolated membranes. The pellet (MT/Ves) was washed extensively and subjected to immunoblot analysis. The MT/Ves fraction contains NDP kinase, tubulin, Rab4 and LAMP-1 (Fig. 10), indicating that both early and late endosomal membranes associate with microtubules under our conditions. Stimulation of cells with EGF does not affect the amount of NDP kinase, tubulin or LAMP-1 in the MT/Ves fraction but eliminates Rab4 from the pellet (Fig. 10). Translocation of Rab4 from endosomes to the cytosol in response to stimulation was previously reported in adipocytes treated with insulin (Cormont et al., 1993) and our data show that EGF has the same effect in fibroblasts. The lack of effect of EGF on the levels of NDP kinase bound to the MT/Ves fraction is consistent with our observations that the association of NDP kinase with vesicles is not altered by extracellular stimulation (Fig. 9) or by expression of activated or dominant-negative Rac (Fig. 5).

NDP kinase co-immunoprecipitates from cell lysates with tubulin (Lombardi et al., 1995; Roymans et al., 2001) and co-localizes partially with microtubules in intact cells (Pinon et al., 1999), although purified NDP kinase does not bind directly to microtubules (Melki et al., 1992). To determine whether the NDP kinase pool bound to the MT/Ves pellet was associated with tubulin prior to the formation of microtubules, the postnuclear supernatant was centrifuged at $230,000 \mathrm{~g}$ to sediment membranes. The supernatant (cytosol) was removed, incubated with taxol to polymerize soluble tubulin and centrifuged at 16,000 $\mathrm{g}$. When both pellets were washed and examined for their NDP kinase content by immunoblotting, we found that NDP kinase is present in the membrane pellet but not in the microtubule pellet (not shown). Thus, the NDP kinase in the MT/Ves pellet derives from membranes, not the tubulin component. This result demonstrates that the association of NDP kinase with the MT/Ves fraction is specific and not due to trapping of this abundant cytosolic protein in the microtubule pellet. 


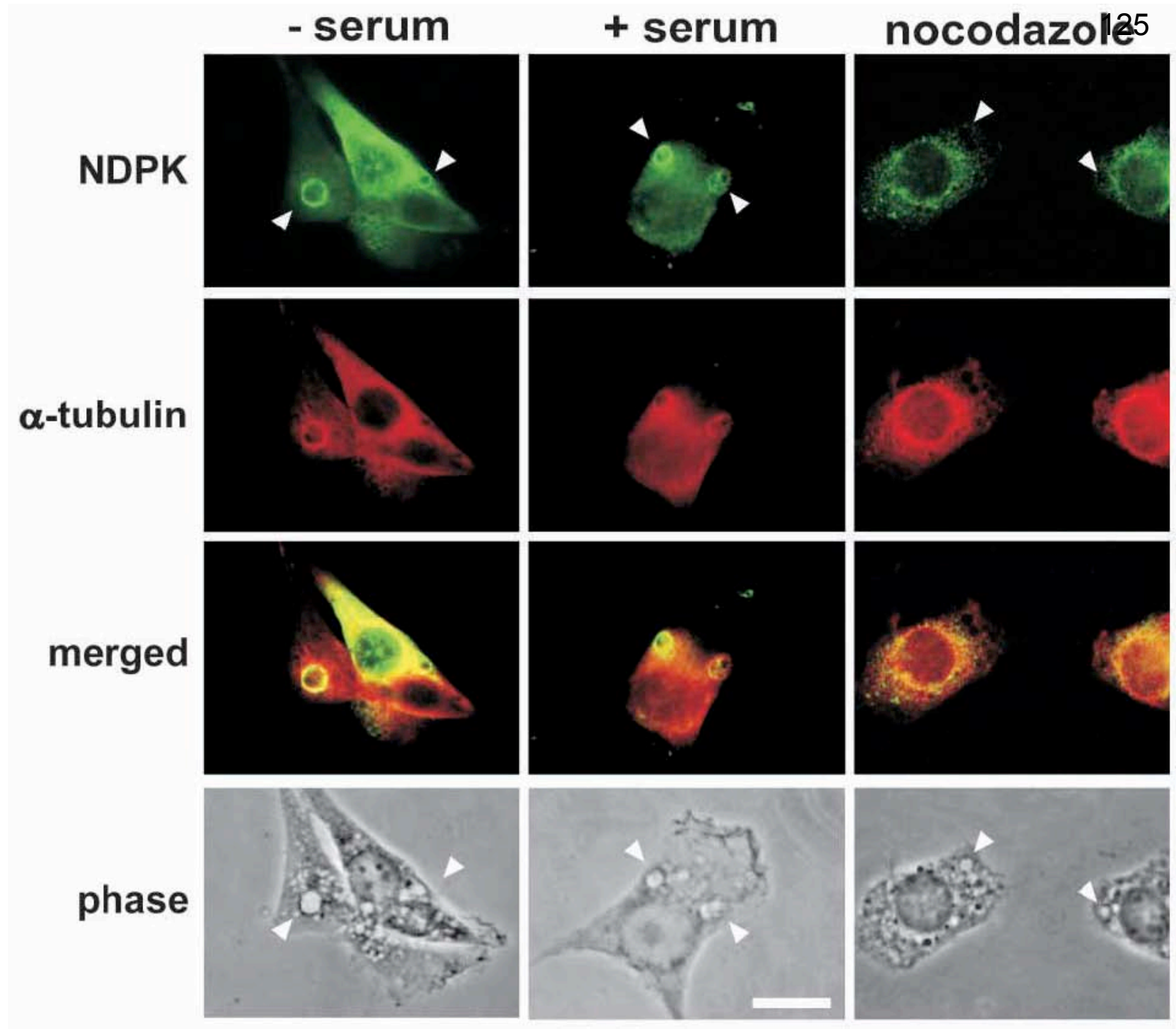

Fig. 9. Microtubular association of NDP kinase-labeled vesicles. Cells were fixed and double stained with antibodies to NDP kinase (Ab-1) and $\alpha$-tubulin after being serum starved for 18 hours (-serum), kept in complete medium (+serum) or treated with $33 \mu \mathrm{M}$ nocodazole for 1 hour (nocodazole). Arrowheads show vesicular structures visible in phase contrast, which are labeled by antibodies to $\alpha$-tubulin and NDP kinase in serum-starved and serum-treated cells (left and middle, respectively). Labeling of vesicles is lost in cells treated with nocodazole (right). Scale bar, $20 \mu \mathrm{m}$.

Fig. 10. NDP kinase pellets with microtubules and endosomal vesicles. Prior to homogenization, cells were incubated in the absence and presence of $10 \mathrm{ng} \mathrm{ml}^{-1}$ EGF for 15 minutes. Taxol-stabilized microtubules and associated membranes were then isolated as described in Materials and Methods, in the absence of nucleotides. Fractions were analysed by immunoblotting with antibodies to NDP kinase, tubulin and the endosomal markers Rab4 and LAMP-1. 
Nucleotides release NDP kinase from microtubule-bound endosomes

Formation of taxol-stabilized microtubules is usually performed in the presence of GTP, even though (at appropriate concentrations) taxol alone is sufficient to induce complete polymerization (Oda et al., 1995). Indeed, inclusion of $1 \mathrm{mM}$ GTP in the incubation with Taxol does not affect the amount of tubulin in the MT/Ves pellet; neither does it affect the association of early and late endosomal membranes with the microtubule pellet, as indicated by the unchanged levels of Rab4 and LAMP-1 (Fig. 11A). By contrast, GTP induces a striking release of NDP kinase from the pellet; ATP also reduces the association of NDP kinase with MT/Ves, but is noticeably and consistently ( $n=6$ ) less effective than GTP (Fig. 11A). Thus, NDP kinase is released from MT/Ves through a nucleotide-sensitive site that shows some specificity for the base.

The nucleotide-induced release of NDP kinase from the MT/Ves fraction qualitatively resembles the behavior of the molecular motors dynein and kinesin, whose binding to microtubules and associated endosomal vesicles is also responsive to nucleotides (Oda et al., 1995). However, we find that the amounts of dynein and kinesin associated with the MT/Ves fraction are not altered by $1 \mathrm{mM}$ GTP or ATP (not shown). Presumably the interaction of NDP kinase with MT/Ves is more sensitive to nucleotides than that of molecular motors, so the nucleotide concentration used in this work is too low to cause elution of significant amounts of dynein and kinesin. For instance, $10 \mathrm{mM}$ ATP releases only $50 \%$ of bound dynein and kinesin from an analogous fraction obtained from rat liver (Oda et al., 1995), with GTP being slightly less effective. Thus, the dissociation of NDP kinase from the MT/Ves pellet by nucleotides is not linked to the release of motor proteins and their cargo.

NDP kinases bind nucleotides with affinities in the range of 10-200 $\mu \mathrm{M}$, and guanine nucleotides are somewhat preferred over other substrates (Schaertl et al., 1998; Cervoni et al., 2001; Schneider et al., 2002). Therefore, the simplest explanation for the preferential release of NDP kinase from the MT/Ves fraction by GTP is that it involves binding of the nucleotide directly to its catalytic site. However, it is also possible that the release of NDP kinase from the MT/Ves fraction is secondary to the interaction of GTP with a highly selective binding site, perhaps a GTP-binding protein, and that the effect of ATP is indirect, requiring its conversion into GTP. To distinguish 


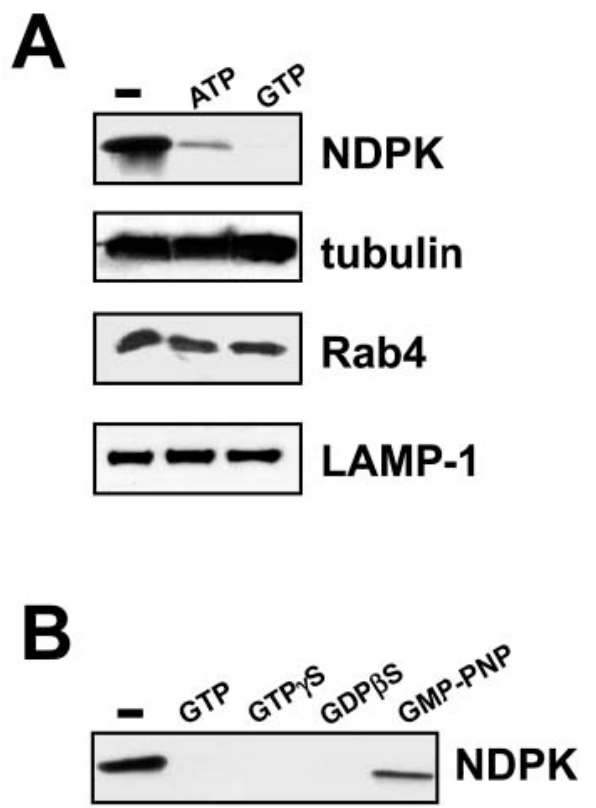

Fig. 11. Nucleotides release NDP kinase from taxol-stabilized microtubules and associated vesicles. (A) An extract from resting cells was divided into equal portions that were incubated with Taxol in buffer supplemented or not with ATP or GTP (both at $1 \mathrm{mM}$ ); after washes, pellets were tested for NDP kinase, tubulin, Rab4 and LAMP-1. (B) As above; binding of NDP kinase to the MT/Ves pellets was assessed in samples where GTP or guanine nucleotide analogs were included in the incubation with taxol.

between these possibilities, we examined the effects of the guanine nucleotide analogs guanosine-5'-O-(3-thio)triphosphate (GTP $\gamma \mathrm{S})$, guanylyl-imidodiphosphate (GMPPNP) and guanosine-5'-O-(2-thio)diphosphate (GDP $\beta \mathrm{S}$ ) on the association of NDP kinase with the MT/Ves fraction. If release of NDP kinase by GTP is related to a GTP-binding protein, the activating GTP analogs GTP $\gamma \mathrm{S}$ and GMP-PNP are expected not only to act alike but also to have opposite effects to those of GDP $\beta$ S, which locks GTP-binding proteins in the inactive form. However, if the release is mediated by the catalytic site of NDP kinase, the thiophosphate analogs GTP $\gamma \mathrm{S}$ and GDP $\beta \mathrm{S}$, which are substrates for NDP kinases (Schaertl et al., 1998), should behave similarly to GTP. Imidodiphosphate analogs bind to NDP kinases with low affinity and are not substrates (Cervoni et al., 2001), so GMP-PNP should have modest effects, if any, on the retention of NDP kinase by the MT/Ves fraction. As seen in Fig. 11B, $1 \mathrm{mM} \mathrm{GTP \gamma} \mathrm{S}$ and GDP $\beta$ S, but not GMP-PNP, are as effective as GTP in eluting NDP kinase, suggesting that the nucleotide binding site involved is that of NDP kinase.

\section{Discussion}

To investigate further the role of NDP kinase in transmembrane signaling, we sought to identify subcellular compartments in which it is present in resting cells and to determine whether activation of surface receptors can cause rapid changes in the pattern of NDP kinase localization. We found that, in NIH-3T3 cells, there are two discernible cytoplasmic pools of NDP kinase whose location is regulated independently by distinct cellular signals: a proportion of the cellular NDP kinase is rapidly translocated to lamellipodia and ruffles in response to activation of RTKs and GPCRs, whereas another NDP kinase pool is associated with cytoplasmic vesicles in both quiescent and stimulated cells. Comparison with the distribution of the cytosolic protein GFP shows that the intense staining seen around vesicles and in ruffles is not a volume effect but reflects real accumulation of NDP kinase. The clear differences between cortical and vesicular NDP kinase pools in terms of localization and response to extracellular stimulation suggest strongly that they use different targeting devices. 
Although we used two different antibodies as tools, a monoclonal specific to NDP kinase A and a128 polyclonal that recognizes both NDP kinase A and B both highlight the same structures. This is not surprising, given that the bulk of mammalian NDP kinases A and B are present in cells as stable mixed hexamers (Gilles et al., 1991). This finding is also consistent with a report that isoforms A and B are similarly distributed in the cytoplasmic space under normal culture conditions (Pinon et al., 1999); nevertheless, the possibility that the ratio of one isoform to another might change substantially as a function of the compartment examined (Barraud et al., 2002) cannot be overlooked. Another caveat is that the antibodies used here were tested against NDP kinase A and B, which are by far the most abundant isoforms in mammals (Lacombe et al., 2000). Given that some NDP kinase proteins are yet to be isolated, it is possible (although unlikely) that our results reflect the localization of cross-reacting NDP kinase isoforms that are expressed at low levels and undetectable by immunoblotting but might be concentrated in specific cell locations; a definite answer will have to await the purification of all NDP kinase isoforms and development of high-affinity isoform-specific tools.

Translocation of molecules into ruffles is often achieved by binding to proteins enriched in these structures, such as F-actin or activated Rac1. However, NDP kinase does not bind to F-actin or Rac in vitro. NDP kinase also does not carry consensus sequences found in Rac effectors, Rac GEFs or in Rac GTPase activating proteins. Therefore, its connection to Rac activation is probably indirect. Otsuki et al. reported that NDP kinase A associates with the Rac1 nucleotide exchange factor Tiam1 and that overexpression of NDP kinase A inhibits the activating effect of Tiam1 on Rac (Otsuki et al., 2001). However, our experiments show that, in serum-starved NIH-3T3 cells stimulated briefly with EGF, ruffles that are strongly stained for NDP kinase contain little Tiam1, suggesting that association with Tiam1 does not mediate translocation of NDP kinase A upon Rac activation. Recently, Palacios et al. demonstrated that, in MDCK epithelial cells, the small GTPase ARF6 mediates the recruitment of heterologously expressed human NDP kinase A to areas of cell-cell contact (Palacios et al., 2002). Once translocated to these sites, NDP kinase A would facilitate dynamin-dependent endocytosis and downregulate Rac activation by binding Tiam 1 . We are currently investigating whether an analogous interaction with ARF6 can mediate the rapid translocation of endogenous NDP kinase to the cortex of stimulated NIH-3T3 fibroblasts.

NDP kinase B was also found to be directed to ruffles, through interaction with ICAP1- $\alpha$ (Fournier et al., 2002). However, we use adherent cells and ICAP1- $\alpha$ reportedly affects cell morphology and behavior only during cell adhesion (Degani et al., 2002), so a similar mechanism is not likely to be responsible for the rapid translocation of NDP kinase in response to EGF and bombesin reported here.

The marked effect of BDM on NDP kinase localization (Fig. 4) suggests yet another alternative, namely that a member of the myosin family might be involved. However, because BDM is also known to affect calcium homeostasis and microtubule function (Kiehart, 1999), the precise mechanism of its action requires careful investigation. Despite the uncertainty as to the underlying mechanism, the demonstration that a proportion of cellular NDP kinases is quickly redistributed following cell stimulation and Rac activation strongly supports previous data indicating that these proteins are involved in signaling (reviewed in Otero, 2000).

The finding that, in NIH-3T3 cells, NDP kinase localizes constitutively to the surface of intracellular vesicles was surprising. Based on their inaccessibility to a fluid phase uptake marker, we conclude that these structures are not macropinosomes. Some of the structures ringed by NDP kinase in quiescent cells contain LAMP-1, a marker of late endosomes/lysosomes; occasionally, the NDP kinase/LAMP-1-positive vesicles resemble multivesicular bodies, which are part of the late endosomal compartment. However, double labeling with markers for endocytic compartments indicates that the vesicles labeled by NDP kinase antibodies are distinct from early and recycling endosomes, and lack fundamental features of late endosomes and MVBs. Thus, most of the NDP-kinase-positive vesicles are not conventional endocytic organelles and might be part of the secretory pathway. Although the analysis of the protein composition of the vesicles coated by NDP kinase is currently being performed, the possibility of a link between NDP kinase and secretory vesicles is backed by recent work showing that, in neurons, NDP kinases localize to membranes from the Golgi and endoplasmic reticulum, and to vesicles budding from the trans-Golgi (Barraud et al., 2002).

The morphological evidence presented here points to an interaction between NDP kinase and discrete vesicles that associate with microtubules. The co-localization of NDP kinase and tubulin seen by immunofluorescence is not coincidental: a separate experimental approach using a cell-free assay 
demonstrates that the overlap reflects a physical association between NDP kinase, stabilized microtubules 129 and membrane vesicles. The tight binding of NDP kinase to this fraction and the full release induced by GTP and other substrates suggest that the interaction is specific. We hypothesize that the dynamic association of NDP kinase with microtubule-bound vesicles in fibroblasts is relevant to the operation of the multiple GTPases that control intracellular membrane transport. Namely, in vivo, NDP kinase might associate with microtubule-bound vesicles when GTP levels are low, using nucleoside triphosphates such as ATP to phosphorylate GDP, whereas a rise in GTP levels leads to its release. Our data agree with studies by other groups implicating NDP kinase in intracellular vesicle trafficking processes. Thus, NDP kinase B is a component of isolated phagosomes (Garin et al., 2001) and the Drosophila homolog of NDP kinase, Awd, regulates dynamin-dependent synaptic vesicle recycling through a mechanism that requires its intrinsic NDP kinase activity, presumably GTP regeneration (Krishnan et al., 2001). Facilitation of dynamin-based endocytosis by NM23-H1 was also reported for mammalian cells (Palacios et al., 2002). Recently, Baillat et al. (Baillat et al., 2002) demonstrated a direct interaction between NDP kinase and dynamin I, as well NDP kinase and phocein, which is homologous to the $\sigma$ subunits of clathrin adaptor subunits, supporting the idea that NDP kinase plays a role in vesicular traffic.

We are grateful to A. V. Somlyo and L. Geddis for materials, advice and helpful discussions. We thank I. Lascu for the kind gift of the antibody to NDP kinase used in immunoblots. Monoclonal antibodies developed by T. August (1D4B), J. Scholey (SUK4) and D. Fambrough ( $\alpha$ 5) were obtained from the Developmental Studies Hybridoma Bank developed under the auspices of the NICHD and maintained by The University of Iowa, Department of Biological Sciences (Iowa City, IA 52242). We are supported by DHHS grants GM57833 to AO and GM62762 to GS.

\section{References}

Baillat, G., Gaillard, S., Castets, F. and Monneron, A. (2002). Interactions of phocein with nucleoside-diphosphate kinase, Eps15 and dynamin I. J. Biol. Chem. 277, 18961-18966.

Barraud, P., Amrein, L., Dobremez, E., Dabernat, S., Masse, K., Larou, M., Daniel, J. Y. and Landry, M. (2002). Differential expression of $n m 23$ genes in adult mouse dorsal root ganglia. J. Comp. Neurol. 444, 306-323.

Bourguignon, L. Y. W., Zhu, H., Shao, L. and Chen, Y. W. (2000). AnkyrinTiam1 interaction promotes Rac1 signaling and metastatic breast tumor cell invasion and migration. J. Cell Biol. 150, 177-191.

Cervoni, L., Lascu, I., Xu, Y., Gonin, P., Morr, M., Merouani, M., Janin, J. and Giartosio, A. (2001). Binding of nucleotides to nucleoside diphosphate kinase: a calorimetric study. Biochemistry 40 , 4583-4589.

Cormont, M., Tanti, J. F., Zahraoui, A., van Obberghen, E., Tavitian, A. and le Marchand-Brustel, Y. (1993). Insulin and okadaic acid induce Rab4 redistribution in adipocytes. J. Biol. Chem. 268, 19491-19497.

Cramer, L. P. and Mitchison, T. J. (1995). Myosin is involved in postmitotic cell spreading. J. Cell Biol. 131, 179-189.

Degani, S., Balzac, F., Brancaccio, M., Guazzone, S., Retta, S. F., Silengo, L., Eva, A. and Tarone, G. (2002). The integrin cytoplasmic domain-associated protein ICAP-1 binds and regulates Rho family GTPases during cell spreading. J. Cell Biol. 156, 377-387.

Del Pozo, M. A., Kiosses, W. B., Alderson, N. B., Meller, N., Hahn, K.

M. and Schwartz, M. A. (2002). Integrins regulate GTP-Rac localized effector interactions through dissociation of Rho-GDI. Nat. Cell Biol. 4, 232-239.

Felder, S., Miller, K., Moehren, G., Ullrich, A., Schlessinger, J. and Hopkins, C. R. (1990). Kinase activity controls the sorting of the epidermal growth factor receptor within the multivesicular body. Cell 61, 623-634.

Fournier, H. N., Dupe-Manet, S., Bouvard, D., Lacombe, M. L., Marie, C., Block, M. R. and Albiges-Rizo, C. (2002). ICAP-1alpha interacts directly with the metastasis suppressor $\mathrm{nm} 23-\mathrm{H} 2$ and both proteins are targeted to newly formed cell adhesion sites upon integrin engagement. J. Biol. Chem. 277, 20895-20902.

Garin, J., Diez, R., Kieffer, S., Dermine, J. F., Duclos, S., Gagnon, E., Sadoul, R., Rondeau, C. and Desjardins, M. (2001). The phagosome proteome: insight into phagosome functions. J. Cell Biol. 152, 165-180.

Gilles, A. M., Presecan, E., Vonica, A. and Lascu, I. (1991). Nucleoside diphosphate kinase from human erythrocytes. Structural characterization of the two polypeptide chains responsible for heterogeneity of the hexameric enzyme. J. Biol. Chem. 266, 8784-8789.

Goltz, J. S., Wolkoff, A. W., Novikoff, P. M., Stockert, R. J. and Satir, P.

(1992). A role for microtubules in sorting endocytic vesicles in rat hepatocytes. Proc. Natl. Acad. Sci. USA 89, 7026-7030.

Kaksonen, M., Peng, H. B. and Rauvala, H. (2000). Association of cortactin with dynamic actin in lamellipodia and on endosomal vesicles. J. Cell Sci. 113, 4421-4426.

Kantor, J. D., McCormick, B., Steeg, P. S. and Zetter, B. R. (1993). Inhibition of cell motility after NDP kinase transfection of human and murine tumor cells. Cancer Res. 53, 1971-1973.

Dual localization of NDP kinase 3249

Kierhart, D. P. (1999). Wound healing: the power of the purse string. Curr. Biol. 9, R602-R605. 
Kimura, N., Shimada, N., Fukuda, M., Ishijima, Y., Miyazaki, H., Ishii, A., Takagi, Y. and Ishikawa, N. (2000). Regulation of 130 cellular functions by nucleoside diphosphate kinases in mammals. J. Bioenerg. Biomembr. 32, 309-315.

Kjöller, L. and Hall, A. (1999). Signaling to Rho GTPases. Exp. Cell Res. 253, 166-179.

Kraeft, S., Traincard, F., Bourdais, J., Mesnildrey, S., Veron, M. and Chen,

L. B. (1996). Nuclear localization of nucleoside diphosphate kinase type B (nm23-H2) in cultured cells. Exp. Cell Res. 227, 63-69.

Krishnan, K. S., Rikhy, R., Rao, S., Shivalkar, M., Mosko, M., Narayanan, R., Etter, P., Estes, P. S. and Ramaswami, M. (2001). Nucleoside diphosphate kinase, a source of GTP, is required for dynamin-dependent synaptic vesicle recycling. Neuron 30, 197-210.

Lacombe, M. L., Milon, L., Munier, A., Mehus, J. G. and Lambeth, D. O.

(2000). The human Nm23/nucleoside diphosphate kinases. J. Bioenerg. Biomembr. 32, 247-258.

Lombardi, D., Sacchi, A., D’Agostino, G. and Tibursi, G. (1995). The association of the Nm23-M1 protein and beta-tubulin correlates with cell differentiation. Exp. Cell Res. 217, 267-271.

Melki, R., Lascu, I., Carlier, M. F. and Veron, M. (1992). Nucleoside diphosphate kinase does not directly interact with tubulin nor microtubules. Biochem. Biophys. Res. Commun. 187, 65-72.

Michiels, F., Stam, J. C., Hordijk, P. L., van der Kammen, R. A., Ruuls-Van Stalle, L., Feltkamp, C. A. and Collard, J. G. (1997). Regulated membrane localization of Tiam1, mediated by the NH2-terminal pleckstrin homology domain, is required for Rac-dependent membrane ruffling and c-Jun NH2-terminal kinase activation. J. Cell Biol. 137, 387-398.

Oda, H., Stockert, R. J., Collins, C., Wang, H., Novikoff, P. M., Satir, P. and Wolkoff, A. W. (1995). Interaction of the microtubule cytoskeleton with endocytic vesicles and cytoplasmic dynein in cultured rat hepatocytes. J. Biol. Chem.270, 15242-15249.

Otero, A. S. (1997). Copurification of vimentin, energy metabolism enzymes, and a MER5 homolog with nucleoside diphosphate kinase. Identification of tissue-specific interactions. J. Biol. Chem. 272, 14690-14694.

Otero, A. S. (2000). NM23/nucleoside diphosphate kinase and signal transduction. J. Bioenerg. Biomembr. 32, 269-276.

Otero, A. S., Doyle, M. B., Hartsough, M. T. and Steeg, P. S. (1999). Wild-type NM23-H1, but not its S120 mutants, suppresses desensitization of muscarinic potassium current. Biochim. Biophys. Acta 1449, 157-168.

Otsuki, Y., Tanaka, M., Yoshii, S., Kawazoe, N., Nakaya, K. and Sugimura, H. (2001). Tumor metastasis suppressor nm23H1 regulates Rac1 GTPase

by interaction with Tiam1. Proc. Natl. Acad.Sci. USA 98, 4385-4390. Palacios, F., Schweitzer, J. K., Boshans, R. L. and

D'Souza-Schorey, C.

(2002). Arf6-GTP recruits NM23-H1 to facilitate dynamin-mediated endocytosis during adherens junctions disassembly. Nat. Cell Biol. 4, 929936.

Pinon, V. P. B., Millot, G., Munier, A., Vassy, J., Linares-Cruz, G., Capeau, J., Calvo, F. and Lacombe, M. L. (1999). Cytoskeletal association of the A and B nucleoside diphosphate kinases of interphasic but not mitotic human carcinoma cell lines: specific nuclear localization of the B subunit. Exp. Cell Res. 246, 355-367.

Postel, E. H., Abramczyk, B. A., Gursky, S. K. and Xu, Y. (2002). Structure-based mutational and functional analysis identify human NM23-H2 as a multifunctional enzyme. Biochemistry 41, 6330-6337.

Ridley, A. J., Paterson, H. F., Johnston, C. L., Diekmann, D. and Hall, A. (1992). The small GTP-binding protein Rac regulates growth factor-induced membrane ruffling. Cell 70, 401-410.

Rottner, K., Hall, A. and Small, J. V. (1999). Interplay between Rac and Rho in the control of substrate contact dynamics. Curr. Biol. 9, 640-648.

Roymans, D., Vissenberg, K., de Jonghe, C., Willems, R., Engler, G., Kimura, N., Grobben, B., Claes, P., Verbelen, J. P., van Broeckhoven,

C. et al. (2001). Identification of the tumor metastasis suppressor Nm23-H1/Nm23-R1 as a constituent of the centrosome. Exp. Cell Res. 262, 145153

Saito, N., Okada, Y., Noda, Y., Kinoshita, Y., Kondo, S. and Hirokawa, N. (1997). KIFC2 is a novel neuron-specific C-terminal type kinesin superfamily motor for dendritic transport of multivesicular body-like organelles. Neuron 18, $425-438$.

Schaertl, S., Konrad, M. and Geeves, M. A. (1998). Substrate specificity of human nucleoside-diphosphate kinase revealed by transient kinetic analysis.

J. Biol. Chem.273, 5662-5669. Schneider, B., Norda, A., Karlsson, A., Veron, M. and Deville-Bonne, D.

(2002). Nucleotide affinity for a stable phosphorylated intermediate of nucleoside diphosphate kinase. Protein Sci. 11, 1648-1656.

Swanson, J. A. and Watts, C. (1995). Macropinocytosis. Trends Cell Biol. 5, 424-428.

Van der Sluijs, P., Hull, M., Zahraoui, A., Tavitian, A., Goud, B. and

Mellman, I. (1991). The small GTP-binding protein Rab4 is associated with early endosomes. Proc. Natl. Acad. Sci. USA 88, 6313-6317.

Xu, L., Murphy, J. M. and Otero, A.S. (1996). Participation of nucleoside-diphosphate kinase in muscarinic K ${ }^{+}$channel activation does not involve GTP formation. J. Biol. Chem. 271, 21120-21125. 